\title{
KAZHDAN-LUSZTIG EQUIVALENCE AND FUSION OF KAC MODULES IN VIRASORO LOGARITHMIC MODELS
}

\author{
P.V. BUSHLANOV, A.M. GAINUTDINOV AND I.YU. TIPUNIN
}

\begin{abstract}
The subject of our study is the Kazhdan-Lusztig (KL) equivalence in the context of a one-parameter family of logarithmic CFTs based on Virasoro symmetry with the $(1, p)$ central charge. All finite-dimensional indecomposable modules of the KL-dual quantum group - the "full" Lusztig quantum $s \ell(2)$ at the root of unity - are explicitly described. These are exhausted by projective modules and four series of modules that have a functorial correspondence with any quotient or a submodule of Feigin-Fuchs modules over the Virasoro algebra. Our main result includes calculation of tensor products of any pair of the indecomposable modules. Based on the Kazhdan-Lusztig equivalence between quantum groups and vertex-operator algebras, fusion rules of Kac modules over the Virasoro algebra in the $(1, p)$ LCFT models are conjectured.
\end{abstract}

\section{INTRODUCTION}

Logarithmic conformal field theories (LCFTs) have proven to be one of the richest subjects in theoretical and mathematical physics with applications in a "non-unitary" world ranging from modeling avalanche processes [1], observables in stochastic processes $\operatorname{SLE}(\kappa, \rho)$ [2], and surface critical behaviour in $\mathrm{O}(n)$ models and loop models [3], to percolation probabilities [4, 5, 6], and edge states in the quantum Hall effect [7, 8]. Beside the physical applications, LCFTs give rise to subjects of intense studies from a more formal point of view including a free-field representation [9, 10, 11, 12], vertex-operator algebras approach [13, 14], Zhu algebras aspects and super W-algebras [15], quantum-group dualities [16, 17, 18] and Verlinde algebras [11, 19, 20, 21], construction of a new class of W-algebras extending symmetry in rational CFTs based on affine $s \ell(2)$ [22], an interplay between rational boundary LCFTs and non-semisimple braided finite tensor categories [23, 24], and recently in defining a wide family of LCFTs parametrized by Dynkin diagrams [25].

One of important achievements made in studying LCFTs has been the systematic definition of chiral algebras in terms of so-called screening currents [11, 12]. The idea is to use screening operators intertwining a Virasoro-module structure on a lattice VOA, and to define chiral algebras relevant for LCFTs as the kernel of the screening operators. By contrast, chiral algebras defining RCFTs are usually defined as cohomologies of the screening operators [26]. Subsequently, the "screening kernel" approach in defining LCFTs has led to explicit construction of quantum groups (at roots of unity) centralizing the chiral algebras [12, 27]. Such quantum-group symmetry in the space of states allowed to describe representation categories 
of the chiral algebras [16, 17, 27] - a correspondence between subquotient structures of modules over the chiral algebra and over its centralizing quantum group, and between their fusion rules was stated1. In a simplest LCFT such as symplectic fermions [28] an equivalence on the level of braided tensor categories and modular group action was proven in [29]. Such nice correspondences, in a representation-theoretic (or functorial) sense, give an extended analogue of the so-called Kazhdan-Lusztig equivalence established first in a context of affine Lie algebras at negative integer levels [30].

The Kazhdan-Lusztig (KL) equivalence was well tested so far in several cases of rational LCFTs [16, 17], with a recent contribution in [31]. In particular, a KL equivalence between a restricted (or "small") quantum $s \ell(2)$ at the primitive $2 p$ th root of unity (based on a "short" screening and denoted by $\left.\overline{\mathcal{U}}_{\mathfrak{q}} s \ell(2)\right)$ and the triplet $W$-algebra [32] realizing an extended conformal symmetry in $(1, p)$ logarithmic conformal field models was established in [16] while a proof of this equivalence on the level of abelian representation categories was given quite recently [33].

Another achievement in defining and studying logarithmic theories was proposed few years ago [34, 35, 6], based on a construction of lattice discretizations of the LCFTs. The point is that it is still difficult to compute fusion rules and determine subquotient structure of indecomposable representations of the chiral algebra that should appear in continuum logarithmic theories. Lattice discretizations, on the other hand, naturally involve well-studied "lattice" algebras such as Temperley-Lieb (TL) [36, 37, 38], Brauer [39, 40], different types of blob algebras [41, 42, 3] and their centralizing quantum groups [43, 44] as well. The transfer matrix and the Hamiltonian operators are particular elements of these "lattice" algebras and much intuition as well as rigorous results can be obtained from the study of these lattice features. In particular, the blob (or boundary TL) algebras give a quick access to a description of (integrable) boundary conditions which carry over rather straightforwardly to Virasoro-symmetric boundary conditions in the continuum limit [6, 35, 3] giving thus examples of non-rational LCFTs which involve infinitely many primary fields and their logarithmic partners.

The purpose of this paper is to accomplish an important step forward in the study of nonrational LCFTs by using the KL equivalence. The subject of our study is a one-parameter family of chiral logarithmic models with the $(1, p)$ central charge $c_{1, p}=13-6 / p-6 p$, with integer $p \geqslant 2$. These models were originally formulated as the continuum limit of XXZ spinchains at appropriate roots of unity [35] and as the limit of integrable lattice face models [6]. Both are based on the TL algebra which morally gives a regularization of the stress-energy tensor modes (Virasoro generators) on a finite system [46]. The chiral algebra for these models in the continuum is (a representation of) the Virasoro algebra of the central charge $c_{1, p}$. We will denote these LCFTs as $\mathcal{L} \mathcal{M}(1, p)$, following notations in [6]. Fusion rules in

\footnotetext{
${ }^{1}$ In the context of $\left(p, p^{\prime}\right)$ models, a one-to-one correspondence, i.e. an equivalence, was stated only in the case $p^{\prime}=1$ while for coprime $p, p^{\prime} \neq 1$ the correspondence was stated up to minimal models contribution.
} 
these conformal models were originally studied using an implementation of the GaberdielKausch-Nahm (GKN) algorithm [47] and then investigated in a more systematic way in [35] and in [48, 49], combining lattice computations with the GKN algorithm.

We conjectured in [27] that a KL-type equivalence exists between a "long" screening extension of $\overline{\mathcal{U}}_{\mathfrak{q}} s \ell(2)$ - the Lusztig limit $\mathcal{L} \mathcal{U}_{\mathfrak{q}} s \ell(2)$ of the full quantum $s \ell(2)$ as $\mathfrak{q} \rightarrow e^{\imath \pi / p}-$ and the Virasoro vertex-operator algebra $\mathcal{V}_{p}$ defined by the $S L(2)$-invariant subspace in the vacuum module of the triplet $W$-algebra. Moreover, the fact that the TL algebra and a representation of the quantum group $\mathcal{L} \mathcal{U}_{\mathfrak{q}} s \ell(2)$ centralize each other in the $\mathrm{XXZ}$ spin chains [45, 43, 35] suggests that in the continuum limit many results about $\mathcal{L} \mathcal{M}(1, p)$ can be reformulated in terms of $\mathcal{L} \mathcal{U}_{\mathfrak{q}} s \ell(2)$ too. For existence of the KL equivalence in the context of LCFTs with the Virasoro chiral algebra, there are pieces of evidence which we bring in series.

In [27], the tensor products of all irreducible and projective modules over $\mathcal{L} \mathcal{U}_{\mathfrak{q}} s \ell(2)$ were calculated and identified with the fusion of irreducible and logarithmic (staggered) modules over $\mathcal{V}_{p}$. In this paper, we give an exhaustive description of all indecomposable modules in the category of finite dimensional $\mathcal{L} \mathcal{U}_{\mathfrak{q}} s \ell(2)$-modules and calculate all their tensor products. This allows us to identify indecomposable $\mathcal{L} \mathcal{U}_{\mathfrak{q}} s \ell(2)$-modules with indecomposable Kac modules 2 over $\mathcal{V}_{p}$, by comparison between their subquotient structure, i.e. on the level of abelian categories, using our results from [27]. Then, we conjecture fusion rules for the Kac modules using tensor product decompositions for the corresponding modules over $\mathcal{L} \mathcal{U}_{\mathfrak{q}} s \ell(2)$. Our results agree with ones in [49].

1.1. Results. In order to describe results of this paper, we use quite basic terminology in category theory [51]. Our results consist in the following. Let $\mathcal{C}_{p}$ denotes the category of finite dimensional $\mathcal{L} \mathcal{U}_{\mathfrak{q}} s \ell(2)$-modules. Then, $\mathcal{C}_{p}$ is a direct sum of two full subcategories $\mathcal{C}_{p}=\mathcal{C}_{p}^{+} \oplus \mathcal{C}_{p}^{-}$such that there are no morphisms between $\mathcal{C}_{p}^{+}$and $\mathcal{C}_{p}^{-}$and the subcategory $\mathcal{C}_{p}^{+}$ is closed under tensor products. Categories $\mathcal{C}_{p}^{+}$and $\mathcal{C}_{p}^{-}$are equivalent as abelian categories. We let $\nu: \mathcal{C}_{p}^{+} \rightarrow \mathcal{C}_{p}^{-}$denotes an equivalence functor. The tensor product of any two objects in $\mathcal{C}_{p}^{-}$belongs to $\mathcal{C}_{p}^{+}$and tensor product of an object from $\mathcal{C}_{p}^{+}$with an object from $\mathcal{C}_{p}^{-}$belongs to $\mathcal{C}_{p}^{-}$. This determines a $\mathbb{Z}_{2}$ structure on the tensor category $\mathcal{C}_{p}$. To calculate tensor product for any pair of objects in $\mathcal{C}_{p}$, it is enough to know tensor products in $\mathcal{C}_{p}^{+}$. Let $Y$ be an object from $\mathrm{C}_{p}^{+}$and $Y^{\prime}, Y^{\prime \prime}$ are objects from $\mathcal{C}_{p}^{-}$. Then, $Y \otimes Y^{\prime}=\nu\left(Y \otimes \nu^{-1}\left(Y^{\prime}\right)\right)$ and $Y^{\prime} \otimes Y^{\prime \prime}=\nu^{-1}\left(Y^{\prime}\right) \otimes \nu^{-1}\left(Y^{\prime \prime}\right)$. Therefore, we describe only structure of $\mathcal{C}_{p}^{+}$in detail.

The set of indecomposable modules in the category $\mathcal{C}_{p}^{+}$consists of irreducible modules $\mathrm{X}_{s, r}$, for any pair of integers $1 \leqslant s \leqslant p$ and $r \geqslant 1$, their projective covers $\mathrm{P}_{s, r}$ (which are simultaneously projective and injective objects in $\mathrm{C}_{p}^{+}$) and modules $\mathrm{N}_{s, r}(n), \boldsymbol{U}_{s, r}(n), \mathrm{M}_{s, r}(n)$ and $\mathrm{W}_{s, r}(n)$, with $1 \leqslant s \leqslant p-1$ and $r, n \geqslant 1$. To describe briefly the irreducible module $\mathrm{X}_{s, r}$, we note that it is a tensor product of $s$-dimensional irreducible $\overline{\mathcal{U}}_{\mathfrak{q}} s \ell(2)$-module and

\footnotetext{
${ }^{2} \mathrm{By}$ a Kac module associated with any pair of integers $(r, s), r, s \geqslant 1$, we call the quotient of the corresponding Feigin-Fuchs module [50] by a singular vector on the level $r s$, see a precise definition below.
} 
$r$-dimensional irreducible $s \ell(2)$-module (see precise definitions in Sec. 2.3 ). The projective cover $\mathrm{P}_{s, r}$ of $\mathrm{X}_{s, r}$ has the following subquotient structure:
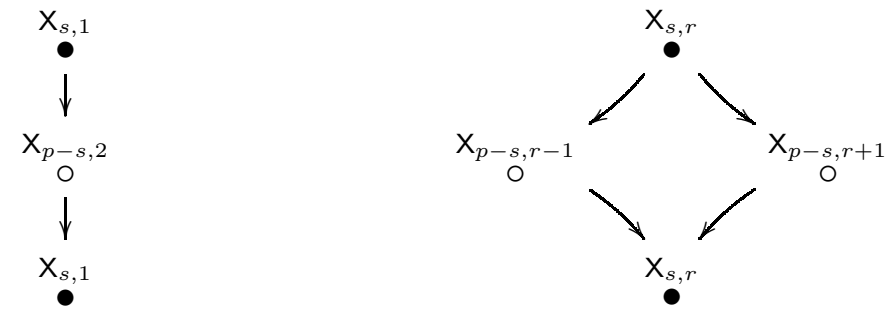

where $r \geqslant 2$ and $1 \leqslant s \leqslant p-1$. We note also that $\mathrm{P}_{p, r}=\mathrm{X}_{p, r}$. The set of irreducible and projective modules is closed under tensor products.

All other indecomposable objects in $\mathrm{C}_{p}^{+}$have subquotient structures in the following list (see also Thm 3.4), where we set $1 \leqslant s \leqslant p-1$ and $r, n \geqslant 1$.

$\mathrm{W}_{s, r}(n)$ :

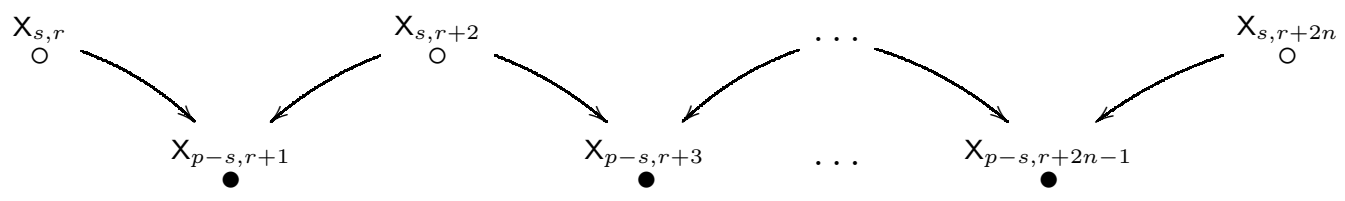

$\mathrm{M}_{s, r}(n)$ :

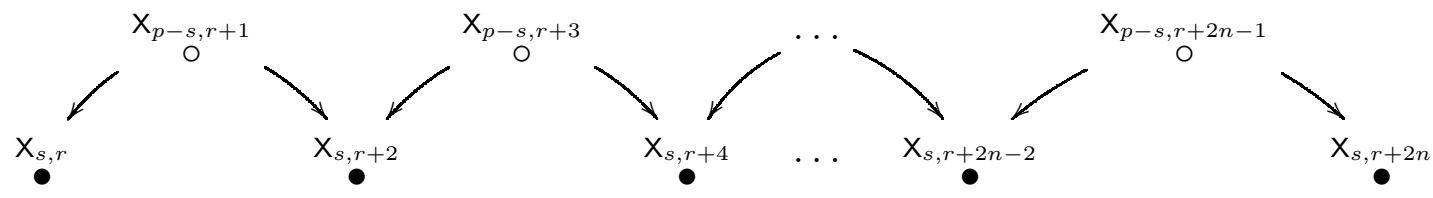

$\mathrm{N}_{s, r}(n)$ :

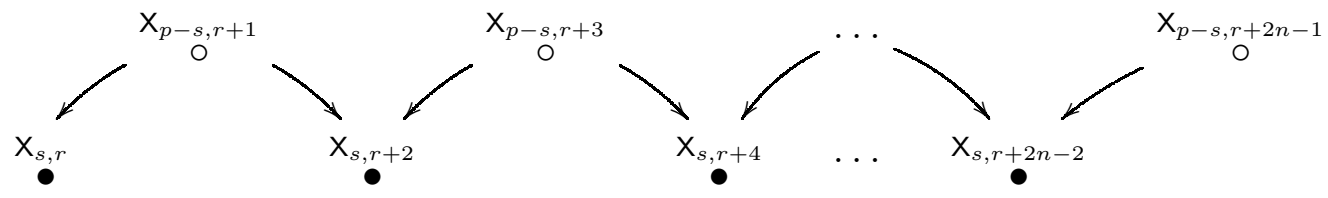

$\bigcup_{s, r}(n):$

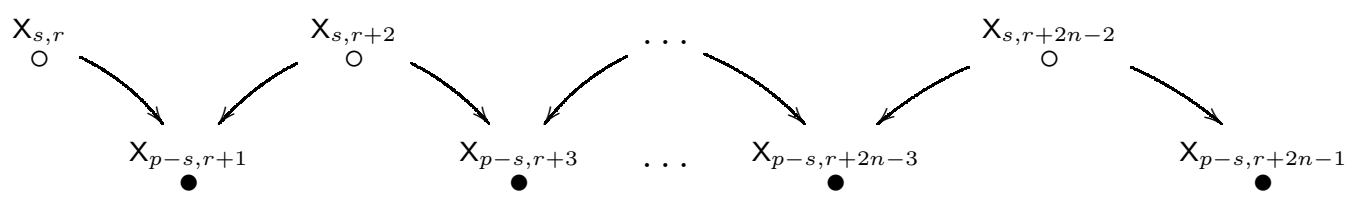

In [27], it was conjectured that the category $\mathcal{C}_{p}^{+}$is equivalent as a tensor category to the category of Virasoro algebra representations appearing in $\mathcal{L} \mathcal{M}(1, p)$. Under the equivalence, irreducible and projective modules are identified in the following way

$$
\begin{gathered}
\mathrm{X}_{p, 2 r-1} \rightarrow \mathcal{R}_{2 r-1}^{0}, \quad \mathrm{X}_{p, 2 r} \rightarrow \mathcal{R}_{2 r}^{0}, \quad \mathrm{P}_{s, 2 r-1} \rightarrow \mathcal{R}_{2 r-1}^{p-s}, \quad \mathrm{P}_{p-s, 2 r} \rightarrow \mathcal{R}_{2 r}^{s}, \\
\mathrm{X}_{s, 2 r-1} \rightarrow(2 r-1, s), \quad \mathrm{X}_{s, 2 r} \rightarrow(2 r, s), \quad 1 \leqslant s \leqslant p, \quad r \geqslant 1,
\end{gathered}
$$


where $(r, s)$ are the irreducible Virasoro modules with the highest weights

$$
\Delta_{r, s}=\left((p r-s)^{2}-(p-1)^{2}\right) / 4 p
$$

and the $\mathcal{R}_{r}^{s}$ are logarithmic Virasoro modules] (also known as staggered modules [52]), in notations of [48]. Under this identification, the fusion of the irreducible and staggered $\mathcal{V}_{p^{-}}$ modules is given by the tensor products of the corresponding $\mathcal{L} \mathcal{U}_{\mathfrak{q}} s \ell(2)$-modules.

Let $\mathcal{F}_{s, m}$, with $1 \leqslant s \leqslant p$ and $m \in \mathbb{Z}$, be the Feigin-Fuchs module [50] over $\mathcal{V}_{p}$ with the lowest conformal dimension $\Delta_{m, p-s}=\Delta_{1-m, s}$ (see precise definitions in App. A). Using the identifications for irreducible modules in (1.6), the indecomposable modules (1.2)-(1.5) over $\mathcal{L} \mathcal{U}_{\mathfrak{q}} s \ell(2)$ are then identified with quotients and submodules of the Feigin-Fuchs modules. The two families of the W-type modules from (1.2) and of the N-type from (1.4) have the correspondence

$$
\begin{array}{r}
\mathrm{W}_{s, r}(n) \rightarrow \mathcal{F}_{p-s, r} / \mathcal{F}_{p-s,-r-2 n}, \\
\mathrm{~N}_{s, r}(n) \rightarrow \mathcal{F}_{s, 1-r} / \mathcal{F}_{s, 1-r-2 n} .
\end{array}
$$

Modules from the other two families, the M-type modules from (1.3) and the $И$-type from (1.5), are contragredient to the $\mathrm{W}$ - and $\mathrm{N}$-type modules, respectively. Equivalently, they are identified with submodules in Feigin-Fuchs modules: each $\eta_{s, r}(n)$ consists of $2 n$ subquotients of $\mathcal{F}_{p-s, r}$ in Fig. 1, see App. $\mathbf{A}$, that appear from left to right; each $\mathrm{M}_{s, r}(n)$ consists of $2 n+1$ subquotients of $\mathcal{F}_{s, 1-r}$. We call these quotients and submodules of the Feigin-Fuchs modules by Kac modules.

Having the identification between subquotient structures of indecomposable qunatum-group modules and Kac modules, it is interesting to note that dimensions of subquotients over $\mathcal{L} \mathcal{U}_{\mathfrak{q}} s \ell(2)$ count conformal levels of corresponding (sub)singular vectors in the Kac modules. Recall that a singular vector satisfies the highest-weight conditions and it thus belongs to the left-most node or to any node of the type ' $\bullet$ ' in our diagrams while a subsingular vector satisfies the highest-weight conditions only in a quotient by one of the •-submodules, i.e. it belongs to a subquotient labeled by ' $O$ ' in our diagrams for modules. Then, the conformal level of a (sub)singular vector in a Kac module is given by the sum of dimensions of all irreducible quantum-group subquotients that are on the left from the subquotient of the (sub)singular vector in the corresponding diagram for $\mathcal{L} \mathcal{U}_{\mathfrak{q}} s \ell(2)$.

1.1.1. Remark. On the category $\mathrm{C}_{p}^{+}$, there is a functor ${ }^{*}$ which maps each object to its contragredient one, with all arrows reversed. In particular, it acts on indecomposable modules as $\mathrm{X}_{s, r}^{*}=\mathrm{X}_{s, r}, \mathrm{P}_{s, r}^{*}=\mathrm{P}_{s, r}, \mathrm{~N}_{s, r}(n)^{*}=\boldsymbol{U}_{s, r}(n), \boldsymbol{U}_{s, r}(n)^{*}=\mathrm{N}_{s, r}(n), \mathrm{M}_{s, r}(n)^{*}=\mathrm{W}_{s, r}(n)$ and $\mathrm{W}_{s, r}(n)^{*}=\mathrm{M}_{s, r}(n)$. In addition, the functor ${ }^{*}$ is a tensor functor

$$
(X \otimes Y)^{*}=X^{*} \otimes Y^{*} .
$$

\footnotetext{
${ }^{3}$ We note that in order to define $\mathcal{R}_{r}^{s}$, for $r>1$, as Virasoro modules it is necessary to say about the value of so-called $\beta$-invariant [52] for these modules. To determine these numbers is out of the scope of the paper.
} 
We now describe tensor product decompositions for all indecomposable modules over $\mathcal{L} \mathcal{U}_{\mathfrak{q}} s \ell(2)$. Formulas for tensor products of indecomposble modules in $\mathrm{C}_{p}^{+}$are quite cumbersome and to write them we introduce the following notation

$$
\begin{gathered}
\gamma_{1}=\left(s_{1}+s_{2}+1\right) \bmod 2, \quad \gamma_{2}=\left(s_{1}+s_{2}+p+1\right) \bmod 2, \\
\bigoplus_{r=a}^{b} f(r)=\bigoplus_{r=a}^{b}\left(1-\frac{1}{2} \delta_{r, a}-\frac{1}{2} \delta_{r, b}\right) f(r), \\
\operatorname{sg}(r)=\left\{\begin{array}{rr}
1, & r>0, \\
0, & r=0, \\
-1, & r<0 .
\end{array}\right.
\end{gathered}
$$

We do not write all possible tensor products because there are simple rules, which use commutativity and associativity of the tensor product, giving all tensor products from base ones the tensor products of simplest indecomposables, like $\mathrm{N}_{s, r}(1)$, with irreducible modules and of the simplest indecomposables with themselves. The base tensor products are collected in the following theorem.

1.2. Theorem. (1) The tensor product of two irreducible modules with $s_{1}, s_{2}=1, \ldots, p$ and $r \in \mathbb{N}$ is

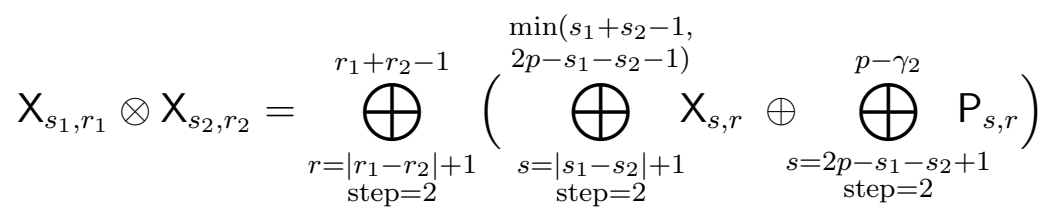

(2) The tensor product of an irreducible with a projective module with $s_{1}=1, \ldots, p$, $s_{2}=1, \ldots, p-1$ and $r \in \mathbb{N}$ is

$$
\mathrm{X}_{s_{1}, r_{1}} \otimes \mathrm{P}_{s_{2}, r_{2}}=\bigoplus_{\substack{r=\left|r_{1}-r_{2}\right|+1 \\ \text { step=2 }}}^{r_{1}+r_{2}-1}\left(\bigoplus_{\substack{s=\left|s_{1}-s_{2}\right|+1 \\ \text { step=2 }}}^{\substack{\min \left(s_{1}+s_{2}-1, 2 p-s_{1}-s_{2}-1\right)}} \bigoplus_{\substack{s=2 p-s_{1}-s_{2}+1 \\ \text { step }=2}}^{p-\gamma_{2}} \mathrm{P}_{s, r}\right) \oplus 2 \bigoplus_{\substack{r=\left|r_{1}-r_{2}\right| \\ \text { step }=2}}^{r_{1}+r_{2}} \bigoplus_{\substack{s=s_{1}+s_{2}+1 \\ \text { step }=2}}^{p-\gamma_{1}} \mathrm{P}_{s, r},
$$

where we set $\mathrm{P}_{s, 0}=0$.

(3) The tensor products of an irreducible module with simplest $\mathrm{N}$-type modules with $s_{1}, s_{2}=1, \ldots, p-1$ and $r_{1}, r_{2} \in \mathbb{N}$ are

$$
\begin{aligned}
& \mathrm{X}_{s_{1}, r_{1}} \otimes \mathrm{N}_{s_{2}, r_{2}}(1)=\bigoplus_{\substack{r=\left|r_{1}-r_{2}\right|+1 \\
\text { step }=2}}^{r_{1}+r_{2}-1} \bigoplus_{\substack{s=2 p-s_{1}-s_{2}+1 \\
\text { step }=2}}^{p-\gamma_{2}} \mathrm{P}_{s, r} \oplus \bigoplus_{\substack{r=\left|r_{1}-r_{2}-1\right|+1 \\
\text { step }=2}}^{r_{1}+r_{2}} \bigoplus_{\substack{s=p+s_{2}-s_{1}+1 \\
\text { step }=2}}^{p-\gamma_{1}} \mathrm{P}_{s, r} \oplus \\
& \oplus \bigoplus_{\substack{s=\left|s_{1}-s_{2}\right|+1 \\
\text { step }=2}}^{\substack{\min \left(s_{1}+s_{2}-1, 2 p-s_{1}-s_{2}-1\right)}} \begin{cases}\mathrm{N}_{s, r_{2}-r_{1}+1}\left(r_{1}\right), & r_{1} \leqslant r_{2} \\
\mathrm{~W}_{p-s, r_{1}-r_{2}}\left(r_{2}\right), & r_{1}>r_{2}\end{cases}
\end{aligned}
$$


(4) The tensor products of an irreducible module with simplest И-type modules with $s_{1}, s_{2}=1, \ldots, p-1$ and $r_{1}, r_{2} \in \mathbb{N}$ are

$$
\begin{aligned}
& \mathrm{X}_{s_{1}, r_{1}} \otimes \boldsymbol{U}_{s_{2}, r_{2}}(1)=\bigoplus_{\substack{r=\left|r_{1}-r_{2}\right|+1 \\
\text { step }=2}}^{r_{1}+r_{2}-1} \bigoplus_{\substack{s=2 p-s_{1}-s_{2}+1 \\
\text { step }=2}}^{p-\gamma_{2}} \mathrm{P}_{s, r} \oplus \bigoplus_{\substack{r=\left|r_{1}-r_{2}-1\right|+1 \\
\text { step }=2}}^{r_{1}+r_{2}} \bigoplus_{\substack{s=p+s_{2}-s_{1}+1 \\
\text { step }=2}}^{p-\gamma_{1}} \mathrm{P}_{s, r} \oplus \\
& \oplus \bigoplus_{\substack{s=\left|s_{1}-s_{2}\right|+1 \\
\text { step }=2}}^{\substack{\min \left(s_{1}+s_{2}-1, 2 p-s_{1}-s_{2}-1\right)}} \begin{cases}\boldsymbol{U}_{s, r_{2}-r_{1}+1}\left(r_{1}\right), & r_{1} \leqslant r_{2} \\
\mathrm{M}_{p-s, r_{1}-r_{2}}\left(r_{2}\right), & r_{1}>r_{2}\end{cases}
\end{aligned}
$$

(5) The tensor products of two simplest $\mathrm{N}$-type modules with $s_{1}, s_{2}=1, \ldots, p-1$ and $r_{1}, r_{2} \in \mathbb{N}$ are

$$
\begin{aligned}
& \mathrm{N}_{s_{1}, r_{1}}(1) \otimes \mathrm{N}_{s_{2}, r_{2}}(1)=\bigoplus_{\substack{r=\left|r_{1}-r_{2}\right|+1 \\
\text { step }=2}}^{r_{1}+r_{2}-1} \bigoplus_{\substack{s=\left|s_{1}-s_{2}\right|+1 \\
\text { step }=2}}^{p-\gamma_{2}} \mathrm{P}_{s, r} \oplus \bigoplus_{\substack{s=s_{1}+s_{2}+1 \\
\text { step }=2}}^{p-\gamma_{2}} \mathrm{P}_{s, r_{1}+r_{2}+1} \oplus \\
& \oplus \bigoplus_{\substack{r=\left|r_{1}-r_{2}+\operatorname{sg}\left(s_{2}-s_{1}\right)\right|+1 \\
\text { step=2 }}}^{r_{1}+r_{2}} \bigoplus_{\substack{s=-\left|s_{1}-s_{2}\right|+1 \\
\text { step }=2}}^{p-\gamma_{1}} \mathrm{P}_{s, r} \oplus \bigoplus_{\substack{s=\left|p-s_{1}-s_{2}\right|+1 \\
\text { step=2 }}}^{p-\left|s_{1}-s_{2}\right|-1} \mathrm{~N}_{s, r_{1}+r_{2}}(1)
\end{aligned}
$$

(6) The tensor products of two simplest И-type modules with $s_{1}, s_{2}=1, \ldots, p-1$ and $r_{1}, r_{2} \in \mathbb{N}$ are

$$
\begin{aligned}
& \eta_{s_{1}, r_{1}}(1) \otimes U_{s_{2}, r_{2}}(1)=\bigoplus_{\substack{r=\left|r_{1}-r_{2}\right|+1 \\
\text { step }=2}}^{r_{1}+r_{2}-1} \bigoplus_{\substack{s=\left|s_{1}-s_{2}\right|+1 \\
\text { step }=2}}^{p-\gamma_{2}} \mathrm{P}_{s, r} \oplus \bigoplus_{\substack{s=s_{1}+s_{2}+1 \\
\text { step }=2}}^{p-\gamma_{2}} \mathrm{P}_{s, r_{1}+r_{2}+1} \oplus \\
& \oplus \bigoplus_{\substack{r=\left|r_{1}-r_{2}+\operatorname{sg}\left(s_{2}-s_{1}\right)\right|+1 \\
\text { step }=2}}^{r_{1}+r_{2}} \bigoplus_{\substack{s=p-\left|s_{1}-s_{2}\right|+1 \\
\text { step=2 }}}^{p-\gamma_{1}} \mathrm{P}_{s, r} \oplus \bigoplus_{\substack{s=\left|p-s_{1}-s_{2}\right|+1 \\
\text { step }=2}}^{p-\left|s_{1}-s_{2}\right|-1} n_{s, r_{1}+r_{2}}(1)
\end{aligned}
$$

(7) The tensors products of simplest $\mathrm{N}$-type with simplest И-type modules with $s_{1}, s_{2}=$ $1, \ldots, p-1$ and $r_{1}, r_{2} \in \mathbb{N}$ are

$$
\begin{aligned}
& \mathrm{N}_{s_{1}, r_{1}}(1) \otimes \boldsymbol{U}_{s_{2}, r_{2}}(1)=\bigoplus_{\substack{r=\left|r_{1}-r_{2}\right|+2 \\
\text { step }=2}}^{r_{1}+r_{2}} \bigoplus_{\substack{s=\left|p-s_{1}-s_{2}\right|+1 \\
\text { step }=2}}^{p-\gamma_{1}} \mathrm{P}_{s, r} \oplus \\
& \oplus \bigoplus_{\substack{s=p-\left|s_{1}-s_{2}\right|+1 \\
\text { step }=2}}^{p-\gamma_{1}} \delta_{\mathrm{sg}\left(r_{1}-r_{2}\right), \mathrm{sg}\left(s_{1}-s_{2}\right)} \mathrm{P}_{s,\left|r_{1}-r_{2}\right|} \bigoplus_{\substack{r=\left|r_{1}-r_{2}\right|+1 \\
\text { step }=2}}^{r_{1}+r_{2}+\operatorname{sg}\left(p-s_{1}-s_{2}\right)} \bigoplus_{\substack{s=\min \left(s_{1}+s_{2}+1, 2 p-s_{1}-s_{2}+1\right) \\
\text { step }=2}}^{p-\gamma_{2}} \mathrm{P}_{s, r} \oplus
\end{aligned}
$$

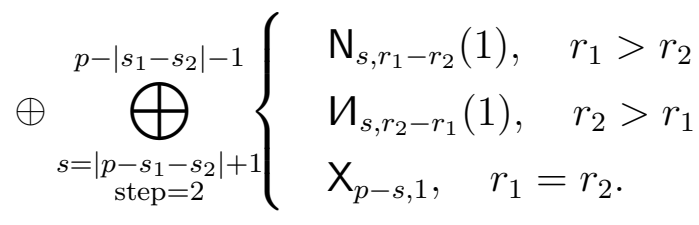


The tensor product of arbitrary two indecomposable modules can be obtained from the base tensor products given in the previous theorem and the following list of rules, see also Thm. 4.3 .

(1) The tensor product of $\mathrm{P}_{s, r}$ with an indecomposable module is isomorphic to the tensor product of $\mathrm{P}_{s, r}$ with the direct sum of all irreducible subquotients constituting the indecomposable module.

(2) An arbitrary indecomposable module of the W-, M-, N- or И-type is isomorphic to the tensor product of an irreducible module and a simplest indecomposable module:

$$
\begin{aligned}
\mathrm{N}_{s, r}(n) & =\mathrm{X}_{1, n} \otimes \mathrm{N}_{s, r+n-1}(1), \\
\boldsymbol{U}_{s, r}(n) & =\mathrm{X}_{1, n} \otimes \mathrm{U}_{s, r+n-1}(1), \\
\mathrm{W}_{s, r}(n) & =\mathrm{X}_{1, r+n} \otimes \mathrm{N}_{p-s, n}(1), \\
\mathrm{M}_{s, r}(n) & =\mathrm{X}_{1, r+n} \otimes \mathrm{U}_{p-s, n}(1),
\end{aligned}
$$

where $s=1, \ldots, p-1$ and $r, n \in \mathbb{N}$.

Thm.1.2 with these rules completes the description of the tensor structure on $\mathrm{C}_{p}^{+}$.

Following our previous result [27] about the KL equivalence established for a subcategory in $\mathrm{C}_{p}^{+}$containing all simple objects and their projective covers, we now propose the following conjecture, which was also mentioned in [27].

1.3. Conjecture. The category $\mathrm{C}_{p}^{+}$is equivalent as a tensor category to the representation category of the vertex operator algebra $\mathcal{V}_{p}$ realized in $\mathcal{L} \mathcal{M}(1, p)$.

Thus, we can conjecture fusion rules for the Kac modules over $\mathcal{V}_{p}$ using the identification in (1.8) together with Thm. 1.2 and the tensor-products rules (1) and (2) described above.

The conjecture $\mathbf{1 . 3}$ is also motivated by the fact that tensor product decompositions of the indecomposable modules in $\mathrm{C}_{p}^{+}$coincide with the fusion proposed in [49] for the Kac modules from $\mathcal{L} \mathcal{M}(1, p)$, using the identification in notations of [49]

$$
\begin{array}{r}
И_{p-s, n-r+1}(r) \rightarrow(r, s+n p), \quad \text { whenever } 2 r-1<2 n, \\
\mathrm{M}_{s, r-n}(n) \rightarrow(r, s+n p), \quad \text { whenever } 2 r-1>2 n
\end{array}
$$

and

$$
\begin{gathered}
\mathrm{N}_{p-s, n-r+1}(r) \rightarrow(r, s+n p)^{*}, \quad \text { whenever } 2 r-1<2 n \\
\mathrm{~W}_{s, r-n}(n) \rightarrow(r, s+n p)^{*}, \quad \text { whenever } 2 r-1>2 n .
\end{gathered}
$$

The paper is organised as follows. In Sec. 2, we recall a definition of the Hopf algebra $\mathcal{L} \mathcal{U}_{\mathfrak{q}} s \ell(2)$ by generators and relations and define their irreducible and projective modules. In Sec. 3, we calculate Ext ${ }^{\bullet}$ s between irreducible $\mathcal{L} \mathcal{U}_{\mathfrak{q}} s \ell(2)$-modules and obtain from this a classification theorem of all indecomposable $\mathcal{L} \mathcal{U}_{\mathfrak{q}} s \ell(2)$-modules. In Sec. 4 , we calculate 
decomposition of tensor products of all indecomposable $\mathcal{L} \mathcal{U}_{\mathfrak{q}} s \ell(2)$-modules. Sec. [5 contains our conclusions. Some technicalities and general well-known facts are arranged into six Appendices. App. A contains necessary information about Feigin-Fuchs modules over $\mathcal{V}_{p}$. App. $\mathbb{B}$ and App. $\mathbf{C}$ contain explicit description of indecomposable $\mathcal{L} \mathcal{U}_{\mathfrak{q}} s \ell(2)$-modules in terms of bases and action. App. $\mathbf{D}$ contains our result about projective resolutions for irreducible $\mathcal{L} \mathcal{U}_{\mathfrak{q}} s \ell(2)$-modules which are used in computation of Ext"'s groups. In App. $\mathbf{E}$, we give an exhaustive list of tensor products of indecomposable modules. App. $\mathbf{F}$ contains necessary information about quivers which we use to prove the classification theorem.

1.4. Notations. In the paper, $\mathbb{N}$ denotes the set of all integer $n \geqslant 1$. We also set

$$
\mathfrak{q}=e^{\frac{i \pi}{p}}
$$

for any integer $p \geqslant 2$, and use the standard notation

$$
[n]=\frac{\mathfrak{q}^{n}-\mathfrak{q}^{-n}}{\mathfrak{q}-\mathfrak{q}^{-1}}, \quad n \in \mathbb{Z}, \quad[n] !=[1][2] \ldots[n], \quad n \in \mathbb{N}, \quad[0] !=1 .
$$

For Hopf algebras, we write $\Delta, \epsilon$, and $S$ for the comultiplication, counit, and antipode respectively.

\section{CONVENTIONS AND DEFINITIONS.}

In setting the notation and recalling the basic facts about $\mathcal{L} \mathcal{U}_{\mathfrak{q}} \equiv \mathcal{L} \mathcal{U}_{\mathfrak{q}} s \ell(2)$ needed below, we largely follow [27]. We collect the definitions of different quantum groups in 2.1 and 2.2. and recall basic facts about their representation theory in 2.3 and 2.4

2.1. The restricted quantum group. The quantum group $\overline{\mathcal{U}}_{\mathfrak{q}} s \ell(2)$ is the "restricted" quantum $s \ell(2)$ with $\mathfrak{q}=e^{i \pi / p}$ and the generators $E, F$, and $K^{ \pm 1}$ satisfying the standard relations for the quantum $s \ell(2)$,

$$
K E K^{-1}=\mathfrak{q}^{2} E, \quad K F K^{-1}=\mathfrak{q}^{-2} F, \quad[E, F]=\frac{K-K^{-1}}{\mathfrak{q}-\mathfrak{q}^{-1}},
$$

with the additional relations

$$
E^{p}=F^{p}=0, \quad K^{2 p}=\mathbf{1},
$$

and the Hopf-algebra structure is given by

$$
\begin{gathered}
\Delta(E)=\mathbf{1} \otimes E+E \otimes K, \quad \Delta(F)=K^{-1} \otimes F+F \otimes \mathbf{1}, \quad \Delta(K)=K \otimes K, \\
S(E)=-E K^{-1}, \quad S(F)=-K F, \quad S(K)=K^{-1}, \\
\epsilon(E)=\epsilon(F)=0, \quad \epsilon(K)=1 .
\end{gathered}
$$

2.1.1. Central idempotents. We recall here a description of primitive central idempotents in $\overline{\mathcal{U}}_{\mathfrak{q}} s \ell(2)$ following [16]. Let $\boldsymbol{C}$ denotes the Casimir element

$$
\boldsymbol{C}=\left(\mathfrak{q}-\mathfrak{q}^{-1}\right)^{2} E F+\mathfrak{q}^{-1} K+\mathfrak{q} K^{-1}=\left(\mathfrak{q}-\mathfrak{q}^{-1}\right)^{2} F E+\mathfrak{q} K+\mathfrak{q}^{-1} K^{-1} .
$$


The $\overline{\mathcal{U}}_{\mathfrak{q}} s \ell(2)$ has $p+1$ primitive central idempotents $\boldsymbol{e}_{s}, \sum_{s=0}^{p} \boldsymbol{e}_{s}=\mathbf{1}$, which are the following polynomials in $C$ :

$$
\begin{aligned}
& \boldsymbol{e}_{s}=\frac{1}{\psi_{s}\left(\beta_{s}\right)}\left(\psi_{s}(\boldsymbol{C})-\frac{\psi_{s}^{\prime}\left(\beta_{s}\right)}{\psi_{s}\left(\beta_{s}\right)}\left(\boldsymbol{C}-\beta_{s}\right) \psi_{s}(\boldsymbol{C})\right), \quad 1 \leqslant s \leqslant p-1, \\
& \boldsymbol{e}_{0}=\frac{1}{\psi_{0}\left(\beta_{0}\right)} \psi_{0}(\boldsymbol{C}), \quad \boldsymbol{e}_{p}=\frac{1}{\psi_{p}\left(\beta_{p}\right)} \psi_{p}(\boldsymbol{C}),
\end{aligned}
$$

where $\beta_{j}=\mathfrak{q}^{j}+\mathfrak{q}^{-j}$ and

$$
\begin{aligned}
& \psi_{s}(x)=\left(x-\beta_{0}\right)\left(x-\beta_{p}\right) \prod_{j=1, j \neq s}^{p-1}\left(x-\beta_{j}\right)^{2}, \quad 1 \leqslant s \leqslant p-1, \\
& \psi_{0}(x)=\left(x-\beta_{p}\right) \prod_{j=1}^{p-1}\left(x-\beta_{j}\right)^{2}, \quad \psi_{p}(x)=\left(x-\beta_{0}\right) \prod_{j=1}^{p-1}\left(x-\beta_{j}\right)^{2} .
\end{aligned}
$$

2.2. The centralizer of $\mathcal{V}_{p}$. Here, we recall the quantum group $\mathcal{L} \mathcal{U}_{\mathfrak{q}} s \ell(2)$ (i.e. a Hopf algebra) that commutes with the Virasoro algebra $\mathcal{V}_{p}$ on the chiral space of states [27] associated with the logarithmic Virasoro models $\mathcal{L} \mathcal{M}(1, p)$.

2.2.1. Definition. The Hopf-algebra structure on $\mathcal{L} \mathcal{U}_{\mathfrak{q}} s \ell(2)$ is the following. The defining relations between the $E, F$, and $K^{ \pm 1}$ generators are the same as in $\overline{\mathcal{U}}_{\mathfrak{q}} s \ell(2)$ and given in (2.1) and (2.2), and the $e, f$, and $h$ generators have the usual $s \ell(2)$ relations

$$
[h, e]=e, \quad[h, f]=-f, \quad[e, f]=2 h,
$$

while "mixed" relations are

$$
\begin{gathered}
{[h, K]=0, \quad[E, e]=0, \quad[K, e]=0, \quad[F, f]=0, \quad[K, f]=0} \\
{[F, e]=\frac{1}{[p-1] !} K^{p} \frac{\mathfrak{q} K-\mathfrak{q}^{-1} K^{-1}}{\mathfrak{q}-\mathfrak{q}^{-1}} E^{p-1}, \quad[E, f]=\frac{(-1)^{p+1}}{[p-1] !} F^{p-1} \frac{\mathfrak{q} K-\mathfrak{q}^{-1} K^{-1}}{\mathfrak{q}-\mathfrak{q}^{-1}}} \\
{[h, E]=\frac{1}{2} E A, \quad[h, F]=-\frac{1}{2} A F}
\end{gathered}
$$

where we introduce

$$
A=\sum_{s=1}^{p-1} \frac{\left(u_{s}\left(\mathfrak{q}^{-s-1}\right)-u_{s}\left(\mathfrak{q}^{s-1}\right)\right) K+\mathfrak{q}^{s-1} u_{s}\left(\mathfrak{q}^{s-1}\right)-\mathfrak{q}^{-s-1} u_{s}\left(\mathfrak{q}^{-s-1}\right)}{\left(\mathfrak{q}^{s-1}-\mathfrak{q}^{-s-1}\right) u_{s}\left(\mathfrak{q}^{-s-1}\right) u_{s}\left(\mathfrak{q}^{s-1}\right)} u_{s}(K) \boldsymbol{e}_{s}
$$

with $u_{s}(K)=\prod_{n=1, n \neq s}^{p-1}\left(K-\mathfrak{q}^{s-1-2 n}\right)$, and $\boldsymbol{e}_{s}$ are the central primitive idempotents of $\overline{\mathcal{U}}_{\mathfrak{q}} s \ell(2)$ given in 2.1.1

The comultiplication in $\mathcal{L} \mathcal{U}_{\mathfrak{q}} s \ell(2)$ is given in (2.3) for the $E, F$, and $K$ generators and

$$
\begin{aligned}
& \Delta(e)=e \otimes 1+K^{p} \otimes e+\frac{1}{[p-1] !} \sum_{r=1}^{p-1} \frac{\mathfrak{q}^{r(p-r)}}{[r]} K^{p} E^{p-r} \otimes E^{r} K^{-r}, \\
& \Delta(f)=f \otimes 1+K^{p} \otimes f+\frac{(-1)^{p}}{[p-1] !} \sum_{s=1}^{p-1} \frac{\mathfrak{q}^{-s(p-s)}}{[s]} K^{p+s} F^{s} \otimes F^{p-s} .
\end{aligned}
$$

An explicit form of $\Delta(h)=\frac{1}{2}[\Delta(e), \Delta(f)]$ is very bulky and we do not give it here. 
The antipode $S$ and the counity $\epsilon$ are given in (2.4)-(2.5) and

$$
\begin{gathered}
S(e)=-K^{p} e, \quad S(f)=-K^{p} f, \quad S(h)=-h, \\
\epsilon(e)=\epsilon(f)=\epsilon(h)=0 .
\end{gathered}
$$

2.3. Irreducible $\mathcal{L} \mathcal{U}_{\mathfrak{q}} s \ell(2)$-modules. An irreducible $\mathcal{L} \mathcal{U}_{\mathfrak{q}} s \ell(2)$-module $\mathrm{X}_{s, r}^{ \pm}$is labeled by $( \pm, s, r)$, with $1 \leqslant s \leqslant p$ and $r \in \mathbb{N}$, and has the highest weights $\pm \mathfrak{q}^{s-1}$ and $\frac{r-1}{2}$ with respect to $K$ and $h$ generators, respectively. The $s r$-dimensional module $\mathrm{X}_{s, r}^{ \pm}$is spanned by elements $\mathrm{a}_{n, m}^{ \pm}, 0 \leqslant n \leqslant s-1,0 \leqslant m \leqslant r-1$, where $\mathrm{a}_{0,0}^{ \pm}$is the highest-weight vector and the left action of the algebra on $\mathrm{X}_{s, r}^{ \pm}$is given by

$$
\begin{array}{rlrl}
K \mathrm{a}_{n, m}^{ \pm} & = \pm \mathfrak{q}^{s-1-2 n} \mathrm{a}_{n, m}^{ \pm}, & & h \mathrm{a}_{n, m}^{ \pm}=\frac{1}{2}(r-1-2 m) \mathrm{a}_{n, m}^{ \pm}, \\
E \mathrm{a}_{n, m}^{ \pm} & = \pm[n][s-n] \mathrm{a}_{n-1, m}^{ \pm}, & e \mathrm{a}_{n, m}^{ \pm}=m(r-m) \mathrm{a}_{n, m-1}^{ \pm}, \\
F \mathrm{a}_{n, m}^{ \pm}=\mathrm{a}_{n+1, m}^{ \pm}, & & f \mathrm{a}_{n, m}^{ \pm}=\mathrm{a}_{n, m+1}^{ \pm},
\end{array}
$$

where we set $a_{-1, m}^{ \pm}=a_{n,-1}^{ \pm}=a_{s, m}^{ \pm}=a_{n, r}^{ \pm}=0$.

2.4. Projective $\mathcal{L} \mathcal{U}_{\mathfrak{q}} s \ell(2)$-modules. We now recall subquotient structure of projective covers $\mathrm{P}_{s, r}^{ \pm}$over $\mathcal{L} \mathcal{U}_{\mathfrak{q}} s \ell(2)$ introduced in [27]. The $\mathrm{P}_{s, r}^{ \pm}$module is the projective cover of $\mathrm{X}_{s, r}^{ \pm}$, for $1 \leqslant s \leqslant p-1$, and has the subquotient structure (1.1) on the left for $r=1$ and on the right for $r \geqslant 2$, where one should replace each irreducible subquotient or submodule $\mathrm{X}_{s, r+2 k-1}$ by $\mathrm{X}_{s, r+2 k-1}^{ \pm}$, and $\mathrm{X}_{s, r+2 k}$ by $\mathrm{X}_{s, r+2 k}^{ \pm}$, for any $k \geqslant 1$. The $\mathcal{L} \mathcal{U}_{\mathfrak{q}} s \ell(2)$ action is explicitly described in App. B.

A "half" of these projective modules was identified in the fusion algebra calculated in [27] with logarithmic or staggered Virasoro modules realized in $\mathcal{L} \mathcal{M}(1, p)$ models.

2.4.1. Remark. [27] We note there are no additional parameters distinguishing nonisomorphic indecomposable $\mathcal{L} \mathcal{U}_{\mathfrak{q}} s \ell(2)$-modules with the same subquotient structure as in (1.1).

2.5. Semisimple length of a module. Let $N$ be a $\mathcal{L} \mathcal{U}_{\mathfrak{q}} s \ell(2)$-module. We define a semisimple filtration of $\mathrm{N}$ as a tower of submodules

$$
\mathrm{N}=\mathrm{N}_{0} \supset \mathrm{N}_{1} \supset \ldots \supset \mathrm{N}_{l}=0
$$

such that each quotient $\mathrm{N}_{i} / \mathrm{N}_{i+1}$ is semisimple. The number $l$ is called the length of the filtration. In the set of semisimple filtrations of $\mathrm{N}$, there exists a filtration with the minimum length $\ell$. We call $\ell$ the semisimple length of $N$. The semisimple length is also known as the Loewy length and the semisimple quotients $\mathrm{N}_{i} / \mathrm{N}_{i+1}$ constitutes the so-called Loewy layers: the first Loewy layer of a module $\mathrm{N}$ is $\mathrm{N} / J(\mathrm{~N})$, where $J(\mathrm{~N})$ is the Jacobson radical of the module $\mathrm{N}$, the second Loewy layer involves taking a quotient of the radical $J(\mathrm{~N})$ by its own Jacobson radical and so on.

\footnotetext{
${ }^{4}$ A projective cover of an irreducible module is a "maximal" indecomposable module that can be mapped onto the irreducible.
} 
Evidently, an indecomposable module has the semisimple length not less than 2. Any semisimple module has the semisimple length 1.

\section{ClassificAtion OF $\mathcal{L} \mathfrak{U}_{\mathfrak{q}}$-MODUles}

To describe the category $\mathcal{C}_{p}$ of finite-dimensional $\mathcal{L} \mathcal{U}_{\mathfrak{q}} s \ell(2)$-modules, we recall first-extension groups associated with a pair of irreducible modules and give results of a computation of $n$-extensions in 3.1. This allows us to decompose the representation category $\mathcal{C}_{p}$ into full subcategories. In 3.2, we construct a family of indecomposable modules of the "FeiginFuchs" type. A classification theorem for the category $\mathcal{C}_{p}$ is presented in $\mathbf{3 . 4}$.

3.1. Extension groups. Here, we compute $n$-extensions between irreducible modules over $\mathcal{L} \mathcal{U}_{\mathfrak{q}} s \ell(2)$ using Serre-Hochschild spectral sequences associated with a filtration on $\mathcal{L} \mathcal{U}_{\mathfrak{q}} s \ell(2)$ given by the subalgebra $\overline{\mathcal{U}}_{\mathfrak{q}} s \ell(2)$ and the quotient algebra $U s \ell(2)$. We then use the extensions in order to construct four families of indecomposable modules in $\mathbf{3 . 2}$

Let $A$ and $C$ be left $\mathcal{L} \mathcal{U}_{\mathfrak{q}} s \ell(2)$-modules. We say that a short exact sequence of $\mathcal{L} \mathcal{U}_{\mathfrak{q}} s \ell(2)$ modules $0 \rightarrow A \rightarrow B \rightarrow C \rightarrow 0$ is an extension of $C$ by $A$, and we let $\operatorname{Ext}_{\mathcal{L u}_{q}}^{1}(C, A)$ denote the set of equivalence classes (see, e.g., [53]) of first extensions of $C$ by $A$. Similarly, we denote $n$-extensions by $\operatorname{Ext}_{\mathcal{L} \mathcal{U}_{q}}^{n}(C, A)$.

3.1.1. Theorem. For $1 \leqslant s \leqslant p-1$ and $\alpha= \pm$, the $n$-extension groups for $n>1$ are

$$
\operatorname{Ext}_{\mathcal{L} \mathcal{U}_{q}}^{n}\left(\mathrm{X}_{s, 1}^{\alpha}, \mathrm{X}_{s^{\prime}, r^{\prime}}^{\alpha^{\prime}}\right) \cong \begin{cases}\mathbb{C} \delta_{\alpha^{\prime}, \alpha} \delta_{s^{\prime}, s} \delta_{r^{\prime}, n+1}, & n-\text { even }, \\ \mathbb{C} \delta_{\alpha^{\prime},-\alpha} \delta_{s^{\prime}, p-s} \delta_{r^{\prime}, n+1}, & n-\text { odd }\end{cases}
$$

and for $r>1$, we have

$\operatorname{Ext}_{\mathcal{L} \mathcal{U}_{q}}^{n}\left(\mathrm{X}_{s, r}^{\alpha}, \mathrm{X}_{s^{\prime}, r^{\prime}}^{\alpha^{\prime}}\right) \cong \begin{cases}\mathbb{C} \delta_{\alpha^{\prime}, \alpha} \delta_{s^{\prime}, s}, & \text { even }<r, \text { and } r^{\prime}=r+2 k, \text { with }-\frac{n}{2} \leqslant k \leqslant \frac{n}{2}, \\ \mathbb{C} \delta_{\alpha^{\prime}, \alpha} \delta_{s^{\prime}, s}, & \text { even } n \geqslant r, \text { and } r^{\prime}=2 k, \text { with } \frac{n-r+2}{2} \leqslant k \leqslant \frac{n+r}{2}, \\ \mathbb{C} \delta_{\alpha^{\prime},-\alpha} \delta_{s^{\prime}, p-s}, & \text { odd } n<r, \text { and } r^{\prime}=r+2 k+1,-\frac{n+1}{2} \leqslant k \leqslant \frac{n-1}{2}, \\ \mathbb{C} \delta_{\alpha^{\prime},-\alpha} \delta_{s^{\prime}, p-s}, & \text { odd } n \geqslant r, \text { and } r^{\prime}=2 k+1, \frac{n-r+1}{2} \leqslant k \leqslant \frac{n+r-1}{2}, \\ 0, & \text { otherwise, }\end{cases}$

where $\delta_{a, b}$ is the Kronecker symbol and when $k$ takes half-integer values we assume it goes with the step 1.

Proof. We first recall [29] that the space $\operatorname{Ext} \frac{n}{\bar{u}_{q}}$ of $n$-extensions between irreducible modules over the subalgebra $\bar{U}_{\mathfrak{q}} s \ell(2)$ is at most $(n+1)$-dimensional and there exists a nontrivial $n$ extension only between $\mathrm{X}_{s}^{ \pm}$and $\mathrm{X}_{p-s}^{\mp}$ for odd $n$ and between $\mathrm{X}_{s}^{ \pm}$and $\mathrm{X}_{s}^{ \pm}$for even $n$, where $1 \leqslant s \leqslant p-1$ and we set $\mathrm{X}_{s}^{ \pm}=\left.\mathrm{X}_{s, 1}^{ \pm}\right|_{\bar{U}_{\mathfrak{q}} s \ell(2)}$. Moreover, there is an action of $\mathcal{L U}_{\mathfrak{q}} s \ell(2)$ on projective resolutions for simple $\overline{\mathcal{U}}_{\mathfrak{q}} s \ell(2)$-modules and this generates an action of the quotientalgebra $U s \ell(2)$ on the corresponding cochain complexes and their cohomologies. Therefore, 
for an irreducible module $X$ and an $\overline{\mathcal{U}}_{\mathfrak{q}} s \ell(2)$-module $M$, all extension groups $\operatorname{Ext}_{\bar{u}_{q}}(X, M)$ are $s \ell(2)$-modules. In particular, the space $\operatorname{Ext}_{\bar{u}_{q}}^{n}\left(\mathbf{X}_{s}^{ \pm}, \mathrm{X}_{p-s}^{\mp}\right)$ is the $(n+1)$-dimensional irreducible $s \ell(2)$-module for odd $n$.

Next, to calculate the $n$-extension groups between the simple $\mathcal{L} \mathcal{U}_{\mathfrak{q}} s \ell(2)$-modules, we use the Serre-Hochschild spectral sequence with respect to the subalgebra $\bar{U}_{\mathfrak{q}} s \ell(2)$ and the quotient algebra $U s \ell(2)$. The spectral sequence is degenerate at the second term due to the semisimplicity of the quotient algebra and we thus obtain

$$
\operatorname{Ext}_{\mathcal{L} u_{q}}^{n}\left(\mathrm{X}_{s, r}^{\alpha}, \mathrm{X}_{s^{\prime}, r^{\prime}}^{\alpha^{\prime}}\right)=H^{0}\left(U s \ell(2), \operatorname{Ext}_{\bar{u}_{q}}^{n}\left(\mathrm{X}_{s, r}^{\alpha}, \mathrm{X}_{s^{\prime}, r^{\prime}}^{\alpha^{\prime}}\right)\right),
$$

where the right-hand side is the vector space of the $s \ell(2)$-invariants in the $s \ell(2)$-module $\operatorname{Ext} \frac{n}{u_{q}}\left(\mathbf{X}_{s, r}^{\alpha}, \mathbf{X}_{s^{\prime}, r^{\prime}}^{\alpha^{\prime}}\right)$. This module is nonzero only in the cases $\alpha^{\prime}=-\alpha, s^{\prime}=p-s$ for odd $n$ and $\alpha^{\prime}=\alpha, s^{\prime}=s$ for even $n$, and isomorphic to the tensor product $\mathbf{X}_{n+1} \otimes \mathbf{X}_{r} \otimes \mathbf{X}_{r^{\prime}}$ of $s \ell(2)$ modules, where $\mathbf{X}_{r}$ is the $r$-dimensional irreducible module. Obviously, the tensor product decomposes as

$$
\mathrm{X}_{n+1} \otimes \mathrm{X}_{r} \otimes \mathrm{X}_{r^{\prime}} \cong \bigoplus_{t=\left|r-r^{\prime}\right|+1}^{r+r^{\prime}-1} \bigoplus_{k=|t-n-1|+1}^{t+n} \mathrm{X}_{k}
$$

and a simple counting of trivial $s \ell(2)$-modules in the direct sum (3.1) completes the proof.

The reader can find an alternative proof of 3.1.1 in D.2. The proof is a direct calculation involving projective resolutions. These resolutions also constitute one of our results and they are described in App. $\mathbf{D}$.

We note finally that taking $n=1$ in 3.1.1 gives an immediate consequence obtained in [27].

3.1.2. Corollary. [27] For $1 \leqslant s \leqslant p-1, r \in \mathbb{N}$ and $\alpha, \alpha^{\prime} \in\{+,-\}$, there are vector-space isomorphisms

$$
\operatorname{Ext}_{\mathcal{L}{U_{q}}_{q}}^{1}\left(\mathrm{X}_{s, r}^{\alpha}, \mathrm{X}_{s^{\prime}, r^{\prime}}^{\alpha^{\prime}}\right) \cong \begin{cases}\mathbb{C}, & \alpha^{\prime}=-\alpha, s^{\prime}=p-s, r^{\prime}=r \pm 1, \\ 0, & \text { otherwise. }\end{cases}
$$

There are no nontrivial extensions between $\mathrm{X}_{p, r}^{ \pm}$and any irreducible module.

3.2. Indecomposable modules. We now construct four infinite families of indecomposable modules over $\mathcal{L} \mathcal{U}_{\mathfrak{q}} s \ell(2)$.

Using 3.1.2 we can "glue" two irreducible modules into an indecomposable module only in the case if the irreducibles have opposite signs, different evenness in the $r$-index and the sum of the two $s$-indexes is equal to $p$. Thereby, for $1 \leqslant s \leqslant p-1$ and integers $r, n \geqslant 1$, we can introduce four types of indecomposable modules of the semisimple length 2 classified by their "shapes": W-, M-, N-, and reversed-N modules denoted by the symbol $И$. The modules are described in (1.2)-(1.5) by their subquotient structure, where $X_{1} \longrightarrow X_{2}$ denotes an extension 
by an element from the space $\operatorname{Ext}_{\mathcal{L} \mathcal{U}_{q}}^{1}\left(\mathrm{X}_{1}, \mathrm{X}_{2}\right)$, with $\mathrm{X}_{1}$ being an irreducible subquotient and $\mathrm{X}_{2}$ an irreducible submodule. The subquotient structure uniquely defines these modules, up to an isomorphism, due to the one-dimensionality of the first-extension groups (3.2). We now turn to an explicit description of these modules in terms of bases and action.

$\mathbf{W}_{s, r}^{ \pm}(\boldsymbol{n})$ : The module $\mathrm{W}_{s, r}^{ \pm}(n)$ has the subquotient structure (1.2) where each irreducible subquotient $\mathrm{X}_{s, r+2 k}$ should be replaced by $\mathrm{X}_{s, r+2 k}^{ \pm}$, and $\mathrm{X}_{s, r+2 k-1}$ by $\mathrm{X}_{s, r+2 k-1}^{\mp}$, and $n$ is the number of the bottom modules (filled dots $\bullet$ ). We first describe the $\mathcal{L} \mathcal{U}_{\mathfrak{q}} s \ell(2)$-action on a

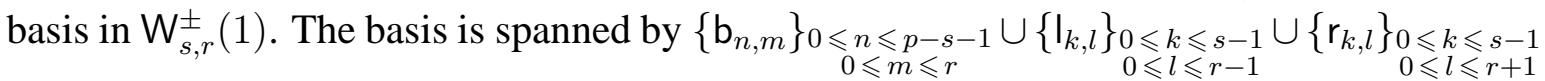
and identified with the corresponding submodule in $\mathrm{P}_{p-s, r+1}^{\mp}$ explicitly described in App.C.1. The modules $\mathrm{W}_{s, r}^{ \pm}(n)$, with $n>1$, are defined then by taking appropriate submodules in the direct sum of $n$ modules $\mathrm{W}_{s, r+2 k}^{ \pm}(1)$ :

$$
\mathrm{W}_{s, r}^{ \pm}(n) \subset \mathbf{W}_{s, r}^{ \pm}(1) \oplus \mathbf{W}_{s, r+2}^{ \pm}(1) \oplus \cdots \oplus \mathbf{W}_{s, r+2 n-2}^{ \pm}(1)
$$

where we take a basis for the subquotient $\mathrm{X}_{s, r+2 k}^{ \pm}$in $\mathrm{W}_{s, r}^{ \pm}(n)$ as the sum of the bases in the two subquotients $X_{s, r+2 k}^{ \pm}$and $X_{s, r+2 k}^{ \pm}$in the direct sum $\mathbf{W}_{s, r+2 k-2}^{ \pm}(1) \oplus \mathbf{W}_{s, r+2 k}^{ \pm}(1)$. We give an explicit action in App.C.2 in the example of the module $\mathrm{W}_{s, r}^{ \pm}(2)$.

$\mathbf{M}_{s, r}^{ \pm}(\boldsymbol{n})$ : The module $\mathrm{M}_{s, r}^{ \pm}(n)$ is defined as the contragredient module to the $\mathrm{W}_{s, r}^{ \pm}(n)$ module, which means that all the arrows in the diagram for $\mathrm{W}_{s, r}^{ \pm}(n)$ should be reversed in order to get a diagram for $\mathrm{M}_{s, r}^{ \pm}(n)$. This module has the subquotient structure (1.3) where each irreducible submodule $\mathrm{X}_{s, r+2 k}$ should be replaced by $\mathrm{X}_{s, r+2 k}^{ \pm}$, and $\mathrm{X}_{s, r+2 k-1}$ by $\mathrm{X}_{s, r+2 k-1}^{\mp}$. For later convenience, we give here its subquotient structure

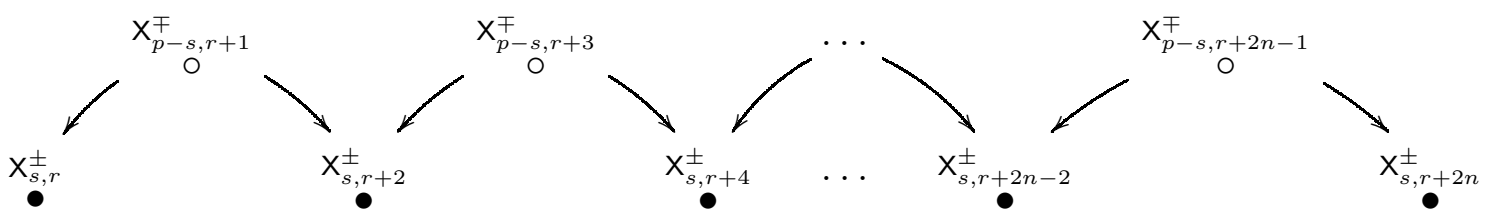

where $n$ is the number of the top modules (open dots $\circ$ ). The $\mathcal{L} \mathcal{U}_{\mathfrak{q}} s \ell(2)$-action on a basis is explicitly described in App.C.1 in the example of $\mathrm{M}_{s, r}^{ \pm}(1)$. The modules $\mathrm{M}_{s, r}^{ \pm}(n)$, with $n>1$, are defined then by taking appropriate quotients of the direct sum of $n$ modules $\mathrm{M}_{s, r+2 k}^{ \pm}(1)$ in accordance with the exact sequence

$$
0 \longrightarrow \bigoplus_{k=1}^{n-1} \mathrm{X}_{s, r+2 k}^{ \pm} \longrightarrow \bigoplus_{k=0}^{n-1} \mathrm{M}_{s, r+2 k}^{ \pm}(1) \longrightarrow \mathrm{M}_{s, r}^{ \pm}(n) \longrightarrow 0
$$

where the image of each $\mathrm{X}_{s, r+2 k}^{ \pm}$under the embedding has a basis which is the sum of the bases in the two submodules $\mathrm{X}_{s, r+2 k}^{ \pm}$and $\mathrm{X}_{s, r+2 k}^{ \pm}$in the direct sum $\mathrm{M}_{s, r+2 k-2}^{ \pm}(1) \oplus \mathrm{M}_{s, r+2 k}^{ \pm}(1)$.

$\mathbf{N}_{s, r}^{ \pm}(\boldsymbol{n})$ : The module $\mathbf{N}_{s, r}^{ \pm}(n)$ is defined as the quotient of $\mathrm{M}_{s, r}^{ \pm}(n)$ by its submodule $\mathrm{X}_{s, r+2 n}^{ \pm}$. The $\mathrm{N}_{s, r}^{ \pm}(n)$ has the subquotient structure (1.4) where each irreducible submodule $\mathrm{X}_{s, r+2 k}$ is replaced by $\mathrm{X}_{s, r+2 k}^{ \pm}$, and $\mathrm{X}_{s, r+2 k-1}$ by $\mathrm{X}_{s, r+2 k-1}^{\mp}$ and $n$ is the number of the top modules (open dots $\circ$ ) and at the same time the number of the bottom modules (filled dots $\bullet$ ). 
The $\mathcal{L} \mathcal{U}_{\mathfrak{q}} s \ell(2)$-action on a basis in $\mathrm{N}_{s, r}^{ \pm}(n)$ is explicitly described in C.1 in the example of the Weyl module $\mathrm{N}_{s, r}^{ \pm}(1)$.

$И_{s, r}^{ \pm}(\boldsymbol{n})$ : The module $И_{s, r}^{ \pm}(n)$ is defined as the contragredient module to the $\mathrm{N}_{s, r}^{ \pm}(n)$ module defined just above, i.e. one should reverse all the arrows. It has the subquotient structure (1.5) where one should replace each irreducible subquotient $\mathrm{X}_{s, r+2 k}$ by $\mathrm{X}_{s, r+2 k}^{ \pm}$, and $\mathrm{X}_{s, r+2 k-1}$ by $\mathrm{X}_{s, r+2 k-1}^{\mp}$, and $n$ is the number of the bottom modules (filled dots $\bullet$ ) and at the same time the number of the top modules (open dots $\circ$ ). The $\mathcal{L} \mathcal{U}_{\mathfrak{q}} s \ell(2)$-action on a basis in $И_{s, r}^{ \pm}(n)$ is explicitly described in $\mathbf{C . 1}$ in the example of $И_{s, r}^{ \pm}(1)$.

The introduced four infinite series of indecomposable modules $\mathrm{W}_{s, r}^{ \pm}(n), \mathrm{M}_{s, r}^{ \pm}(n), \mathrm{N}_{s, r}^{ \pm}(n)$, and $\boldsymbol{U}_{s, r}^{ \pm}(n)$ are then used in construction of the projective resolutions and involved in a oneto-one correspondence with the Kac modules over Virasoro.

3.3. Classification theorem for the category $\mathcal{C}_{p}$. Here, we describe the category $\mathcal{C}_{p}$ of finite-dimensional modules over $\mathcal{L} \mathcal{U}_{\mathfrak{q}} s \ell(2)$. We use results about possible extensions between irreducible modules, Thm. 3.1.1 and the list of indecomposable modules proposed above to state the following classification theorem.

3.4. Theorem. Let $\mathcal{C}_{p}$ denotes the category of finite-dimensional $\mathcal{L} \mathcal{U}_{\mathfrak{q}} s \ell(2)$-modules. Then, we have the following:

(1) The category $\mathrm{C}_{p}$ has the decomposition

$$
\mathrm{C}_{p}=\bigoplus_{s=1}^{p-1}\left(\mathrm{C}^{+}(s) \oplus \mathrm{C}^{-}(s)\right) \oplus \bigoplus_{r \geqslant 1}\left(\mathcal{S}^{+}(r) \oplus \mathcal{S}^{-}(r)\right),
$$

where each direct summand is a full indecomposable subcategory.

(2) Each of the full subcategories $\mathcal{S}^{+}(r)$ and $\mathcal{S}^{-}(r)$ is semisimple and contains precisely one irreducible module, $\mathrm{X}_{p, r}^{+}$and $\mathrm{X}_{p, r}^{-}$respectively.

(3) Each full subcategory $\mathrm{C}^{+}(s)$ contains the infinite family of irreducible modules $\mathrm{X}_{s, 2 r-1}^{+}$ and $\mathrm{X}_{p-s, 2 r}^{-}$, with $r \in \mathbb{N}$, and the following set of indecomposable modules:

- the projective modules $\mathrm{P}_{s, 2 r-1}^{+}$and $\mathrm{P}_{p-s, 2 r}^{-}$, where $r \in \mathbb{N}$;

- four series of indecomposable modules, for all integer $n \geqslant 1$ and $r \in \mathbb{N}$, given by

- the $\mathrm{W}_{s, 2 r-1}^{+}(n)$ and $\mathrm{W}_{p-s, 2 r}^{-}(n)$ modules and the contragredient to them $\mathrm{M}_{s, 2 r-1}^{+}(n)$ and $\mathrm{M}_{p-s, 2 r}^{-}(n)$ modules;

- the $\mathrm{N}_{s, 2 r-1}^{+}(n)$ and $\mathrm{N}_{p-s, 2 r}^{-}(n)$ modules and the contragredient to them $\eta_{s, 2 r-1}^{+}(n)$ and $И_{p-s, 2 r}^{-}(n)$ modules.

(4) Each full subcategory $\mathrm{C}^{-}(s)$ contains the infinite family of irreducible modules $\mathrm{X}_{s, 2 r}^{+}$ and $\mathrm{X}_{p-s, 2 r-1}^{-}$, with $r \in \mathbb{N}$, and the following set of indecomposable modules:

- the projective modules $\mathrm{P}_{s, 2 r}^{+}$and $\mathrm{P}_{p-s, 2 r-1}^{-}$, where $r \in \mathbb{N}$;

- four series of indecomposable modules, for all integer $n \geqslant 1$ and $r \in \mathbb{N}$, given by

- the $\mathrm{W}_{s, 2 r}^{+}(n)$ and $\mathrm{W}_{p-s, 2 r-1}^{-}(n)$ modules and the contragredient to them $\mathrm{M}_{s, 2 r}^{+}(n)$ and $\mathrm{M}_{p-s, 2 r-1}^{-}(n)$ modules; 
- the $\mathrm{N}_{s, 2 r}^{+}(n)$ and $\mathrm{N}_{p-s, 2 r-1}^{-}(n)$ modules and the contragredient to them $\eta_{s, 2 r}^{+}(n)$ and $\boldsymbol{И}_{p-s, 2 r-1}^{-}(n)$ modules.

This exhausts the list of indecomposable modules in $\mathrm{C}^{+}(s)$ and $\mathrm{C}^{-}(s)$.

The strategy of the proof is as follows. We first note that all projective modules in $\mathcal{C}_{p}$ are injective modules. This information suffices to ensure that indecomposable modules with semisimple (Loewy) length 3 are projective modules and that there are no modules with semisimple length 4 or more. Therefore, to complete the proof of 3.4, it remains to classify indecomposable modules with semisimple length 2 . We do this in $\mathbf{3 . 5}$ using a correspondence between modules with semisimple length 2 and indecomposable representations of the quivers $A_{N}$, for appropriate $N$.

We now turn to a proof of Thm. 3.4. We remind first the following fact easily established using the identity $\operatorname{Ext}_{\mathcal{L} \mathcal{U}_{q}}^{n}(\mathrm{P}, \mathrm{M})=0$ for a projective module $\mathrm{P}$ and any module $\mathrm{M}$.

\subsubsection{Proposition. [27]}

(1) Every indecomposable $\mathcal{L} \mathcal{U}_{\mathfrak{q}} s \ell(2)$-module with the semisimple length 3 is isomorphic to $\mathrm{P}_{s, r}^{ \pm}$, for some $s \in\{1,2, \ldots, p-1\}$ and some finite $r \in \mathbb{N}$.

(2) There are no indecomposable modules with the semisimple length greater than 3.

3.5. Modules with semisimple length 2. To complete the proof of the parts (3) and (4) in 3.4, it remains to classify finite-dimensional modules with the semisimple length $\ell=2$. We restrict our classification to the subcategory $\mathcal{C}_{p}^{+}$because the full category $\mathcal{C}_{p}$ is decomposed as $\mathcal{C}_{p}^{+} \oplus \mathcal{C}_{p}^{-}$where the two summands are equivalent as abelian categories. Indeed, the functor $\nu: \mathcal{C}_{p}^{+} \rightarrow \mathcal{C}_{p}^{-}$mentioned in the introduction section and defined by $\mathrm{X}_{s, 2 r-1}^{+} \mapsto \mathrm{X}_{s, 2 r-1}^{-}$, and $\mathrm{X}_{s, 2 r}^{-} \mapsto \mathrm{X}_{s, 2 r}^{+}$, and similarly for all indecomposable objects, gives an equivalence between the abelian categories $\mathrm{C}_{p}^{+}$and $\mathrm{C}_{p}^{-}$.

3.5.1. The category $\mathrm{C}^{(2)}(s)$. For $1 \leqslant s \leqslant p-1$, let $\mathrm{C}^{(2)}(s)$ be the full subcategory of $\mathrm{C}^{+}(s)$ consisting of $\mathcal{L} \mathcal{U}_{\mathfrak{q}} s \ell(2)$-modules with semisimple length $\ell \leqslant 2$. The set of simple objects in $\mathrm{C}^{(2)}(s)$ consist of the infinite family of irreducible modules $\mathrm{X}_{s, 2 r-1}^{+}$and $\mathrm{X}_{p-s, 2 r}^{-}$, with $r \geqslant 1$. Obviously, any module in $\mathfrak{C}^{(2)}(s)$ can be obtained either by

- the extension of a finite direct sum of semisimple modules $n_{r} \mathrm{X}_{s, 2 r-1}^{+}$, with $r \geqslant 1$ and multiplicities $n_{r} \geqslant 0$, by a direct sum of $m_{r^{\prime}} \mathbf{X}_{p-s, 2 r^{\prime}}^{-}$, with $r^{\prime} \geqslant 1$ and multiplicities $m_{r^{\prime}} \geqslant 0$, via a direct sum of $x^{+} \in \operatorname{Ext}_{\mathcal{L} u_{q}}^{1}\left(n_{r} \mathbf{X}_{s, 2 r-1}^{+}, m_{r^{\prime}} \mathbf{X}_{p-s, 2 r^{\prime}}^{-}\right)$

or by

- the extension of a finite direct sum of semisimple modules $m_{r^{\prime}} \mathbf{X}_{p-s, 2 r^{\prime}}^{-}$by a direct sum of modules $n_{r} \mathbf{X}_{s, 2 r-1}^{+}$via a direct sum of $x^{-} \in \operatorname{Ext}_{\mathcal{L} \mathcal{U}_{q}}^{1}\left(m_{r^{\prime}} \mathbf{X}_{p-s, 2 r^{\prime}}^{-}, n_{r} \mathbf{X}_{s, 2 r-1}^{+}\right)$. 
For any finite set of multiplicities $\left\{m_{r}, n_{r} ; r \geqslant 1, m_{r}, n_{r} \geqslant 0\right\}$, we choose an extension $X^{+} \in \operatorname{Ext}_{\mathcal{L u}_{q}}^{1}\left(\bigoplus_{r \geqslant 1} n_{r} \mathrm{X}_{s, 2 r-1}^{+}, \bigoplus_{r \geqslant 1} m_{r} \mathrm{X}_{p-s, 2 r}^{-}\right)$. Let $\operatorname{Ex}_{X^{+}}\left(n_{r}, m_{r}\right) \in \mathrm{Ob}\left(\mathcal{C}^{(2)}(s)\right)$ denotes the module defined by the extension $X^{+}$,

$$
\mathrm{Ex}_{X^{+}}\left(n_{r}, m_{r}\right): \quad \bigoplus_{r \geqslant 1} n_{r} \mathrm{x}_{s, 2 r-1}^{+} \stackrel{X^{+}}{\longrightarrow} \bigoplus_{r \geqslant 1} m_{r} \mathrm{X}_{p-s, 2 r}^{-}
$$

where we denote the dependence on the set of the multiplicties $\left\{n_{r}, m_{r}\right\}$ in the round brackets, and omit braces and range for the $r$-index for brevity. We define the modules $\operatorname{Ex}_{X^{-}}\left(m_{r}, n_{r}\right)$ similarly taking $X^{-} \in \operatorname{Ext}_{\mathcal{L} \mathcal{U}_{q}}^{1}\left(\bigoplus_{r \geqslant 1} m_{r} \mathrm{X}_{p-s, 2 r}^{-}, \bigoplus_{r \geqslant 1} n_{r} \mathrm{X}_{s, 2 r-1}^{+}\right)$. The modules $\mathrm{W}_{s, 2 r^{\prime}-1}^{+}(n)$ and $\boldsymbol{U}_{s, 2 r^{\prime}-1}^{+}(n)$ introduced in $\mathbf{3 . 2}$ are particular cases of the $\operatorname{Ex}_{X^{+}}\left(n_{r}, m_{r}\right)$ modules: the $И_{s, 2 r^{\prime}-1}^{+}(n)$ corresponds to the multiplicities $n_{r}=m_{r}=1$ in (3.3) for $r^{\prime} \leqslant r \leqslant r^{\prime}+n-1$ and they are zero otherwise.

Using 3.1.2, we note that a module $\operatorname{Ex}_{X^{ \pm}}\left(n_{r}, m_{r^{\prime}}\right)$ is a direct sum of two or more indecomposables if there exist two (or more) subsets, each indexed by $\left(r, r^{\prime}\right)$, in the set of non-zero values of the multiplicities $n_{r}, m_{r^{\prime}}$ such that they are separated in the $r$ - or $r^{\prime}$-index by the value 2 or more. In order to calssify all indecomposable modules, we will thus restrict to the following choice of the multiplicities $n_{r}, m_{r^{\prime}}$ : they are non-zero for all numbers in regions $1 \leqslant k \leqslant r \leqslant k^{\prime}$ and $2 \leqslant l \leqslant r^{\prime} \leqslant l^{\prime}$, where $l=k \pm 1$ and $l^{\prime}=k^{\prime} \pm 1$, and the multiplicities are zero otherwise.

We define next full subcategories $\mathrm{C}_{n}^{(2),+}(s)$ and $\mathrm{C}_{n}^{(2),-}(s)$, for $n \geqslant 1$, generating a filtration of the category $\mathcal{C}^{(2)}(s)$ as follows. Isomorphism classes of simple objects from $\mathcal{C}_{n}^{(2),+}(s)$ consist of the set $\left\{\mathrm{X}_{s, 2 r-1}^{+}, \mathrm{X}_{p-s, 2 r}^{-} ; 1 \leqslant r \leqslant n\right\}$. Any object of $\mathcal{C}_{n}^{(2),+}(s)$ is either a semisimple module or a module $\mathrm{N}$ such that $\mathrm{N} / \mathrm{N}_{1}=\bigoplus_{r=1}^{n} n_{r} \mathrm{X}_{s, 2 r-1}^{+}$for appropriate $n_{r} \geqslant 0$, where $\mathrm{N}_{1}$ is the maximal semisimple submodule (the socle); in other words, an object of $\mathcal{C}_{n}^{(2),+}(s)$ is a module $\mathrm{Ex}_{X^{+}}\left(n_{r}, m_{r}\right)$ introduced in (3.3) with $n_{r}=m_{r}=0$ for $r>n$. Objects of $\mathrm{C}_{n}^{(2),-}(s)$ are defined similarly with $\mathrm{N} / \mathrm{N}_{1}=\bigoplus_{r=1}^{n} m_{r} \mathrm{X}_{p-s, 2 r}^{-}$, with some $m_{r} \geqslant 0$. We note that we have the filtration by full abelian subcategories

$$
\mathrm{C}_{1}^{(2), \pm}(s) \subset \mathcal{C}_{2}^{(2), \pm}(s) \subset \mathcal{C}_{3}^{(2), \pm}(s) \subset \cdots \subset \mathfrak{C}^{(2)}(s),
$$

with $\mathrm{Ob}\left(\mathrm{C}^{(2)}(s)\right)=\cup_{n \geqslant 1} \mathrm{Ob}\left(\mathrm{C}_{n}^{(2), \pm}(s)\right)$.

We now reduce the classification of modules with semisimple length 2 in each $\mathrm{C}_{n}^{(2), \pm}(s)$ to the classification of indecomposable representations of the $A_{2 n}$-type quivers $\mathbf{A}_{2 n}$. The reader is referred to [54, 55] and Appendix $\mathbf{F}$ for the necessary facts about quivers.

3.5.2. Lemma. Each of the abelian categories $\mathrm{C}_{n}^{(2),+}(s)$ and $\mathrm{C}_{n}^{(2),-}(s)$ is equivalent to the category $\operatorname{Rep}\left(\mathbf{A}_{2 n}\right)$ of representations of the $A_{2 n}$-type quiver $\mathbf{A}_{2 n}$.

Proof. The lemma is based on an observation that morphisms $\varepsilon_{r}$, with $1 \leqslant r \leqslant 2 n-1$, together with objects in

$$
\mathrm{X}_{s, 1}^{+} \stackrel{\varepsilon_{1}}{\longrightarrow} \mathrm{M}_{s, 1}^{+}(1) \stackrel{\varepsilon_{2}}{\longleftarrow} \mathrm{X}_{s, 3}^{+} \stackrel{\varepsilon_{3}}{\longrightarrow} \mathrm{M}_{s, 3}^{+}(1) \stackrel{\varepsilon_{4}}{\longleftarrow} \ldots \stackrel{\varepsilon_{2 n-4}}{\longleftarrow} \mathrm{X}_{s, 2 n-3}^{+} \stackrel{\varepsilon_{2 n-3}}{\longrightarrow}
$$




$$
\stackrel{\varepsilon_{2 n-3}}{\longrightarrow} \mathrm{M}_{s, 2 n-3}^{+}(1) \stackrel{\varepsilon_{2 n-2}}{\longleftarrow} \mathrm{X}_{s, 2 n-1}^{+} \stackrel{\varepsilon_{2 n-1}}{\longrightarrow} \mathrm{N}_{s, 2 n-1}^{+}(1)
$$

make up a quiver $\mathbf{A}_{2 n}$ in the category $\mathrm{C}_{n}^{(2),-}(s)$; a similar collection of objects and morphisms (one should replace each $\mathrm{X}_{s, 2 r-1}^{+}$by $\mathrm{X}_{p-s, 2 r}^{-}, \mathrm{M}_{s, 2 r-1}^{+}(1)$ by $\mathrm{M}_{p-s, 2 r}^{-}(1)$, and $\mathrm{N}_{s, 2 n-1}^{+}(1)$ by $\boldsymbol{U}_{s, 1}^{+}(1)$ and take all morphisms between these objects) make up a quiver $\mathbf{A}_{2 n}$ in the category $\mathcal{C}_{n}^{(2),+}(s)$. We take then the functors of Hom to each of the two categories, $\mathcal{C}_{n}^{(2), \pm}(s)$ and $\operatorname{Rep}\left(\mathbf{A}_{2 n}\right)$, to establish an equivalence. The equivalence, e.g., between the categories $\mathfrak{C}_{n}^{(2),-}(s)$ and $\operatorname{Rep}\left(\mathbf{A}_{2 n}\right)$ is given by the functor $\mathcal{F}$ that acts on objects as

$$
\mathcal{F}\left(\operatorname{Ex}_{X^{-}}\left(m_{r}, n_{r}\right)\right)=\left(\left(V_{1}, V_{2}, \ldots, V_{2 n}\right), f_{j, j \pm 1}\right)
$$

where

$$
\begin{array}{ll}
V_{2 r-1}=\operatorname{Hom}_{\mathcal{L} \mathcal{U}_{q}}\left(\mathrm{X}_{s, 2 r-1}^{+}, \operatorname{Ex}_{X^{-}}\left(m_{r}, n_{r}\right)\right)=\mathbb{C}^{n_{r}}, & 1 \leqslant r \leqslant n, \\
V_{2 r}=\operatorname{Hom}_{\mathcal{L} \mathcal{U}_{q}}\left(\mathrm{M}_{s, 2 r-1}^{+}(1), \operatorname{Ex}_{X^{-}}\left(m_{r}, n_{r}\right)\right)=\mathbb{C}^{m_{r}}, & 1 \leqslant r \leqslant n-1, \\
V_{2 n}=\operatorname{Hom}_{\mathcal{L} \mathcal{U}_{q}}\left(\mathrm{~N}_{s, 2 n-1}^{+}(1), \operatorname{Ex}_{X^{-}}\left(m_{r}, n_{r}\right)\right)=\mathbb{C}^{m_{n}} &
\end{array}
$$

and, for linearly independent homomorphisms $\varepsilon_{2 r-1}, \varepsilon_{2 r} \in \operatorname{Hom}_{\mathcal{L u}_{q}}\left(\mathrm{X}_{s, 2 r \pm 1}^{+}, \mathrm{M}_{s, 2 r-1}^{+}(1)\right)=\mathbb{C}$, the linear maps $f_{2 r, 2 r \pm 1} \in \operatorname{Hom}_{\mathbb{C}}\left(V_{2 r}, V_{2 r \pm 1}\right)$ are defined as

$$
f_{2 r, 2 r-1}(\varphi)=\varphi \circ \varepsilon_{2 r-1}, \quad f_{2 r, 2 r+1}(\varphi)=\varphi \circ \varepsilon_{2 r},
$$

for each $\varphi \in V_{2 r}$, and with the natural action on morphisms.

The existence of a functor $\mathcal{G}$ such that both $\mathcal{G F}$ and $\mathcal{F G}$ are the identity functors is evident from the definitions of the categories $\mathcal{C}_{n}^{(2),-}(s)$ and $\operatorname{Rep}\left(\mathbf{A}_{2 n}\right)$.

Propositions 3.5.2 and F.2.1 immediately imply the desired classification of finite-dimensional $\mathcal{L} \mathcal{U}_{\mathfrak{q}} s \ell(2)$-modules with semisimple length $\ell=2$, thus completing the proof of Thm. 3.4.

We now turn to the most important part of the paper which presents tensor product decompositions of all indecomposable modules over $\mathcal{L} \mathcal{U}_{\mathfrak{q}} s \ell(2)$.

\section{TENSOR PRODUCT DECOMPOSITIONS}

To formulate the main result of the paper, we remind [27] that the tensor products between irreducible $\mathcal{L} \mathcal{U}_{\mathfrak{q}} s \ell(2)$-modules are

$$
\mathrm{X}_{s_{1}, r_{1}}^{\alpha} \otimes \mathrm{X}_{s_{2}, r_{2}}^{\beta}=\bigoplus_{\substack{r=\left|r_{1}-r_{2}\right|+1 \\ \text { step }=2}}^{r_{1}+r_{2}-1}\left(\bigoplus_{\substack{s=\left|s_{1}-s_{2}\right|+1 \\ \text { step=2 }}}^{\substack{\min \left(s_{1}+s_{2}-1, 2 p-s_{1}-s_{2}-1\right)}} \bigoplus_{\substack{s, r \\ s=2 p-s_{1}-s_{2}+1 \\ \text { step }=2}}^{p-\gamma_{2}} \mathrm{P}_{s, r}^{\alpha \beta}\right),
$$

with $1 \leqslant s_{1}, s_{2} \leqslant p-1$, and $r_{1}, r_{2} \geqslant 1$, and $\alpha, \beta= \pm$.

We also refer the reader to (1.10) which collect some notations we use here intensively. 
The following theorem states decompositions of tensor products of irreducible modules $\mathrm{X}_{s_{1}, r_{1}}^{\alpha}$ with the (contragredient) Weyl modules $\mathrm{N}_{s_{2}, r_{2}}^{\beta}(1)$ and $\boldsymbol{U}_{s_{2}, r_{2}}^{\beta}(1)$.

4.1. Theorem. For $1 \leqslant s_{1}, s_{2} \leqslant p-1, r_{1}, r_{2} \geqslant 1$, and $\alpha, \beta= \pm$, we have isomorphisms of $\mathcal{L} \mathcal{U}_{\mathfrak{q}} s \ell(2)$-modules

$$
\begin{aligned}
& \mathrm{X}_{s_{1}, r_{1}}^{\alpha} \otimes \mathrm{N}_{s_{2}, r_{2}}^{\beta}(1)=\bigoplus_{\substack{r=\left|r_{1}-r_{2}\right|+1 \\
\text { step }=2}}^{r_{1}+r_{2}-1} \bigoplus_{\substack{s=2 p-s_{1}-s_{2}+1 \\
\text { step }=2}}^{p-\gamma_{2}} \mathrm{P}_{s, r}^{\alpha \beta} \oplus \bigoplus_{\substack{r=\left|r_{1}-r_{2}-1\right|+1 \\
\text { step }=2}}^{r_{1}+r_{2}} \bigoplus_{\substack{s=p+s_{2}-s_{1}+1 \\
\text { step }=2}}^{p-\gamma_{1}} \mathrm{P}_{s, r}^{-\alpha \beta} \\
& \bigoplus \bigoplus_{\substack{s=\left|s_{1}-s_{2}\right|+1 \\
\text { step }=2}}^{\substack{\min \left(s_{1}+s_{2}-1, 2 p-s_{1}-s_{2}-1\right)}} \begin{cases}\mathrm{N}_{s, r_{2}-r_{1}+1}^{\alpha \beta}\left(r_{1}\right), & r_{1} \leqslant r_{2} \\
\mathrm{~W}_{p-s, r_{1}-r_{2}}^{-\alpha \beta}\left(r_{2}\right), & r_{1}>r_{2}\end{cases}
\end{aligned}
$$

and the tensor product with the module contragredient to $\mathrm{N}_{s_{2}, r_{2}}^{\beta}(1)$ is

$$
\mathbf{X}_{s_{1}, r_{1}}^{\alpha} \otimes \boldsymbol{U}_{s_{2}, r_{2}}^{\beta}(1)=\left(\mathbf{X}_{s_{1}, r_{1}}^{\alpha} \otimes \mathbf{N}_{s_{2}, r_{2}}^{\beta}(1)\right)^{*},
$$

where we set $\mathrm{P}^{*} \equiv \mathrm{P}, \mathrm{N}^{*} \equiv И$, and $\mathrm{W}^{*} \equiv \mathrm{M}$.

Proof. We consider first the tensor product in (4.2). Let the set $\left\{a_{n^{\prime}, m^{\prime}}\right\}$ denotes the basis in the first tensorand with the action described in 2.3. The second tensorand has the basis $\left\{\mathrm{b}_{n, m}\right\} \cup\left\{\mathrm{r}_{k, l}\right\}$, see (C.3), with the $\mathcal{L} \mathcal{U}_{\mathfrak{q}} s \ell(2)$-action described in App. C.1 Taking the irreducible submodule $\mathrm{X}_{s_{2}, r_{2}}^{\beta}$ and subquotient $\mathrm{X}_{p-s_{2}, r_{2}+1}^{-\beta}$ of the module $\mathrm{N}_{s_{2}, r_{2}}^{\beta}(1)$, we consider the two (complementary) subspaces $\mathbf{X}_{s_{1}, r_{1}}^{\alpha} \otimes \mathbf{X}_{s_{2}, r_{2}}^{\beta}$ and $\mathbf{X}_{s_{1}, r_{1}}^{\alpha} \otimes \mathbf{X}_{p-s_{2}, r_{2}+1}^{-\beta}$ in the tensor product space with the bases $\left\{\mathrm{a}_{n^{\prime}, m^{\prime}} \otimes \mathrm{b}_{n, m}\right\}$ and $\left\{\mathrm{a}_{n^{\prime}, m^{\prime}} \otimes \mathrm{r}_{k, l}\right\}$, respectively, and decompose them using (4.1). Projectives obtained from these tensor products are direct summands because any projective $\mathcal{L} \mathcal{U}_{\mathfrak{q}} s \ell(2)$-module is also injective (the contragredient one to a projective module) and is therefore a direct summand in any module into which it is embedded. We thus obtain the decomposition

$$
\mathrm{X}_{s_{1}, r_{1}}^{\alpha} \otimes \mathrm{N}_{s_{2}, r_{2}}^{\beta}(1)=\mathbb{P} \oplus \mathbb{I},
$$

where $\mathbb{P}$ is isomorphic to the direct sum over all projective modules in the first row in (4.2) while the module $\mathbb{I}$ has the following relation in the Grothendieck ring

$$
[\mathbb{I}]=\sum_{\substack{r=\left|r_{1}-r_{2}\right|+1 \\ \text { step }=2}}^{r_{1}+r_{2}-1} \sum_{\substack{s=\left|s_{1}-s_{2}\right|+1 \\ \text { step }=2}}^{\substack{\min \left(s_{1}+s_{2}-1, 2 p-s_{1}-s_{2}-1\right)}} \mathrm{X}_{s, r}^{\alpha \beta}+\sum_{\substack{r=\left|r_{1}-r_{2}-1\right|+1 \\ \text { step }=2}}^{r_{1}+r_{2}} \sum_{\substack{s=\left|s_{1}+s_{2}-p\right|+1 \\ \text { step }=2}}^{\substack{\min \left(p+s_{1}-s_{2}-1, p-s_{1}+s_{2}-1\right)}} \mathrm{X}_{s, r}^{-\alpha \beta},
$$

where the first sum contributes to the socle $\operatorname{soc}(\mathbb{I})$ of $\mathbb{I}$ because this direct sum is the submodule in the module $X_{s_{1}, r_{1}}^{\alpha} \otimes X_{s_{2}, r_{2}}^{\beta}$ which is embedded into $X_{s_{1}, r_{1}}^{\alpha} \otimes N_{s_{2}, r_{2}}^{\beta}(1)$. We next show that $\mathbb{I}$ turns out to be a direct sum of indecomposables and the first sum in (4.5) exhausts the socle of $\mathbb{I}$, and moreover we show that the radical $\operatorname{rad}(\mathbb{I}) \cong \operatorname{soc}(\mathbb{I})$, i.e. $\operatorname{top}(\mathbb{I}) \cong \mathbb{I} / \operatorname{soc}(\mathbb{I})$ is given by the second sum in (4.5). 
We give now explicit expressions for cyclic vectors generating the module $\mathbb{I}$ in (4.4). We begin with expressions for highest weight vectors $t_{0,0}^{s, r}$ of the summands $X_{s, r}^{-\alpha \beta}$ in the second direct sum in (4.5),

$$
\mathrm{t}_{0,0}^{p+s_{1}-s_{2}-2 n-1, r_{1}+r_{2}-2 m}=\sum_{i=0}^{n} \sum_{j=0}^{m} A_{i} B_{j} \mathrm{a}_{i, j} \otimes \mathrm{r}_{n-i, m-j},
$$

where $\max \left(0, s_{1}-s_{2}\right) \leqslant n \leqslant \min \left(s_{1}, p-s_{2}\right)-1$, and $0 \leqslant m \leqslant \min \left(r_{1}, r_{2}+1\right)-1$, and the coefficients

$$
A_{i}=\left(\alpha \mathfrak{q}^{2 n+s_{2}}\right)^{i} \mathfrak{q}^{-i^{2}} \frac{([n] !)^{2}\left[s_{1}-i-1\right] !\left[p-s_{2}-n+i-1\right] !}{\left[s_{1}-n\right] !\left[p-s_{2}-n\right] ![i] ![n-i] !},
$$

and

$$
B_{j}=\left(\alpha^{p}(-1)^{s_{1}}\right)^{j} \frac{(m !)^{2}\left(r_{1}-j-1\right) !\left(r_{2}-m+j\right) !}{\left(r_{1}-m\right) !\left(r_{2}+1-m\right) ! j !(m-j) !} .
$$

The highest weight vectors $b_{0,0}^{s, r}$ of the summands $X_{s, r}^{\alpha \beta}$ in the first direct sum in (4.5) have a similar expression with the substitutions $\mathrm{r}_{n-i, m-j} \rightarrow \mathrm{b}_{n-i, m-j}, s_{2} \rightarrow p-s_{2}$ and $r_{2} \rightarrow r_{2}-1$ to be applied in (4.6).

Finally, cyclic vectors generating $\mathbb{I}$ can be taken as $t_{0,1}^{s, r} \equiv f t_{0,0}^{s, r}$ with the expression

$$
\mathrm{t}_{0,1}^{p+s_{1}-s_{2}-2 n-1, r_{1}+r_{2}-2 m}=\sum_{i=0}^{n} \sum_{j=0}^{m+1} A_{i} C_{j} \mathrm{a}_{i, j} \otimes \mathrm{r}_{n-i, m+1-j},
$$

where $\max \left(0, s_{1}-s_{2}\right) \leqslant n \leqslant \min \left(s_{1}, p-s_{2}\right)-1$, and $0 \leqslant m \leqslant \min \left(r_{1}, r_{2}+1\right)-1$.

$$
C_{j}=B_{j} \alpha^{p}(-1)^{s_{1}-1}+B_{j-1}
$$

and $B_{j}$ is defined in (4.8) and we set $B_{-1} \equiv 0$. The following relation takes then place in the module $\mathbb{I}$, or in the tensor product (4.2),

$$
E \mathrm{t}_{0,1}^{s, r} \sim \frac{r-1}{r} \mathrm{~b}_{p-s-1,1}^{p-s, r+1}+\frac{1}{r} \mathrm{~b}_{p-s-1,0}^{p-s, r-1},
$$

where $\left|s_{1}-s_{2}\right|+1 \leqslant s \leqslant \min \left(s_{1}+s_{2}, 2 p-s_{1}-s_{2}\right)-1$ and $\left|r_{1}-r_{2}\right|+1 \leqslant r \leqslant r_{1}+r_{2}-1$, and $\mathrm{b}_{n, k}^{s, r}=F^{n} f^{k} \mathrm{~b}_{0,0}^{s, r}$, with $\mathrm{b}_{0,0}^{s, r}$ defined above after (4.8). Using the definition of $\mathrm{N}_{s, r}(n)$ and $\mathrm{W}_{s, r}(n)$ given in $\mathbf{3 . 2}$ (see also an explicit example of $\mathrm{W}_{s, r}^{ \pm}(2)$ in App. C.2), we state that the module $\mathbb{I}$ is isomorphic to the third direct sum (in the second row) in (4.2). Combining with (4.4), this finishes our proof of the decomposition (4.2).

The decomposition (4.3) is obtained in a very similar way and involves the basis (C.4) in $\eta_{s_{2}, r_{2}}^{\beta}(1)$ with the action given also in App. C.1.

We now turn to a more complicated case of tensor products of two $\mathrm{N}$ - or $И$-type modules.

4.2. Theorem. For $1 \leqslant s_{1}, s_{2} \leqslant p-1, r_{1}, r_{2} \geqslant 1$, and $\alpha, \beta= \pm$, we have isomorphisms of $\mathcal{L} \mathcal{U}_{\mathfrak{q}} s \ell(2)$-modules 


$$
\begin{aligned}
& \mathrm{N}_{s_{1}, r_{1}}^{\alpha}(1) \otimes \mathrm{N}_{s_{2}, r_{2}}^{\beta}(1)=\bigoplus_{\substack{r=\left|r_{1}-r_{2}\right|+1 \\
\text { step }=2}}^{r_{1}+r_{2}-1} \bigoplus_{\substack{s=\left|s_{1}-s_{2}\right|+1 \\
\text { step=2 }}}^{p-\gamma_{2}} \mathrm{P}_{s, r}^{\alpha \beta} \oplus \bigoplus_{\substack{s=s_{1}+s_{2}+1 \\
\text { step }=2}}^{p-\gamma_{2}} \mathrm{P}_{s, r_{1}+r_{2}+1}^{\alpha \beta} \oplus \\
& \oplus \bigoplus_{\substack{r=\left|r_{1}-r_{2}+\operatorname{sg}\left(s_{2}-s_{1}\right)\right|+1 \\
\text { step }=2}}^{r_{1}+r_{2}} \bigoplus_{\substack{s=\left|s_{1}-s_{2}\right|+1 \\
\text { step }=2}}^{p-\gamma_{1}} \mathrm{P}_{s, r}^{-\alpha \beta} \oplus \bigoplus_{\substack{s=\left|p-s_{1}-s_{2}\right|+1 \\
\text { step }=2}}^{p-\left|s_{1}-s_{2}\right|-1} \mathrm{~N}_{s, r_{1}+r_{2}}^{-\alpha \beta}(1)
\end{aligned}
$$

and the tensor product of two modules contragredient to $\mathrm{N}_{s, r}^{ \pm}(1)$ is

$$
\boldsymbol{U}_{s_{1}, r_{1}}^{\alpha}(1) \otimes \boldsymbol{U}_{s_{2}, r_{2}}^{\beta}(1)=\left(\mathrm{N}_{s_{1}, r_{1}}^{\alpha}(1) \otimes \mathrm{N}_{s_{2}, r_{2}}^{\beta}(1)\right)^{*}, \quad \text { with } \mathrm{P}^{*} \equiv \mathrm{P}, \mathrm{N}^{*} \equiv И,
$$

and the tensor product of $\mathrm{N}$ - and И-type modules decomposes as

$$
\begin{aligned}
& \mathrm{N}_{s_{1}, r_{1}}^{\alpha}(1) \otimes \boldsymbol{U}_{s_{2}, r_{2}}^{\beta}(1)=\bigoplus_{\substack{r=\left|r_{1}-r_{2}\right|+2 \\
\text { step }=2}}^{r_{1}+r_{2}} \bigoplus_{\substack{s=\left|p-s_{1}-s_{2}\right|+1 \\
\text { step=2 }}}^{p-\gamma_{1}} \mathrm{P}_{s, r}^{-\alpha \beta} \oplus \bigoplus_{\substack{s=p-\left|s_{1}-s_{2}\right|+1 \\
\text { step }=2}}^{p-\gamma_{1}} \mathrm{P}_{s,\left|r_{1}-r_{2}\right|}^{-\alpha \beta} \delta_{\mathrm{sg}\left(r_{1}-r_{2}\right), \operatorname{sg}\left(s_{1}-s_{2}\right)} \oplus
\end{aligned}
$$

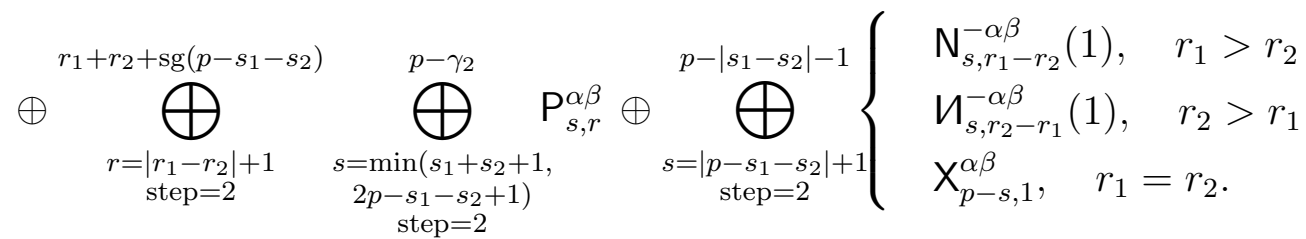

Proof. We consider first the tensor product (4.12). The first and the second tensorands have the bases $\left\{\mathrm{b}_{n, m}^{1}\right\} \cup\left\{\mathrm{r}_{k, l}^{1}\right\}$ and $\left\{\mathrm{b}_{n, m}^{2}\right\} \cup\left\{\mathrm{r}_{k, l}^{2}\right\}$ respectively, see (C.3), with the $\mathcal{L} \mathcal{U}_{\mathfrak{q}} s \ell(2)$-action described in App. C.1. Taking the irreducible submodules $\mathrm{X}_{s_{1}, r_{1}}^{\alpha}$ and $\mathrm{X}_{s_{2}, r_{2}}^{\beta}$ and subquotients $\mathbf{X}_{p-s_{1}, r_{1}+1}^{-\alpha}$ and $\mathbf{X}_{p-s_{2}, r_{2}+1}^{-\beta}$ of the modules, we consider the four subspaces $\mathbf{X}_{s_{1}, r_{1}}^{\alpha} \otimes \mathbf{X}_{s_{2}, r_{2}}^{\beta}$, $\mathbf{X}_{s_{1}, r_{1}}^{\alpha} \otimes \mathbf{X}_{p-s_{2}, r_{2}+1}^{-\beta}, \mathbf{X}_{p-s_{1}, r_{1}+1}^{-\alpha} \otimes \mathbf{X}_{s_{2}, r_{2}}^{\beta}$, and $\mathbf{X}_{p-s_{1}, r_{1}+1}^{-\alpha} \otimes \mathbf{X}_{p-s_{2}, r_{2}+1}^{-\beta}$ in the tensor product space with the bases $\left\{\mathrm{b}_{n, m}^{1} \otimes \mathrm{b}_{k, l}^{2}\right\},\left\{\mathrm{b}_{n, m}^{1} \otimes \mathrm{r}_{k, l}^{2}\right\},\left\{\mathrm{r}_{k, l}^{1} \otimes \mathrm{b}_{n, m}^{2}\right\}$, and $\left\{\mathrm{r}_{n, m}^{1} \otimes \mathrm{r}_{k, l}^{2}\right\}$, respectively, and decompose them using (4.1). Projective modules obtained from these tensor products are direct summands because they are also injective. Irreducible modules obtained from these tensor products contribute to submodules or subquotients in indecomposable direct summands. We thus obtain the decomposition

$$
\mathrm{N}_{s_{1}, r_{1}}^{\alpha}(1) \otimes \mathrm{N}_{s_{2}, r_{2}}^{\beta}(1)=\mathbb{P} \oplus \mathbb{I},
$$

where $\mathbb{P}$ is isomorphic to the direct sums

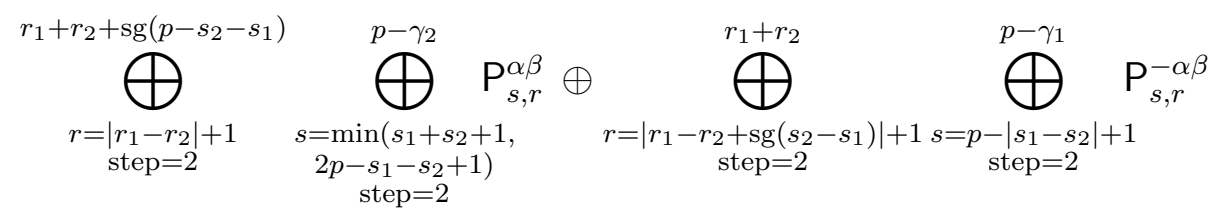

over projective modules (not all) in 4.12 ) while the module $\mathbb{I}$ has the following relation in the Grothendieck ring 


$$
\begin{aligned}
& {[\mathbb{I}]=\sum_{\substack{r=\left|r_{1}-r_{2}\right|+1 \\
\text { step }=2}}^{r_{1}+r_{2}-1} \sum_{\substack{s=\left|s_{1}-s_{2}\right|+1 \\
\text { step }=2}}^{\substack{\min \left(s_{1}+s_{2}-1, 2 p-s_{1}-s_{2}-1\right)}} \mathrm{X}_{s, r}^{\alpha \beta}+\sum_{\substack{r=\left|r_{1}-r_{2}\right|+1 \\
\text { step=2 }}}^{r_{1}+r_{2}+1} \sum_{\substack{s=\left|s_{1}-s_{2}\right|+1 \\
\text { step }=2}}^{\substack{\min \left(s_{1}+s_{2}-1 \\
2 p-s_{1}-s_{2}-1\right)}} \mathrm{X}_{s, r}^{\alpha \beta}} \\
& +\sum_{\substack{r=\left|r_{1}-r_{2}-1\right|+1 \\
\text { step }=2}}^{r_{1}+r_{2}} \sum_{\substack{s=\left|s_{1}+s_{2}-p\right|+1 \\
\text { step }=2}}^{\substack{\min \left(p+s_{1}-s_{2}-1, p-s_{1}+s_{2}-1\right)}} \mathrm{X}_{s, r}^{-\alpha \beta}+\sum_{\substack{r=\left|r_{1}-r_{2}+1\right|+1 \\
\text { step }=2}}^{r_{1}+r_{2}} \sum_{\substack{s=\left|s_{1}+s_{2}-p\right|+1 \\
\text { step }=2}}^{\substack{\min \left(p-s_{1}+s_{2}-1, p+s_{1}-s_{2}-1\right)}} \mathrm{X}_{s, r}^{-\alpha \beta}
\end{aligned}
$$

where the first sum obviously contributes to the $\operatorname{socle} \operatorname{soc}(\mathbb{I})$ of $\mathbb{I}$ because this direct sum is the submodule in the module $\mathbf{X}_{s_{1}, r_{1}}^{\alpha} \otimes \mathbf{X}_{s_{2}, r_{2}}^{\beta}$ which is embedded into $\mathbf{N}_{s_{2}, r_{2}}^{\beta}(1) \otimes \mathbf{N}_{s_{2}, r_{2}}^{\beta}(1)$. We next show that II turns out to be a direct sum of indecomposables and the first sum in (4.16) exhausts the socle (the first level) of $\mathbb{I}$, and moreover we show below that the second level consists of the two last sums (in the second row) while the third level of $\mathbb{I}$ is given by the second sum in the first row of (4.16).

We note that if the summands contribute to a subquotient in an indecomposable module of semisimple length not greater than 2 (for example to $\mathrm{N}_{s, r}(n)$ ) then the Casimir element (2.6) has a diagonal form on it. If the summands correspond to top subquotients in projective modules then the Casimir element is non-diagonalizable on them. Thus, the structure of the tensor product can be studied by diagonalizability of the Casimir element

$$
\boldsymbol{C}=\left(\mathfrak{q}-\mathfrak{q}^{-1}\right)^{2} E F+\mathfrak{q}^{-1} K+\mathfrak{q} K^{-1} .
$$

Our next strategy consists of two steps: (1) to study a Jordan cell decomposition of the matrix representig the Casimir element obtaining thus a projective module summand $\mathbb{P}_{1}$ in $\mathbb{I}$ and then (2) to give cyclic vectors generating direct summands in $\mathbb{I}$ which have the semisimple length not greater than 2 .

We assume in what follows that

$$
0 \leqslant n \leqslant \min \left(p-s_{1}, p-s_{2}\right)-1, \quad 0 \leqslant m_{1} \leqslant r_{1}+1, \quad 0 \leqslant m_{2} \leqslant r_{2}+1 .
$$

For any triplet $\left(n, m_{1}, m_{2}\right)$ with values from (4.17), let $T^{n}$ denotes a $(n+1) \times(n+1)$-matrix representing the action of $\boldsymbol{C}$ restricted to the subspace spanned by $\left\{\mathrm{r}_{i, m_{1}}^{1} \otimes \mathrm{r}_{n-i, m_{2}}^{2}\right\}$, where $0 \leqslant i \leqslant n$ and we omit $m_{1}$ and $m_{2}$ indexes in the notation for this matrix, i.e. we consider a decomposition (of the representation of) $\boldsymbol{C}=\boldsymbol{C}^{(d)}+\boldsymbol{C}^{(n)}$ with a diagonalizable part $\boldsymbol{C}^{(d)}=T^{n}$ while a non-diagonalizable part $\boldsymbol{C}^{(n)}$ will be given below. $T^{n}$ is a three-diagonal 
matrix with the elements

$$
\begin{aligned}
& T_{i, i-1}^{n}=\left(\mathfrak{q}-\mathfrak{q}^{-1}\right)^{2} \bar{\beta}[\bar{i}]\left[\bar{s}_{1}-\bar{i}\right] \mathfrak{q}^{\bar{s}_{2}-\bar{s}_{1}-2 \bar{n}+4 \bar{i}+2}, \\
& T_{i, i}^{n}=\bar{\alpha} \bar{\beta}\left(\mathfrak{q}^{-\bar{s}_{1}+2 \bar{i}+1}\left(\mathfrak{q}^{\bar{s}_{2}}+\mathfrak{q}^{-\bar{s}_{2}}\right)+\mathfrak{q}^{\bar{s}_{2}-2 \bar{n}+2 \bar{i}-1}\left(\mathfrak{q}^{\bar{s}_{1}}+\mathfrak{q}^{-\bar{s}_{1}}\right)-\left(\mathfrak{q}+\mathfrak{q}^{-1}\right) \mathfrak{q}^{\bar{s}_{2}-\bar{s}_{1}-2 \bar{n}+4 \bar{i}}\right), \\
& T_{i, i+1}^{n}=\left(\mathfrak{q}-\mathfrak{q}^{-1}\right)^{2} \bar{\beta}[\bar{n}-\bar{i}]\left[\bar{s}_{2}-\bar{n}-\bar{i}\right], \\
& T_{i, i \pm k}^{n}=0, \quad k \geqslant 2 .
\end{aligned}
$$

where $\bar{s}_{1}=p-s_{1}, \bar{s}_{2}=p-s_{2}, \bar{n}=n, \bar{\alpha}=-\alpha, \bar{\beta}=-\beta$, and $\bar{i}=i$.

The subspace $\left\{\mathrm{r}_{i, m_{1}}^{1} \otimes \mathrm{r}_{n-i, m_{2}}^{2}\right\}$ is not invariant with respect to the Casimir element action. We now describe the non-diagonalizable part $C^{(n)}$. Depending on parameters, there exist two cases:

(1) When $s_{1}+s_{2}-p+n<0$, the smallest invariant subspace including $\left\{\mathrm{r}_{i, m_{1}}^{1} \otimes \mathrm{r}_{n-i, m_{2}}^{2}\right\}$ is $V^{n}=\left\{\mathrm{r}_{i, m_{1}}^{1} \otimes \mathrm{r}_{n-i, m_{2}}^{2}\right\} \cup\left\{\mathrm{r}_{j, m_{1}}^{1} \otimes \mathrm{b}_{s_{2}+n-j, m_{2}-1}^{2}\right\} \cup\left\{\mathrm{b}_{k, m_{1}-1}^{1} \otimes \mathrm{r}_{s_{1}+n-k, m_{2}}^{2}\right\}$, where $n+1 \leqslant j \leqslant s_{2}+n$ and $0 \leqslant k \leqslant s_{1}-1$. Let $C_{1}^{n}$ denotes a $s_{2} \times s_{2}$ matrix representing $C$ within the subspace $\left\{\mathrm{r}_{j, m_{1}}^{1} \otimes \mathrm{b}_{s_{2}+n-j, m_{2}-1}^{2}\right\}$ and $C_{2}^{n}-\mathrm{a} s_{1} \times s_{1}$ matrix representing $C$ within $\left\{\mathrm{b}_{k, m_{1}-1}^{1} \otimes \mathrm{r}_{s_{1}+n-k, m_{2}}^{2}\right\}$. They have the elements as in (4.18) with the substitutions $\bar{s}_{1}=p-s_{1}, \bar{s}_{2}=s_{2}, \bar{n}=p-s_{2}+n, \bar{i}=j, \bar{\alpha}=-\alpha, \bar{\beta}=\beta$ and $\bar{s}_{1}=s_{1}, \bar{s}_{2}=p-s_{2}, \bar{n}=p-s_{1}+n, \bar{i}=k, \bar{\alpha}=\alpha, \bar{\beta}=-\beta$ respectively. A matrix representing the Casimir element within the whole invariant subspace $V^{n}$ then takes the form

$$
\begin{aligned}
& \begin{array}{lll}
T^{n} & 0 & 0
\end{array} \\
& {\left[0^{u_{1}}\right] \quad C_{1}^{n} \quad 0} \\
& {\left[{ }_{u_{2}} 0\right] \quad 0 \quad C_{2}^{n}}
\end{aligned}
$$

where $\left[0^{u}\right]$ denotes a matrix with all elements equal zero except for the right top one which equals

$$
u_{1}=-\left(\mathfrak{q}-\mathfrak{q}^{-1}\right)^{2} \beta m_{2} \frac{(-1)^{p}\left[s_{2}\right]}{[p-1] !},
$$

and $\left[v_{v} 0\right]$ denotes a matrix with all elements equal zero except for the left bottom one which equals

$$
u_{2}=-\left(\mathfrak{q}-\mathfrak{q}^{-1}\right)^{2} \beta m_{1} \frac{(-1)^{p}\left[s_{1}\right]}{[p-1] !} \mathfrak{q}^{s_{1}-s_{2}-2 n+2} .
$$

(2) When $s_{1}+s_{2}-p+n \geqslant 0$, let $C_{1}^{n}$ similarly denotes a matrix representing the Casimir element $C$ within the subspace $\left\{\mathrm{r}_{j, m_{1}}^{1} \otimes \mathrm{b}_{s_{2}+n-j, m_{2}-1}^{2}\right\}$ and $C_{2}^{n}-$ a matrix representing $\boldsymbol{C}$ within $\left\{\mathrm{b}_{k, m_{1}-1}^{1} \otimes \mathrm{r}_{s_{1}+n-k, m_{2}}^{2}\right\}$, where $n+1 \leqslant j \leqslant p-s_{1}-1$ and $s_{2}+s_{1}-p+$ $n+1 \leqslant k \leqslant s_{1}-1$. They have the elements as in (4.18) with $\bar{s}_{1}=p-s_{1}, \bar{s}_{2}=s_{2}$, 
$\bar{n}=p-s_{2}+n, \bar{i}=j, \bar{\alpha}=-\alpha, \bar{\beta}=\beta$ and $\bar{s}_{1}=s_{1}, \bar{s}_{2}=p-s_{2}, \bar{n}=p-s_{1}+n, \bar{i}=k$, $\bar{\alpha}=\alpha, \bar{\beta}=-\beta$, respectively. Let $B_{1}^{n}$ and $B_{2}^{n}$ also denotes matrices representing $C$ within $\left\{\mathrm{b}_{m, m_{1}}^{1} \otimes \mathrm{b}_{s_{2}+s_{1}-p+n-m, m_{2}-1}^{2}\right\}$ and $\left\{\mathrm{b}_{m, m_{1}-1}^{1} \otimes \mathrm{b}_{s_{2}+s_{1}-p+n-m, m_{2}}^{2}\right\}$, respectively, where $0 \leqslant m \leqslant s_{1}+s_{2}-p+n$. They have also the elements (4.18) with $\bar{s}_{1}=s_{1}$, $\bar{s}_{2}=s_{2}, \bar{n}=s_{1}+s_{2}-p+n, \bar{\alpha}=\alpha, \bar{\beta}=\beta$ and $\bar{i}=m$. The matrix representing $C$ within the invariant subspace $V^{n}=\left\{\mathrm{r}_{i, m_{1}}^{1} \otimes \mathrm{r}_{n-i, m_{2}}^{2}\right\} \cup\left\{\mathrm{r}_{j, m_{1}}^{1} \otimes \mathrm{b}_{s_{2}+n-j, m_{2}-1}^{2}\right\} \cup$ $\left\{\mathrm{b}_{k, m_{1}-1}^{1} \otimes \mathrm{r}_{s_{1}+n-k, m_{2}}^{2}\right\} \cup\left\{\mathrm{b}_{m, m_{1}}^{1} \otimes \mathrm{b}_{s_{2}+s_{1}-p+n-m, m_{2}-1}^{2}\right\} \cup\left\{\mathrm{b}_{m, m_{1}-1}^{1} \otimes \mathrm{b}_{s_{2}+s_{1}-p+n-m, m_{2}}^{2}\right\}$ takes then the block-structure

$$
\begin{aligned}
& \begin{array}{lllll}
T^{n} & 0 & 0 & 0 & 0
\end{array} \\
& {\left[0^{u_{1}}\right] \quad C_{1}^{n} \quad 0 \quad 000} \\
& A^{n}=\quad 0 \quad\left[0^{v_{1}}\right] \quad B_{1}^{n} \quad 0 \quad 0 \\
& \begin{array}{llll}
0 & 0 & 0 & B_{2}^{n}
\end{array}\left[v_{2} 0\right] \\
& {\left[u_{2} 0\right] \quad 0 \quad 0 \quad 0 \quad C_{2}^{n}}
\end{aligned}
$$

where we set $v_{1}=\left(\mathfrak{q}-\mathfrak{q}^{-1}\right)^{2} \beta\left[p-s_{1}-n-1\right]\left[s_{1}+s_{2}-p+n+1\right]$ and $v_{2}=$ $\left(\mathfrak{q}-\mathfrak{q}^{-1}\right)^{2} \beta\left[p-s_{2}-n-1\right]\left[s_{1}+s_{2}-p+n+1\right] \mathfrak{q}^{s_{1}+s_{2}-p+2 n}$, and $u_{1}, u_{2}$ are given in (4.20) and (4.21).

Thus, we have a set of subspaces $V^{n}$ which are invariant with respect to the Casimir element action in the whole space of the tensor product.

Next, we assume a decomposition

$$
\mathbb{I}=\mathbb{P}_{1} \oplus \mathbb{I}_{1},
$$

where $\mathbb{P}_{1}$ is a maximum projective submodule, i.e. the direct sum over all projective covers embedded in $\mathbb{I}$, while $\mathbb{I}_{1}$ is a module of the semisimple length not greater than 2 . We should note that

$$
\left[\operatorname{top}\left(\mathbb{P}_{1}\right)\right] \subset \sum_{\substack{r=\left|r_{1}-r_{2}\right|+1 \\ \text { step }=2}}^{r_{1}+r_{2}+1} \sum_{\substack{s=\left|s_{1}-s_{2}\right|+1 \\ \text { step }=2}}^{\substack{\min \left(s_{1}+s_{2}-1, 2 p-s_{1}-s_{2}-1\right)}} \mathrm{X}_{s, r}^{\alpha \beta},
$$

where the sum was introduced in (4.16), and every highest weight vector from the sum (rigorously, in the corresponding direct sum of modules considered as a quotient of $\mathbb{I}$, of course) appears in the direct sum of spaces $\bigoplus_{n} V^{n}$. Therefore, each projective module that appears in $\mathbb{P}_{1}$ should contribute to a set of Jordan cells (of rank 2) in the matrix of $\boldsymbol{C}$ on the space $\bigoplus_{n} V^{n}$. We thus need to determine the Jordan cells structure on all subspaces $V^{n}$.

When $s_{1}+s_{2}>p$, the Casimir element matrix has the block-structure $A^{n}$ (4.22) for all subspaces $V^{n}$, with $0 \leqslant n \leqslant \min \left(p-s_{1}, p-s_{2}\right)-1$. The set of eigenvalues of the $A^{n}$ matrix 
coinsides with the union of the sets of eigenvalues of the blocks $T^{n}, C_{1}^{n}, C_{2}^{n}, B_{1}^{n}$ and $B_{2}^{n}$. The eigenvalues of these matrices are

$$
\begin{array}{rr}
T^{n}: & \left\{\mathfrak{q}^{2 p-s_{1}-s_{2}-2 k-1}+\mathfrak{q}^{-\left(2 p-s_{1}-s_{2}-2 k-1\right)}, 0 \leqslant k \leqslant n\right\}, \\
C_{1}^{n}: & \left\{-\mathfrak{q}^{s_{2}+p-s_{1}-2 k-1}-\mathfrak{q}^{-\left(s_{2}+p-s_{1}-2 k-1\right)}, 0 \leqslant k \leqslant p-s_{1}-n-2\right\}, \\
C_{2}^{n}: & \left\{-\mathfrak{q}^{s_{1}+p-s_{2}-2 k-1}-\mathfrak{q}^{-\left(s_{1}+p-s_{2}-2 k-1\right)}, 0 \leqslant k \leqslant p-s_{2}-n-2\right\}, \\
B_{1}^{n}: & \left\{\mathfrak{q}^{s_{1}+s_{2}-2 k-1}+\mathfrak{q}^{-\left(s_{1}+s_{2}-2 k-1\right)}, 0 \leqslant k \leqslant n+s_{1}+s_{2}-p\right\}, \\
B_{2}^{n}: & \left\{\mathfrak{q}^{s_{1}+s_{2}-2 k-1}+\mathfrak{q}^{-\left(s_{1}+s_{2}-2 k-1\right)}, 0 \leqslant k \leqslant n+s_{1}+s_{2}-p\right\} .
\end{array}
$$

We see that the eigenvalues

$$
\left\{\mathfrak{q}^{2 p-s_{1}-s_{2}-2 k-1}+\mathfrak{q}^{-\left(2 p-s_{1}-s_{2}-2 k-1\right)}, 0 \leqslant k \leqslant n\right\}
$$

are degenerated (see the first and two last rows above). Whether there are three eigenvectors corresponding to each triplet of the degenerated eigenvalues or only two of them the Casimir element is diagonalizable or not and the corresponding irreducible term is a subquotient in a non-projective module or in a projective one. We should note that there are other degenerated eigenvalues - eigenvalues from the second and third rows which partially coincide, namely the range $\left\{-\mathfrak{q}^{p-\left|s_{1}-s_{2}\right|-2 k-1}-\mathfrak{q}^{-\left(p-\left|s_{1}-s_{2}\right|-2 k-1\right)}, 0 \leqslant k \leqslant p-\max \left(s_{1}, s_{2}\right)-n-2\right\}$, but the corresponding subspaces are direct summands as modules over the subalgebra generated by $\boldsymbol{C}$, therefore these eigenvalues can not correspond to Jordan cells. The degenerated eigenvalues from the last two rows (see the fourth and fifth row above) from the range $\left\{\mathfrak{q}^{s_{1}+s_{2}-2 k-1}+\mathfrak{q}^{-\left(s_{1}+s_{2}-2 k-1\right)}, 0 \leqslant k \leqslant s_{1}+s_{2}-p-1\right\}$ comlementary to the range (4.25) correspond to non-trivial Jordan cells but these cells contribute to projective modules that are direct summands in the submodule $X_{s_{1}, r_{1}}^{\alpha} \otimes \mathbf{X}_{s_{2}, r_{2}}^{\beta}$ of the tensor-product module and they are therefore submodules in $\mathbb{P}$ and not in $\mathbb{I}$ (see (4.4)).

We analyze next the most degenerate range corresponding to 4.25). We claim that there are only two eigenvectors for each eigenvalue from the set (4.25): one vector is from the subspace $\left\{\mathrm{b}_{m, m_{1}}^{1} \otimes \mathrm{b}_{s_{2}+s_{1}-p+n-m, m_{2}-1}^{2}\right\}$ and it coincides with the corresponding eigenvector of the matrix $B_{1}^{n}$, and the second one is from $\left\{\mathrm{b}_{m, m_{1}-1}^{1} \otimes \mathrm{b}_{s_{2}+s_{1}-p+n-m, m_{2}}^{2}\right\}$ and coincides with the eigenvector of the matrix $B_{2}^{n}$, where $0 \leqslant m \leqslant s_{1}+s_{2}-p+n$, and consequently there is a Jordan cell contributing to projective modules in $\mathbb{I}$. To prove that a third linearly independent eigenvector does not exist, we consider the equation $\left(A^{n}-\lambda I\right) \mathrm{v}=0$ (see (4.22) $)$, with $\lambda$ being an eigenvalue from the set (4.25). Since there exist at least two solutions of this equation, we can decrease the number of variables by two. The remaining equation however has no a solution. A similar analysis can be repeated for the case $p-n \leqslant s_{1}+s_{2} \leqslant p$.

For $s_{1}+s_{2}<p-n$, the only difference from the previous cases is that the matrix $T^{n}$ is not diagonalizable which lead to projective modules contributing to $\mathbb{P}$ while the module II has no projective modules as direct summands because (1) the socle of a projective direct summand in $\mathbb{I}$ should be a proper subspace in the submodule $X_{s_{1}, r_{1}}^{\alpha} \otimes \mathbf{X}_{s_{2}, r_{2}}^{\beta}$, which is spanned 
by vectors of the type $b \otimes b$, but (2) all Jordan cells of rank 2 in the Jordan form of the $A^{n}$ matrix given in (4.19) are spanned by vectors which have an empty intersection with the submodule $\mathrm{X}_{s_{1}, r_{1}}^{\alpha} \otimes \mathrm{X}_{s_{2}, r_{2}}^{\beta}$ of the tensor-product module.

Combining with the $s \ell(2)$ content of $\mathbb{I}$ in (4.16), we finally get that the set of Jordan cells corresponds to the decomposition (4.23) where

$$
\mathbb{P}_{1}=\bigoplus_{\substack{r=\left|r_{1}-r_{2}\right|+1 \\ \text { step }=2}}^{r_{1}+r_{2}-1} \bigoplus_{\substack{s=\left|s_{1}-s_{2}\right|+1 \\ \text { step }=2}}^{\substack{\min \left(s_{1}+s_{2}-1 \\ 2 p-s_{1}-s_{2}-1\right)}} \mathrm{P}_{s, r}^{\alpha \beta}
$$

while the module $\mathbb{I}_{1}$ has the following relation in the Grothendieck ring

$$
\left[\mathbb{I}_{1}\right]=\sum_{\substack{s=\left|p-s_{1}-s_{2}\right|+1 \\ \text { step }=2}}^{p-\left|s_{1}-s_{2}\right|-1} \mathrm{X}_{s, r_{1}+r_{2}}^{-\alpha \beta}(1)+\sum_{\substack{s=\left|s_{1}-s_{2}\right|+1 \\ \text { step=2 }}}^{\substack{\min \left(s_{1}+s_{2}-1, 2 p-s_{1}-s_{2}-1\right)}} \mathrm{X}_{s, r_{1}+r_{2}+1}^{\alpha \beta}(1) .
$$

Similarly to the proof in 4.1, we obtain

$$
\mathbb{I}_{1}=\bigoplus_{\substack{s=\left|p-s_{1}-s_{2}\right|+1 \\ \text { step }=2}}^{p-\left|s_{1}-s_{2}\right|-1} \mathrm{~N}_{s, r_{1}+r_{2}}^{-\alpha \beta}(1),
$$

where highest weight vectors and cyclic vectors of the subquotients have the expressions

$$
\begin{aligned}
& \mathrm{t}_{0,0}^{2 p-s_{1}-s_{2}-2 n-1}=\sum_{i=0}^{n} A_{i} \mathrm{r}_{i, 0}^{1} \otimes \mathrm{r}_{n-i, 0}^{2}, \\
& \mathrm{t}_{0,1}^{2 p-s_{1}-s_{2}-2 n-1}=\sum_{i=0}^{n} A_{i}\left((-\alpha)^{p}(-1)^{s_{1}-1} \mathrm{r}_{i, 0}^{1} \otimes \mathrm{r}_{n-i, 1}^{2}+\mathrm{r}_{i, 1}^{1} \otimes \mathrm{r}_{n-i, 0}^{2}\right),
\end{aligned}
$$

where $\max \left(0, p-s_{1}-s_{2}\right) \leqslant n \leqslant \min \left(p-s_{1}, p-s_{2}\right)-1$, and the coefficients

$$
A_{i}=\left(\alpha \mathfrak{q}^{2 n+s_{2}}\right)^{i} \mathfrak{q}^{-i^{2}} \frac{([n] !)^{2}\left[p-s_{1}-i-1\right] !\left[p-s_{2}-n+i-1\right] !}{\left[p-s_{1}-n\right] !\left[p-s_{2}-n\right] ![i] ![n-i] !} .
$$

Since $E \mathrm{t}_{0,0}^{2 p-s_{1}-s_{2}-2 n-1}=0$ and

$$
E \mathrm{t}_{0,1}^{2 p-s_{1}-s_{2}-2 n-1} \in \bigoplus_{\substack{s=\left|p-s_{1}-s_{2}\right|+1 \\ \text { step }=2}}^{p-\left|s_{1}-s_{2}\right|-1} \mathrm{X}_{s, r_{1}+r_{2}}^{-\alpha \beta}(1)
$$

then $\mathrm{t}_{0,1}^{2 p-s_{1}-s_{2}-2 n-1}$ is a cyclic vector of $\mathrm{N}(1)$ module (see App.C.1).

Finally, a decomposition of the whole tensor product is

$$
\mathrm{N}_{s_{1}, r_{1}}^{\alpha}(1) \otimes \mathrm{N}_{s_{2}, r_{2}}^{\beta}(1)=\bigoplus_{\substack{r=\left|r_{1}-r_{2}\right|+1 \\ \text { step }=2}}^{r_{1}+r_{2}-1} \bigoplus_{\substack{s=\left|s_{1}-s_{2}\right|+1 \\ \text { step=2 }}}^{\substack{\min \left(s_{1}+s_{2}-1, 2 p-s_{1}-s_{2}-1\right)}} \mathrm{P}_{\substack{s, r \\ s=\left|p-s_{1}-s_{2}\right|+1 \\ \text { step }=2}}^{\alpha \beta} \mathrm{N}_{s, r_{1}+r_{2}}^{-\alpha \beta}(1) \oplus
$$




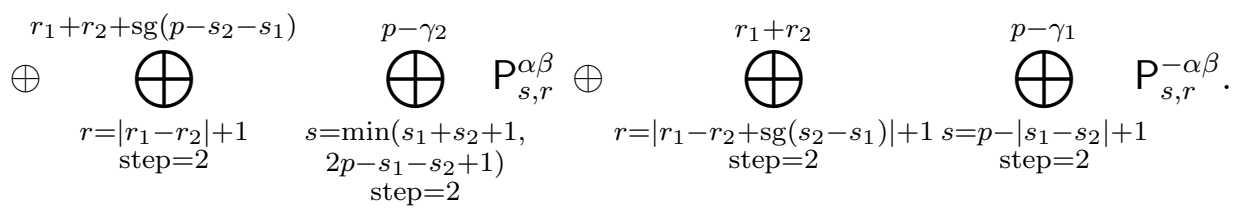

which can be rewritten as in (4.12). The decompositions (4.13) and (4.14) are obtained in a very similar way and we omit it.

The above results allow us to decompose the tensor product of an arbitrary pair of indecomposable modules over $\mathcal{L} \mathcal{U}_{\mathfrak{q}} s \ell(2)$.

4.3. Theorem. Tensor product of arbitrary two indecomposable $\mathcal{L U}_{\mathfrak{q}} s \ell(2)$-modules is obtained from the base tensor products in 4.1 and 4.2 and the following list of rules:

(1) the tensor product of $\mathrm{P}_{s, r}$ with an indecomposable module is isomorphic to the tensor product of $\mathrm{P}_{s, r}$ with the direct sum of all irreducible subquotients constituting the indecomposable module.

(2) An indecomposable module with the semisimple length 2 is the tensor product of irreducible and simplest indecomposable modules for $s=1, \ldots p-1$ and $r, n \in \mathbb{N}$ :

$$
\begin{aligned}
\mathrm{N}_{s, r}^{\alpha}(n) & =\mathrm{X}_{1, n}^{+} \otimes \mathrm{N}_{s, r+n-1}^{\alpha}(1), & \eta_{s, r}^{\alpha}(n) & =\mathrm{X}_{1, n}^{+} \otimes \boldsymbol{U}_{s, r+n-1}^{\alpha}(1), \\
\mathrm{W}_{s, r}^{\alpha}(n) & =\mathrm{X}_{1, r+n}^{-} \otimes \mathrm{N}_{p-s, n}^{\alpha}(1), & \mathrm{M}_{s, r}^{\alpha}(n) & =\mathrm{X}_{1, r+n}^{-} \otimes{И_{p-s, n}^{\alpha}(1) .}^{\alpha},
\end{aligned}
$$

Proof. We note first that the tensor product of a projective module with any indecomposable one must contain only projective modules. The projectives obtained from this tensor product are direct summands because any projective $\mathcal{L} \mathcal{U}_{\mathfrak{q}} s \ell(2)$-module is also injective (the contragredient one to a projective module) and is therefore a direct summand in any module into which it is embedded. This proves the first statement.

The second statement easily follows from the classification theorem 3.4 and Thm. 4.1

This completes the description of the tensor structure on $\mathcal{C}_{p}$. Using the associativity and commutativity of the tensor product decomposition for $\mathcal{L} \mathcal{U}_{\mathfrak{q}} s \ell(2)$, we give an exhaustive list of tensor products in App. $\mathbf{E}$

4.4. Generators. We give finally a set of generators in the tensor category $\mathcal{C}_{p}$ :

$$
\mathrm{X}_{1,1}^{ \pm}, \quad \mathrm{X}_{1,2}^{+}, \quad \mathrm{X}_{2,1}^{+}, \quad \mathrm{N}_{1,1}^{+}(1), \quad \boldsymbol{И}_{1,1}^{+}(1) .
$$

That these objects generate the tensor category $\mathcal{C}_{p}$ by successive application of the tensor product follows easily from the previous three theorems given above.

Since the construction of the tensor category $\mathcal{C}_{p}$ is complete, we can confirm a conclusion obtained in [27] that the full subcategory

$$
\mathcal{C}_{p}^{+}=\bigoplus_{s=1}^{p-1} \mathcal{C}^{+}(s) \oplus \bigoplus_{\text {odd } r \geqslant 1} \mathcal{S}^{+}(r) \oplus \bigoplus_{\text {even } r \geqslant 2} \mathcal{S}^{-}(r)
$$


in the category $\mathfrak{C}_{p}=\mathfrak{C}_{p}^{+} \oplus \mathcal{C}_{p}^{-}$is closed under the tensor product operation. All the base tensor products in $\mathrm{C}_{p}^{+}$are collected in Thm. 1.2 where we use the notation $\mathrm{X}_{s, r} \equiv \mathrm{X}_{s, r}^{\alpha(r)}$, with the sign $\alpha(r)=(-1)^{r-1}$, and similar notations for all indecomposable modules.

\section{CONCLUSION}

We have established, in 1.1, that each indecomposable $\mathcal{L} \mathcal{U}_{\mathfrak{q}} s \ell(2)$-module from the category $\mathrm{C}_{p}^{+}$has an indecomposable counterpart in the logarithmic $\mathcal{L} \mathcal{M}(1, p)$ model, for any integer $p \geqslant 2$, which can be described as a category $\mathcal{D}_{p}$ of representations of the vertex operator algebra $\mathcal{V}_{p}$ corresponding to a quotient of the universal enveloping of the Virasoro algebra with the central charge $c_{1, p}=13-6 / p-6 p$. This means that there exists a functor between the two categories $\mathcal{F}: \mathfrak{C}_{p}^{+} \rightarrow \mathcal{D}_{p}$. Moreover, by direct comparison with [49], we see that $\mathcal{F}$ is a tensor functor. This remarkable result allows us to conjecture that $\mathrm{C}_{p}^{+}$and $\mathcal{D}_{p}$ are equivalent as tensor categories. A possible way to prove the conjecture is to be reduced to a check that the category $\mathcal{D}_{p}$ contains no more indecomposable objects than $\mathcal{C}_{p}^{+}$. This can be prooved by explicit comparison of the Ext ${ }^{\bullet}$ algebras for $\mathcal{C}_{p}^{+}$and $\mathcal{D}_{p}$.

We give finally several comments on relations between extension groups for both tensor categories. For each subcategory $\mathrm{C}^{+}(s)$ with $1 \leqslant s \leqslant p-1$, the basic fact is that the space $\operatorname{Ext}_{\mathcal{L} \mathfrak{U}_{q}}^{n}$ of $n$-extensions between the irreducible modules is at most one-dimensional (see 3.1.1). We choose bases $\left\{x_{r, i}^{+}\right\}$and $\left\{x_{r, i}^{-}\right\}$, with $i \in\{0,1\}$ and $r \geqslant 1$, in the respective spaces $\mathbb{C}=\operatorname{Ext}_{\mathcal{L} \mathfrak{u}_{q}}^{1}\left(\mathrm{X}_{s, 2 r-1}^{+}, \mathrm{X}_{p-s, 2(r-i)}^{-}\right)$and $\mathbb{C}=\operatorname{Ext}_{\mathcal{L} u_{q}}^{1}\left(\mathrm{X}_{p-s, 2 r}^{-}, \mathrm{X}_{s, 2(r-i)+1}^{+}\right)$, where we set $x_{1,1}^{+} \equiv 0$. Next, the vector space

$$
\mathrm{Ext}_{s}^{\bullet}=\bigoplus_{n \geqslant 0} \bigoplus_{r, r^{\prime} \geqslant 1} \operatorname{Ext}_{\mathcal{L} \mathcal{L}_{q}}^{n}\left(\mathrm{X}_{s, 2 r-1}^{+} \oplus \mathrm{X}_{p-s, 2 r}^{-}, \mathrm{X}_{s, 2 r^{\prime}-1}^{+} \oplus \mathrm{X}_{p-s, 2 r^{\prime}}^{-}\right)
$$

is an associative algebra with respect to the Yoneda product. We propose the algebraic structure of Ext $_{s}$.

5.1. Conjecture. The algebra Ext $_{s}^{\bullet}$ is generated by $x_{r, i}^{ \pm}$with the defining relations

$$
\begin{aligned}
x_{r, i}^{+} x_{r^{\prime}, j}^{+} & =x_{r, i}^{-} x_{r^{\prime}, j}^{-}=x_{1,1}^{-} x_{1,0}^{+}=0 \\
x_{r, 0}^{+} x_{r, 1}^{-}+x_{r+1,1}^{+} x_{r, 0}^{-} & =0, \quad x_{r, 0}^{-} x_{r+1,1}^{+}+x_{r+1,1}^{-} x_{r+1,0}^{+}=0,
\end{aligned}
$$

where $i, j \in\{0,1\}$ and $r, r^{\prime} \geqslant 1$.

Let Ext ${ }^{\bullet}=\bigoplus_{s=1}^{p-1}$ Ext $_{s}^{\bullet}$. We note then that the derived category of representations of the algebra Ext ${ }^{\bullet}$ is equivalent to the derived category of $\mathcal{C}_{p}^{+}$and conjecturally to the derived category of $\mathcal{D}_{p}$. An explicit calculation of the algebra of Ext"s for the category $\mathcal{D}_{p}$ is a very important problem, which is waiting for its solution. 


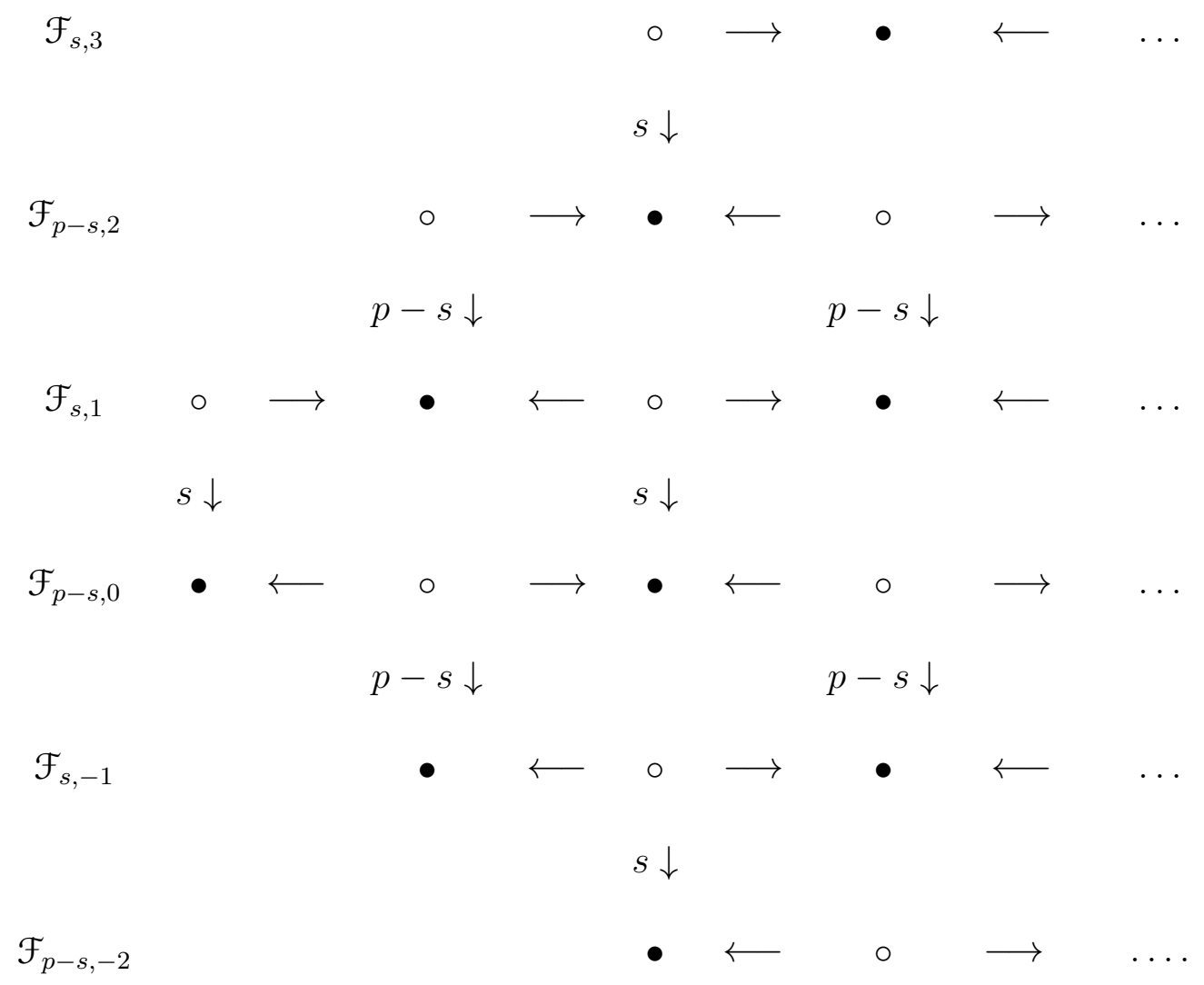

FIGURE 1. Felder complex of Feigin-Fuchs modules for a fixed $s$, where a down arrow like ' $s \downarrow$ ' indicates the action of $s^{\text {th }}$ power of the screening $F=\oint \mathrm{e}^{\alpha_{-} \varphi(z)} d z$ that defines a homomorphism from an upper module with the second index being $n$ to the lower one with $n-1$. The $s$-morphisms and $(p-s)$-morphisms are alternate. Filled dots $\bullet$ correspond to irreducible submodules - they constitute the kernel of such homomorphisms. The conformal dimension of $\mathcal{F}_{s, n}$ is $\Delta_{n, p-s}=\Delta_{1-n, s}$.

Acknowledgments. We are grateful to B.L. Feigin, J. Rasmussen, H. Saleur, A.M. Semikhatov for valuable and stimulating discussions. The work of AMG was supported in part by the RFBR grant 10-01-00408, the RFBR-CNRS grant 09-01-93105, and by the "Landau", "Dynasty" and "Science Support" foundations. The work of PVB was supported in part by the RFBR grant 10-01-00408, the RFBR-CNRS grant 09-01-93105 and "Dynasty" foundation. IYuT is grateful to $\mathrm{H}$. Saleur for kind hospitality in IPhT where a part of the work was made. IYuT was supported in part by the RFBR-CNRS grant 09-02-93106 and the RFBR Grant 08-02-01118. 


\section{Appendix A. Feigin-Fuchs modules}

Here, we remind few simple facts about the well-known Feigin-Fuchs modules which can be found in [50]. The Feigin-Fuchs module $\mathcal{F}_{s, n}$ over the Virasoro algebra $\mathcal{V}_{p}$ is the space generated by all polynomials $P(\partial \phi)$ from the vertex-operator $e^{\left(\frac{1-s}{2} \alpha_{-}+\frac{n}{2} \alpha_{+}\right) \phi}$, where $1 \leqslant s \leqslant p, n \in \mathbb{Z}$, and $\alpha_{+}=\sqrt{2 p}$ and $\alpha_{-}=-\sqrt{2 / p}$. The (lowest) conformal dimension in $\mathcal{F}_{s, n}$ is $\Delta_{n, p-s}=\Delta_{1-n, s}$, where $\Delta_{r, s}$ is defined in (1.7). When $s \neq p$, the $\mathcal{F}_{s, n}$ has a chain-type subquotient structure of one of the following patterns:

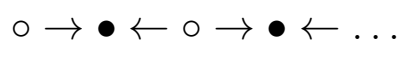

or

$$
\bullet \leftarrow \circ \rightarrow \bullet \leftarrow \circ \rightarrow \ldots
$$

where filled and open dots $\bullet$ and o correspond to irreducible submodules/subquotients.

For a fixed value of $s$, the Feigin-Fuchs modules form a Felder complex [26] (see also [11]) shown in Fig. 1.

\section{ApPendix B. Projective $\mathcal{L} \mathcal{U}_{\mathfrak{q}} s \ell(2)$-MOdules}

Here, we explicitly describe the $\mathcal{L} \mathcal{U}_{\mathfrak{q}} s \ell(2)$ action in the projective module $\mathrm{P}_{s, r}^{ \pm}$. Let $s$ be an integer $1 \leqslant s \leqslant p-1$ and $r \in \mathbb{N}$.

For $r>1$, the projective module $\mathrm{P}_{s, r}^{ \pm}$has the basis

$$
\left\{\mathrm{t}_{n, m}, \mathrm{~b}_{n, m}\right\}_{\substack{0 \leqslant n \leqslant s-1 \\ 0 \leqslant m \leqslant r-1}} \cup\left\{\mathrm{I}_{k, l}\right\}_{\substack{0 \leqslant k \leqslant p-s-1 \\ 0 \leqslant l \leqslant r-2}} \cup\left\{\mathrm{r}_{k, l}\right\}_{\substack{0 \leqslant k \leqslant p-s-1 \\ 0 \leqslant l \leqslant r}},
$$

where $\left\{\mathrm{t}_{n, m}\right\}_{\substack{0 \leqslant n \leqslant s-1 \\ 0 \leqslant m \leqslant r-1}}$ is the basis corresponding to the top module in (1.1), $\left\{\mathrm{b}_{n, m}\right\}_{\substack{0 \leqslant n \leqslant s-1 \\ 0 \leqslant m \leqslant r-1}}$ to the bottom, $\left\{\mathrm{I}_{k, l}\right\}_{\substack{0 \leqslant k \leqslant p-s-1 \\ 0 \leqslant l \leqslant r-2}}$ to the left, and $\left\{\mathrm{r}_{k}\right\}_{\substack{0 \leqslant k \leqslant p-s-1 \\ 0 \leqslant l \leqslant r}}$ to the right module.

For $r=1$, the basis does not contain $\left\{\left.\right|_{k, l}\right\}_{\substack{0 \leqslant k \leqslant p-s-1 \\ 0 \leqslant l \leqslant r-2}}$ terms and we imply $\left.\right|_{k, l} \equiv 0$ in the action. The $\mathcal{L} \mathcal{U}_{\mathfrak{q}} s \ell(2)$-action on $\mathrm{P}_{s, r}^{ \pm}$is given by

$$
\begin{aligned}
& K \mathrm{t}_{n, m}= \pm \mathfrak{q}^{s-1-2 n} \mathrm{t}_{n, m}, \quad 0 \leqslant n \leqslant s-1, \quad 0 \leqslant m \leqslant r-1, \\
& K \mathrm{I}_{k, m}=\left.\mp \mathfrak{q}^{p-s-1-2 k}\right|_{k, m}, \quad 0 \leqslant k \leqslant p-s-1, \quad 0 \leqslant m \leqslant r-2, \\
& K \mathrm{r}_{k, m}=\mp \mathfrak{q}^{p-s-1-2 k} \mathrm{r}_{k, m}, \quad 0 \leqslant k \leqslant p-s-1, \quad 0 \leqslant m \leqslant r, \\
& K \mathrm{~b}_{n, m}= \pm \mathfrak{q}^{s-1-2 n} \mathrm{~b}_{n, m}, \quad 0 \leqslant n \leqslant s-1, \quad 0 \leqslant m \leqslant r-1, \\
& E \mathrm{t}_{n, m}= \begin{cases} \pm[n][s-n] \mathrm{t}_{n-1, m} \pm g \mathrm{~b}_{n-1, m}, \quad 1 \leqslant n \leqslant s-1, \quad 0 \leqslant m \leqslant r-1, \\
\pm g \frac{r-m}{r} \mathrm{r}_{p-s-1, m} \pm\left. g \frac{m}{r}\right|_{p-s-1, m-1}, \quad n=0,\end{cases} \\
& E \mathrm{I}_{k, m}= \begin{cases}\mp[k][p-s-k] \mathrm{I}_{k-1, m}, & 1 \leqslant k \leqslant p-s-1, \\
\pm g(m-r+1) \mathrm{b}_{s-1, m}, & k=0,\end{cases}
\end{aligned}
$$




$$
\begin{aligned}
& E \mathrm{r}_{k, m}=\left\{\begin{array}{ll}
\mp[k][p-s-k] \mathrm{r}_{k-1, m}, & 1 \leqslant k \leqslant p-s-1, \\
\pm g m \mathrm{~b}_{s-1, m-1}, & k=0,
\end{array} \quad 0 \leqslant m \leqslant r,\right. \\
& E \mathrm{~b}_{n, m}= \pm[n][s-n] \mathrm{b}_{n-1, m}, \quad 1 \leqslant n \leqslant s-1, \quad 0 \leqslant m \leqslant r-1 \quad\left(\mathrm{~b}_{-1, m} \equiv 0\right) \text {, } \\
& F \mathrm{t}_{n, m}=\left\{\begin{array}{ll}
\mathrm{t}_{n+1, m}, & 0 \leqslant n \leqslant s-2, \\
\frac{1}{r} \mathrm{r}_{0, m+1}-\frac{1}{r} \mathrm{l}_{0, m}, & n=s-1 \quad\left(\mathrm{I}_{0, r-1} \equiv 0\right),
\end{array} \quad 0 \leqslant m \leqslant r-1,\right. \\
& \left.F\right|_{k, m}=\left\{\begin{array}{ll}
\mathrm{l}_{k+1, m}, & 0 \leqslant k \leqslant p-s-2, \\
\mathrm{~b}_{0, m+1}, & k=p-s-1,
\end{array} \quad 0 \leqslant m \leqslant r-2,\right. \\
& F \mathrm{r}_{k, m}=\left\{\begin{array}{ll}
\mathrm{r}_{k+1, m}, & 0 \leqslant k \leqslant p-s-2, \\
\mathrm{~b}_{0, m}, & k=p-s-1 \quad\left(\mathrm{~b}_{0, r} \equiv 0\right),
\end{array} \quad 0 \leqslant m \leqslant r,\right. \\
& F \mathrm{~b}_{n, m}=\mathrm{b}_{n+1, m}, \quad 1 \leqslant n \leqslant s-1, \quad 0 \leqslant m \leqslant r-1 \quad\left(\mathrm{~b}_{s, m} \equiv 0\right) \text {. }
\end{aligned}
$$

where $g=\frac{(-1)^{p}[s]}{[p-1] !}$. In thus introduced basis, the $s \ell(2)$-generators $e, f$ and $h$ act in $\mathrm{P}_{s, r}^{ \pm}$as in the direct $\operatorname{sum} \mathrm{X}_{s, r}^{ \pm} \oplus \mathrm{X}_{p-s, r-1}^{\mp} \oplus \mathrm{X}_{p-s, r+1}^{\mp} \oplus \mathrm{X}_{s, r}^{ \pm}$(see (2.16)-(2.18) $)$, where for $r=1$ we set $\mathrm{X}_{p-s, 0}^{\mp} \equiv 0$.

\section{ApPendix C. Indecomposable $\mathcal{L} \mathcal{U}_{\mathfrak{q}} s \ell(2)$-MOdUles: EXAMPLES}

Here, we explicitly describe the $\mathcal{L} \mathcal{U}_{\mathfrak{q}} s \ell(2)$ action in the modules $\mathrm{M}_{s, r}^{ \pm}(1), \mathrm{W}_{s, r}^{ \pm}(1), \mathrm{N}_{s, r}^{ \pm}(1)$, $\eta_{s, r}^{ \pm}(1)$, and $\mathrm{W}_{s, r}^{ \pm}(2)$.

C.1. Modules with $n=1$. Let $s$ be an integer $1 \leqslant s \leqslant p-1$ and $r \in \mathbb{N}$. We note that there are subquotients $\mathrm{M}_{p-s, r-1}^{\mp}(1)$ and $\boldsymbol{U}_{s, r}^{ \pm}(1)$ and submodules $\mathrm{W}_{p-s, r-1}^{\mp}(1)$ and $\mathrm{N}_{s, r}^{ \pm}(1)$ in the $\mathrm{P}_{s, r}^{ \pm}$ module. We thus use the formulas in App. $\mathbf{B}$ to describe these modules:

- the module $\mathrm{M}_{p-s, r-1}^{\mp}(1)$ has the basis

$$
\left\{\mathrm{t}_{n, m}\right\}_{\substack{0 \leqslant n \leqslant s-1 \\ 0 \leqslant m \leqslant r-1}} \cup\left\{\mathrm{I}_{k, l}\right\}_{\substack{0 \leqslant k \leqslant p-s-1 \\ 0 \leqslant l \leqslant r-2}} \cup\left\{\mathrm{r}_{k, l}\right\}_{\substack{0 \leqslant k \leqslant p-s-1 \\ 0 \leqslant l \leqslant r}}
$$

- the module $\mathrm{W}_{p-s, r-1}^{\mp}(1)$ has the basis

$$
\left\{\mathrm{b}_{n, m}\right\}_{\substack{0 \leqslant n \leqslant s-1 \\ 0 \leqslant m \leqslant r-1}} \cup\left\{\mathrm{I}_{k, l}\right\}_{\substack{0 \leqslant k \leqslant p-s-1 \\ 0 \leqslant l \leqslant r-2}} \cup\left\{\mathrm{r}_{k, l}\right\}_{\substack{0 \leqslant k \leqslant p-s-1 \\ 0 \leqslant l \leqslant r}},
$$

- the module $\mathrm{N}_{s, r}^{ \pm}(1)$ has the basis

$$
\left\{\mathrm{b}_{n, m}\right\}_{\substack{0 \leqslant n \leqslant s-1 \\ 0 \leqslant m \leqslant r-1}} \cup\left\{\mathrm{r}_{k, l}\right\}_{\substack{0 \leqslant k \leqslant p-s-1 \\ 0 \leqslant l \leqslant r}},
$$

- the module $И_{s, r}^{ \pm}(1)$ has the basis

$$
\left\{\mathrm{t}_{n, m}\right\}_{\substack{0 \leqslant n \leqslant s-1 \\ 0 \leqslant m \leqslant r-1}} \cup\left\{\mathrm{r}_{k, l}\right\}_{\substack{0 \leqslant k \leqslant p-s-1 \\ 0 \leqslant l \leqslant r}},
$$

and the algebra action on these modules coincides with the action on the space $\mathrm{P}_{s, r}^{ \pm}$with uninvolved basis vectors set identically to zero. The action on $\mathrm{P}_{s, r}^{ \pm}$is given above after (B.1). 
C.2. A module with $n=2$. The indecomposable module $\mathrm{W}_{s, r}^{ \pm}(2)$ has the basis (C.5)

$$
\left\{\mathrm{b}_{k, l}^{1}\right\}_{\substack{0 \leqslant k \leqslant p-s-1 \\ 0 \leqslant l \leqslant r}} \cup\left\{\mathrm{b}_{k, l}^{2}\right\}_{\substack{0 \leqslant k \leqslant p-s-1 \\ 0 \leqslant l \leqslant r+2}} \cup\left\{\left.\right|_{k, l}\right\}_{\substack{0 \leqslant k \leqslant s-1 \\ 0 \leqslant l \leqslant r-1}} \cup\left\{\mathrm{m}_{k, l}\right\}_{\substack{0 \leqslant k \leqslant s-1 \\ 0 \leqslant l \leqslant r+1}} \cup\left\{\mathrm{r}_{k, l}\right\}_{\substack{0 \leqslant k \leqslant s-1 \\ 0 \leqslant l \leqslant r+3}} .
$$

The $\mathcal{L} \mathcal{U}_{\mathfrak{q}} s \ell(2)$-action on $\mathrm{W}_{s, r}^{ \pm}(2)$ is given by

$$
\begin{aligned}
& K \mathrm{~m}_{k, l}= \pm \mathfrak{q}^{s-1-2 k} \mathrm{t}_{k, l}, \quad 0 \leqslant k \leqslant s-1, \quad 0 \leqslant l \leqslant r+1, \\
& \left.K\right|_{k, l}= \pm\left.\mathfrak{q}^{s-1-2 k}\right|_{k, l}, \quad 0 \leqslant k \leqslant s-1, \quad 0 \leqslant l \leqslant r-1 \text {, } \\
& K \mathrm{r}_{k, l}= \pm \mathfrak{q}^{s-1-2 k} \mathrm{r}_{k, l}, \quad 0 \leqslant k \leqslant s-1, \quad 0 \leqslant l \leqslant r+3 \text {, } \\
& K \mathrm{~b}_{k, l}^{1}=\mp \mathfrak{q}^{p-s-1-2 k} \mathrm{~b}_{k, l}^{1}, \quad 0 \leqslant k \leqslant p-s-1, \quad 0 \leqslant l \leqslant r, \\
& K \mathrm{~b}_{k, l}^{2}=\mp \mathfrak{q}^{p-s-1-2 k} \mathrm{~b}_{k, l}^{2}, \quad 0 \leqslant k \leqslant p-s-1, \quad 0 \leqslant l \leqslant r+2 \text {, } \\
& E \mathrm{~m}_{k, l}=\left\{\begin{array}{ll} 
\pm[k][s-k] \mathrm{m}_{k-1, l}, & 1 \leqslant k \leqslant s-1, \\
\mp g(l-r-2) \mathrm{b}_{p-s-1, l-1}^{1} \mp g l \mathrm{~b}_{p-s-1, l}^{2}, & k=0,
\end{array} \quad 0 \leqslant l \leqslant r+1,\right. \\
& \left.E\right|_{k, l}=\left\{\begin{array}{ll} 
\pm[k][s-k] \mathrm{I}_{k-1, l}, & 1 \leqslant k \leqslant s-1, \\
\mp g(l-r) \mathrm{b}_{p-s-1, l}^{1}, & k=0,
\end{array} \quad 0 \leqslant l \leqslant r-1,\right. \\
& E \mathrm{r}_{k, l}=\left\{\begin{array}{ll} 
\pm[k][s-k] \mathrm{r}_{k-1, l}, & 1 \leqslant k \leqslant s-1, \\
\mp g l \mathrm{~b}_{p-s-1, l-1}^{2}, & k=0,
\end{array} \quad 0 \leqslant l \leqslant r+3,\right. \\
& E \mathrm{~b}_{k, l}^{1}=\mp[k][p-s-k] \mathrm{b}_{k-1, l}^{1}, \quad 0 \leqslant k \leqslant p-s-1, \quad 0 \leqslant l \leqslant r \quad\left(\mathrm{~b}_{-1, l}^{1} \equiv 0\right) \text {, } \\
& E \mathrm{~b}_{k, l}^{2}=\mp[k][p-s-k] \mathrm{b}_{k-1, l}^{2}, \quad 0 \leqslant k \leqslant p-s-1, \quad 0 \leqslant l \leqslant r+2 \quad\left(\mathrm{~b}_{-1, l}^{2} \equiv 0\right) \text {, } \\
& F \mathrm{~m}_{k, l}=\left\{\begin{array}{ll}
\mathrm{m}_{k+1, l}, & 0 \leqslant k \leqslant s-2, \\
\mathrm{~b}_{0, l}^{1}+\mathrm{b}_{0, l+1}^{2}, & k=s-1 \quad\left(\mathrm{~b}_{0, r+1}^{1} \equiv 0\right),
\end{array} \quad 0 \leqslant l \leqslant r+1,\right. \\
& F \mathrm{I}_{k, l}=\left\{\begin{array}{ll}
\mathrm{l}_{k+1, l}, & 0 \leqslant k \leqslant s-2, \\
\mathrm{~b}_{0, l+1}^{1}, & k=s-1,
\end{array} \quad 0 \leqslant l \leqslant r-1,\right. \\
& F r_{k, l}=\left\{\begin{array}{ll}
\mathrm{r}_{k+1, l}, & 0 \leqslant k \leqslant s-2, \\
\mathrm{~b}_{0, l}^{2}, & k=s-1 \quad\left(\mathrm{~b}_{0, r+3}^{2} \equiv 0\right),
\end{array} \quad 0 \leqslant l \leqslant r+3,\right. \\
& F \mathrm{~b}_{k, l}^{1}=\mathrm{b}_{k+1, l}^{1}, \quad 0 \leqslant k \leqslant p-s-1, \quad 0 \leqslant l \leqslant r \quad\left(\mathrm{~b}_{p-s, l}^{1} \equiv 0\right), \\
& F \mathrm{~b}_{k, l}^{2}=\mathrm{b}_{k+1, l}^{2}, \quad 0 \leqslant k \leqslant p-s-1, \quad 0 \leqslant l \leqslant r+2 \quad\left(\mathrm{~b}_{p-s, l}^{2} \equiv 0\right) \text {. }
\end{aligned}
$$

where $g=\frac{(-1)^{p}[s]}{[p-1] !}$. In thus introduced basis, the $s \ell(2)$-generators $e, f$ and $h$ act in $\mathbf{W}_{s, r}^{ \pm}(2)$ as in the direct sum $\mathrm{X}_{s, r}^{ \pm} \oplus \mathrm{X}_{s, r+2}^{ \pm} \oplus \mathrm{X}_{s, r+4}^{ \pm} \oplus \mathrm{X}_{p-s, r+1}^{\mp} \oplus \mathrm{X}_{p-s, r+3}^{\mp}$, see (2.16)-(2.18).

\section{APPEndix D. Projective RESOlution AND Higher EXTENSION GROUPS}

Here, we construct projective resolutions for irreducible modules. These resolutions involve the modules $\mathrm{W}_{s, r}^{ \pm}$and $\mathrm{N}_{s, r}^{ \pm}$introduced in 3.2. Inspection based on the definition of the 
projective covers in 2.4 with their subquotient structure (1.1), and the definition of indecomposable modules from 3.2 shows that the mappings defined in the following lemma give rise to a projective resolution.

D.1. Proposition. For each $1 \leqslant s \leqslant p-1$ and $\alpha= \pm$,

- the module $\mathrm{X}_{s, 1}^{\alpha}$ has the projective resolution

$$
\ldots \stackrel{\partial_{4}}{\rightarrow} \mathrm{P}_{p-s, 4}^{-\alpha} \stackrel{\partial_{3}}{\rightarrow} \mathrm{P}_{s, 3}^{\alpha} \stackrel{\partial_{2}}{\rightarrow} \mathrm{P}_{p-s, 2}^{-\alpha} \stackrel{\partial_{1}}{\rightarrow} \mathrm{P}_{s, 1}^{\alpha} \stackrel{\partial_{0}}{\rightarrow} \mathrm{X}_{s, 1}^{\alpha}
$$

where for even $n$ the boundary morphism is given by the throughout mapping

$$
\partial_{n}: \mathrm{P}_{s, n+1}^{\alpha} \rightarrow \mathrm{N}_{p-s, n}^{-\alpha}(1) \longmapsto \mathrm{P}_{p-s, n}^{-\alpha},
$$

and for odd $n$, the nth term and the boundary morphism $\partial_{n}$ are given by changing $\alpha$ to $-\alpha$ and $s$ to $p-s$;

- for even $r \geqslant 2$ the module $\mathrm{X}_{s, r}^{\alpha}$ has the projective resolution

$$
\begin{aligned}
& \ldots \stackrel{\partial_{r+2}}{\longrightarrow} \mathrm{P}_{p-s, 3}^{-\alpha} \oplus \mathrm{P}_{p-s, 5}^{-\alpha} \oplus \cdots \oplus \mathrm{P}_{p-s, 2 r+1}^{-\alpha} \stackrel{\partial_{r+1}}{\longrightarrow} \mathrm{P}_{s, 2}^{\alpha} \oplus \cdots \oplus \mathrm{P}_{s, 2 r}^{\alpha} \stackrel{\partial_{r}}{\longrightarrow} \\
& \stackrel{\partial_{r}}{\longrightarrow} \mathrm{P}_{p-s, 1}^{-\alpha} \oplus \mathrm{P}_{p-s, 3}^{-\alpha} \oplus \cdots \oplus \mathrm{P}_{p-s, 2 r-1}^{-\alpha} \stackrel{\partial_{r-1}}{\longrightarrow} \mathrm{P}_{s, 2}^{\alpha} \oplus \mathrm{P}_{s, 4}^{\alpha} \oplus \cdots \oplus \mathrm{P}_{s, 2 r-2}^{\alpha} \stackrel{\partial_{r-2}}{\rightarrow} \\
& \quad \stackrel{\partial_{r-2}}{\longrightarrow} \ldots \stackrel{\partial_{3}}{\rightarrow} \mathrm{P}_{s, r-2}^{\alpha} \oplus \mathrm{P}_{s, r}^{\alpha} \oplus \mathrm{P}_{s, r+2}^{\alpha} \stackrel{\partial_{2}}{\rightarrow} \mathrm{P}_{p-s, r-1}^{-\alpha} \oplus \mathrm{P}_{p-s, r+1}^{-\alpha} \stackrel{\partial_{1}}{\rightarrow} \mathrm{P}_{s, r}^{\alpha} \stackrel{\partial_{0}}{\rightarrow} \mathrm{X}_{s, r}^{\alpha}
\end{aligned}
$$

which consists of two parts separated by the homomorphism $\partial_{r}$ :

* on the right from $\partial_{r}$, i.e. $n<r$, the $n$th term with even $n$ is given by

$$
\stackrel{\partial_{n+1}}{\longrightarrow} \underbrace{\mathrm{P}_{s, r-n}^{\alpha} \oplus \mathrm{P}_{s, r-n+2}^{\alpha} \oplus \cdots \oplus \mathrm{P}_{s, r+n}^{\alpha}}_{n+1} \stackrel{\partial_{n}}{\longrightarrow}
$$

with the boundary morphism given by the throughout mapping in

$$
\partial_{n}: \underbrace{\mathrm{P}_{s, r-n}^{\alpha} \oplus \cdots \oplus \mathrm{P}_{s, r+n}^{\alpha}}_{n+1} \rightarrow \mathrm{W}_{s, r-n}^{\alpha}(n) \longmapsto \underbrace{\mathrm{P}_{p-s, r-n+1}^{-\alpha} \oplus \mathrm{P}_{p-s, r-n+3}^{-\alpha} \oplus \cdots \oplus \mathrm{P}_{p-s, r+n-1}^{-\alpha}}_{n},
$$

and for odd $n$, the nth term and the boundary morphism $\partial_{n}$ are given by changing $\alpha$ to $-\alpha$ and $s$ to $p-s$;

* on the left from $\partial_{r}$ in (D.2), i.e. $n \geqslant r$, the $(r+k)$ th term with even $k \geqslant 0$ is

$$
\stackrel{\partial_{r+k+1}}{\longrightarrow} \underbrace{\mathrm{P}_{s, k+2}^{\alpha} \oplus \mathrm{P}_{s, k+4}^{\alpha} \oplus \cdots \oplus \mathrm{P}_{s, k+2 r}^{\alpha}}_{r} \stackrel{\partial_{r+k}}{\longrightarrow}
$$

with the boundary morphism given by the throughout mapping in

$$
\partial_{r+k}: \underbrace{\mathrm{P}_{s, k+2}^{\alpha} \oplus \cdots \oplus \mathrm{P}_{s, k+2 r}^{\alpha}}_{r} \rightarrow \mathrm{N}_{p-s, k+1}^{-\alpha}(r) \longmapsto \underbrace{\mathrm{P}_{p-s, k+1}^{-\alpha} \oplus \mathrm{P}_{p-s, k+3}^{-\alpha} \oplus \cdots \oplus \mathrm{P}_{p-s, 2 r+k-1}^{-\alpha}}_{r},
$$

and for odd $k$, the $(r+k)$ th term and the boundary morphism $\partial_{r+k}$ are given by changing $\alpha$ to $-\alpha$ and $s$ to $p-s$; 
- for odd $r \geqslant 3$ the module $\mathrm{X}_{s, r}^{\alpha}$ has the projective resolution as in (D.2) with the substitution $\alpha \rightarrow-\alpha$ and $s \rightarrow p-s$ in all terms and morphisms on the left from $\partial_{r-2}$.

D.1.1. Remark. One can easily obtain injectve resolutions dualising the statement in D.1by reversing all the homomorphisms and replacing all the modules of $\mathrm{W}$ - and N-types by corresponding conrtagredient ones, $\mathrm{M}$ - and $\boldsymbol{U}$-modules.

D.2. Higher extensions via the resolution. We now use the projective resolutions to calculate $\operatorname{Ext}_{\mathcal{L} \mathcal{U}_{q}}^{n}$ between simple modules. They are collected in 3.1.1.

The contravariant functor $\operatorname{Hom}_{\mathcal{L} U_{q}}\left(-, \mathrm{X}_{s^{\prime}, r^{\prime}}^{\alpha^{\prime}}\right)$ applied to the projective resolution (D.1) of $\mathrm{X}_{s, 1}^{\alpha}$ described in D.1 gives the cochain complex

$$
0 \stackrel{\delta_{0}}{\rightarrow} \operatorname{Hom}_{\mathcal{L u}_{q}}\left(\mathrm{P}_{s, 1}^{\alpha}, \mathrm{X}_{s^{\prime}, r^{\prime}}^{\alpha^{\prime}}\right) \stackrel{\delta_{1}}{\rightarrow} \operatorname{Hom}_{\mathcal{L} u_{q}}\left(\mathrm{P}_{p-s, 2}^{-\alpha}, \mathrm{X}_{s^{\prime}, r^{\prime}}^{\alpha^{\prime}}\right) \stackrel{\delta_{2}}{\rightarrow} \operatorname{Hom}_{\mathcal{L} \mathcal{U}_{q}}\left(\mathrm{P}_{s, 3}^{\alpha}, \mathrm{X}_{s^{\prime}, r^{\prime}}^{\alpha^{\prime}} \stackrel{\delta_{3}}{\rightarrow} \ldots,\right.
$$

where an odd $n$th term is non-trivial only if $\alpha^{\prime}=\alpha, s^{\prime}=s$ and $r^{\prime}=n$, and it is onedimensional, while an even $n$th term is non-trivial only if $\alpha^{\prime}=-\alpha, s^{\prime}=p-s$ and $r^{\prime}=n$, and it is also one-dimensional. We thus have that all coboundary morphisms $\delta_{i}=0, i \geqslant 0$. The cohomologies $\operatorname{ker}\left(\delta_{n+1}\right) / \operatorname{im}\left(\delta_{n}\right)$ of this complex give then $\operatorname{Ext}_{\mathcal{L} \mathcal{U}_{q}}^{n}\left(\mathrm{X}_{s, 1}^{\alpha}, \mathrm{X}_{s^{\prime}, r^{\prime}}^{\alpha^{\prime}}\right)$.

Applying the contravariant functor $\operatorname{Hom}_{\mathcal{L U}_{q}}\left(-, \mathrm{X}_{s^{\prime}, r^{\prime}}^{\alpha^{\prime}}\right)$ to the projective resolution (D.2) of $\mathrm{X}_{s, r}^{\alpha}$, for even $r$, gives the cochain complex

$$
\begin{aligned}
\mathbf{P}: 0 \stackrel{\delta_{0}}{\rightarrow} \operatorname{Hom}\left(\mathrm{P}_{s, r}^{\alpha}, \mathrm{X}_{s^{\prime}, r^{\prime}}^{\alpha^{\prime}}\right) & \stackrel{\delta_{1}}{\rightarrow} \operatorname{Hom}\left(\mathrm{P}_{p-s, r-1}^{-\alpha} \oplus \mathrm{P}_{p-s, r+1}^{-\alpha}, \mathrm{X}_{s^{\prime}, r^{\prime}}^{\alpha^{\prime}}\right) \stackrel{\delta_{2}}{\rightarrow} \ldots \stackrel{\delta_{r-1}}{\longrightarrow} \\
\stackrel{\delta_{r-1}}{\longrightarrow} \operatorname{Hom}\left(\mathrm{P}_{p-s, 1}^{-\alpha} \oplus\right. & \left.\cdots \oplus \mathrm{P}_{p-s, 2 r-1}^{-\alpha}, \mathrm{X}_{s^{\prime}, r^{\prime}}^{\alpha^{\prime}}\right) \stackrel{\delta_{r}}{\rightarrow} \operatorname{Hom}\left(\mathrm{P}_{s, 2}^{\alpha} \oplus \cdots \oplus \mathrm{P}_{s, 2 r}^{\alpha}, \mathrm{X}_{s^{\prime}, r^{\prime}}^{\alpha^{\prime}}\right) \\
& \stackrel{\delta_{r+1}}{\longrightarrow} \operatorname{Hom}\left(\mathrm{P}_{p-s, 3}^{-\alpha} \oplus \mathrm{P}_{p-s, 5}^{-\alpha} \oplus \cdots \oplus \mathrm{P}_{p-s, 2 r+1}^{-\alpha}, \mathrm{X}_{s^{\prime}, r^{\prime}}^{\alpha^{\prime}}\right) \stackrel{\partial_{r+2}}{\longrightarrow} \ldots
\end{aligned}
$$

where we set Hom $\equiv \operatorname{Hom}_{\mathcal{L u}_{q}}$ and the $(n+1)$ th term for even $n<r$ is given by

$$
\ldots \stackrel{\delta_{n}}{\rightarrow} \operatorname{Hom}_{\mathcal{L} U_{q}}(\underbrace{\mathrm{P}_{s, r-n}^{\alpha} \oplus \mathrm{P}_{s, r-n+2}^{\alpha} \oplus \cdots \oplus \mathrm{P}_{s, r+n}^{\alpha}}_{n+1}, \mathrm{X}_{s^{\prime}, r^{\prime}}^{\alpha^{\prime}}) \stackrel{\delta_{n+1}}{\longrightarrow} \ldots
$$

and for odd $n<r$, the $(n+1)$ th term is

$$
\cdots \stackrel{\delta_{n}}{\rightarrow} \operatorname{Hom}_{\mathcal{L} \mathcal{U}_{q}}(\underbrace{\mathrm{P}_{p-s, r-n}^{-\alpha} \oplus \mathrm{P}_{p-s, r-n+2}^{-\alpha} \oplus \cdots \oplus \mathrm{P}_{p-s, r+n}^{-\alpha}}_{n+1}, \mathrm{X}_{s^{\prime}, r^{\prime}}^{\alpha^{\prime}}) \stackrel{\delta_{n+1}}{\longrightarrow} \ldots
$$

The term in ( $(\overline{\mathrm{D} .3})$ is non-zero only if $s^{\prime}=s, \alpha^{\prime}=\alpha$ and $r^{\prime}=r+2 k$, with $-n / 2 \leqslant k \leqslant n / 2$, and is isomorphic to $\mathbb{C}$ while the term in (D.4) is non-zero when $s^{\prime}=p-s, \alpha^{\prime}=-\alpha$ and $r^{\prime}=r+2 k+1$, with $-(n+1) / 2 \leqslant k \leqslant(n-1) / 2$, and is also isomorphic to $\mathbb{C}$. The two cases have zero intersection and we thus have that all coboundary morphisms $\delta_{i}=0, i \geqslant 1$. The cohomologies $\operatorname{ker}\left(\delta_{n+1}\right) / \operatorname{im}\left(\delta_{n}\right)$ of the complex $\mathbf{P}$ give then the $n$-extension groups $\operatorname{Ext}_{\mathcal{L} U_{q}}^{n}\left(\mathrm{X}_{s, r}^{\alpha}, \mathrm{X}_{s^{\prime}, r^{\prime}}^{\alpha^{\prime}}\right)$ for all $n<r$. The higher-extension groups are calculated using similar analysis of the cohomologies of $\mathbf{P}$ for $n \geqslant r$. 
To calculate $\operatorname{Ext}_{\mathcal{L} \mathcal{U}_{q}}^{n}\left(\mathrm{X}_{s, r}^{\alpha}, \mathrm{X}_{s^{\prime}, r^{\prime}}^{\alpha^{\prime}}\right)$ for odd $r$, we proceed similarly using the projective resolution for $\mathrm{X}_{s, r}^{\alpha}$ in the odd- $r$ case described in D.1.

We note that the $n$-extensions between all irreducible modules computed using the projective resolutions are in remarkable coincidence with the result (3.1) obtained by the direct calculation which uses the spectral sequence.

\section{APPENDIX E. TENSOR PRODUCTS OF INDECOMPOSABLE MODULES}

We use here Thm. 4.3 and (4.30)-(4.31) to give an exhaustive list of tensor products of all indecomposable modules with the semisimple length not greater than 2 .

$$
\begin{aligned}
& \mathbf{X}_{s_{1}, r_{1}}^{\alpha} \otimes \mathbf{N}_{s_{2}, r_{2}}^{\beta}(n)=\left(\mathbf{X}_{s_{1}, r_{1}}^{\alpha} \otimes \mathbf{X}_{1, n}^{\beta}\right) \otimes \mathbf{N}_{s_{2}, r_{2}+n-1}^{+}(1) \\
& \mathbf{X}_{s_{1}, r_{1}}^{\alpha} \otimes \boldsymbol{U}_{s_{2}, r_{2}}^{\beta}(n)=\left(\mathbf{X}_{s_{1}, r_{1}}^{\alpha} \otimes \mathbf{X}_{1, n}^{\beta}\right) \otimes \boldsymbol{U}_{s_{2}, r_{2}+n-1}^{+}(1), \\
& \mathbf{X}_{s_{1}, r_{1}}^{\alpha} \otimes \mathbf{W}_{s_{2}, r_{2}}^{\beta}(n)=\left(\mathbf{X}_{s_{1}, r_{1}}^{\alpha} \otimes \mathbf{X}_{1, r_{2}+n}^{\beta}\right) \otimes \mathbf{N}_{p-s_{2}, n}^{-}(1), \\
& \mathrm{X}_{s_{1}, r_{1}}^{\alpha} \otimes \mathrm{M}_{s_{2}, r_{2}}^{\beta}(n)=\left(\mathrm{X}_{s_{1}, r_{1}}^{\alpha} \otimes \mathrm{X}_{1, r_{2}+n}^{\beta}\right) \otimes \boldsymbol{U}_{p-s_{2}, n}^{-}(1), \\
& \mathrm{N}_{s_{1}, r_{1}}^{\alpha}\left(n_{1}\right) \otimes \mathbf{N}_{s_{2}, r_{2}}^{\beta}\left(n_{2}\right)=\left(\mathbf{X}_{1, n_{1}}^{\alpha} \otimes \mathbf{X}_{1, n_{2}}^{\beta}\right) \otimes\left(\mathbf{N}_{s_{1}, r_{1}+n_{1}-1}^{+}(1) \otimes \mathbf{N}_{s_{2}, r_{2}+n_{2}-1}^{+}(1)\right), \\
& \mathrm{N}_{s_{1}, r_{1}}^{\alpha}\left(n_{1}\right) \otimes \boldsymbol{U}_{s_{2}, r_{2}}^{\beta}\left(n_{2}\right)=\left(\mathbf{X}_{1, n_{1}}^{\alpha} \otimes \mathbf{X}_{1, n_{2}}^{\beta}\right) \otimes\left(\mathbf{N}_{s_{1}, r_{1}+n_{1}-1}^{+}(1) \otimes \boldsymbol{И}_{s_{2}, r_{2}+n_{2}-1}^{+}(1)\right), \\
& \mathrm{N}_{s_{1}, r_{1}}^{\alpha}\left(n_{1}\right) \otimes \mathbf{W}_{s_{2}, r_{2}}^{\beta}\left(n_{2}\right)=\left(\mathbf{X}_{1, n_{1}}^{\alpha} \otimes \mathbf{X}_{1, r_{2}+n_{2}}^{\beta}\right) \otimes\left(\mathbf{N}_{s_{1}, r_{1}+n_{1}-1}^{+}(1) \otimes \mathbf{N}_{p-s_{2}, n_{2}}^{-}(1)\right) \text {, } \\
& \mathrm{N}_{s_{1}, r_{1}}^{\alpha}\left(n_{1}\right) \otimes \mathbf{M}_{s_{2}, r_{2}}^{\beta}\left(n_{2}\right)=\left(\mathbf{X}_{1, n_{1}}^{\alpha} \otimes \mathbf{X}_{1, r_{2}+n_{2}}^{\beta}\right) \otimes\left(\mathbf{N}_{s_{1}, r_{1}+n_{1}-1}^{+}(1) \otimes \boldsymbol{U}_{p-s_{2}, n_{2}}^{-}(1)\right), \\
& \boldsymbol{U}_{s_{1}, r_{1}}^{\alpha}\left(n_{1}\right) \otimes \boldsymbol{U}_{s_{2}, r_{2}}^{\beta}\left(n_{2}\right)=\left(\mathrm{X}_{1, n_{1}}^{\alpha} \otimes \mathrm{X}_{1, n_{2}}^{\beta}\right) \otimes\left(\boldsymbol{U}_{s_{1}, r_{1}+n_{1}-1}^{+}(1) \otimes \boldsymbol{И}_{s_{2}, r_{2}+n_{2}-1}^{+}(1)\right) \\
& \boldsymbol{U}_{s_{1}, r_{1}}^{\alpha}\left(n_{1}\right) \otimes \mathbf{W}_{s_{2}, r_{2}}^{\beta}\left(n_{2}\right)=\left(\mathrm{X}_{1, n_{1}}^{\alpha} \otimes \mathrm{X}_{1, r_{2}+n_{2}}^{\beta}\right) \otimes\left(\boldsymbol{U}_{s_{1}, r_{1}+n_{1}-1}^{+}(1) \otimes \mathbf{N}_{p-s_{2}, n_{2}}^{-}(1)\right) \text {, } \\
& \boldsymbol{U}_{s_{1}, r_{1}}^{\alpha}\left(n_{1}\right) \otimes \mathrm{M}_{s_{2}, r_{2}}^{\beta}\left(n_{2}\right)=\left(\mathrm{X}_{1, n_{1}}^{\alpha} \otimes \mathrm{X}_{1, r_{2}+n_{2}}^{\beta}\right) \otimes\left(\boldsymbol{U}_{s_{1}, r_{1}+n_{1}-1}^{+}(1) \otimes \boldsymbol{И}_{p-s_{2}, n_{2}}^{-}(1)\right), \\
& \mathbf{W}_{s_{1}, r_{1}}^{\alpha}\left(n_{1}\right) \otimes \mathbf{W}_{s_{2}, r_{2}}^{\beta}\left(n_{2}\right)=\left(\mathbf{X}_{1, r_{1}+n_{1}}^{\alpha} \otimes \mathbf{X}_{1, r_{2}+n_{2}}^{\beta}\right) \otimes\left(\mathbf{N}_{p-s_{1}, n_{1}}^{-}(1) \otimes \mathbf{N}_{p-s_{2}, n_{2}}^{-}(1)\right) \text {, } \\
& \mathrm{W}_{s_{1}, r_{1}}^{\alpha}\left(n_{1}\right) \otimes \mathrm{M}_{s_{2}, r_{2}}^{\beta}\left(n_{2}\right)=\left(\mathrm{X}_{1, r_{1}+n_{1}}^{\alpha} \otimes \mathrm{X}_{1, r_{2}+n_{2}}^{\beta}\right) \otimes\left(\mathbf{N}_{p-s_{1}, n_{1}}^{-}(1) \otimes \boldsymbol{И}_{p-s_{2}, n_{2}}^{-}(1)\right), \\
& \mathrm{M}_{s_{1}, r_{1}}^{\alpha}\left(n_{1}\right) \otimes \mathrm{M}_{s_{2}, r_{2}}^{\beta}\left(n_{2}\right)=\left(\mathrm{X}_{1, r_{1}+n_{1}}^{\alpha} \otimes \mathrm{X}_{1, r_{2}+n_{2}}^{\beta}\right) \otimes\left(\boldsymbol{U}_{p-s_{1}, n_{1}}^{-}(1) \otimes \boldsymbol{И}_{p-s_{2}, n_{2}}^{-}(1)\right) .
\end{aligned}
$$

Explicit decompositions of these tensor products easily follow by applying (4.1) and Thm.4.1, and Thm. 4.2.

\section{APPEndix F. THE $A_{N}$ QUIVERS}

We here recall basic notions about quivers [54, 55]. 
F.1. Quivers and their representations. A quiver is an oriented graph, that is, a quadruple $(I, A, s, t)$, consisting of a finite set $I$ of vertices, a finite set $A$ of oriented edges (arrows), and two maps $s$ and $t$ from $A$ to $I$. An oriented edge $a \in A$ starts at the vertex $s(a)$ and terminates at $t(a)$.

A representation of a quiver $Q$ (over $\mathbb{C}$ ) is a collection of finite-dimensional vector spaces $V_{i}$ over $\mathbb{C}$, one for each vertex $i \in I$ of $Q$, and $\mathbb{C}$-linear maps $f_{i j}: V_{i} \rightarrow V_{j}$, one for each oriented edge $\stackrel{i}{\bullet} \stackrel{a}{\rightarrow} \bullet$. The dimension of a representation $\rho$ of $Q$ is an element of $\mathbb{Z}[I]$ given by the dimensions of $V_{i}, i \in I: \operatorname{dim}(\rho)=\sum_{i \in I} \operatorname{dim}_{\mathbb{C}}\left(V_{i}\right) i$.

A morphism from a representation $\rho$ of a quiver $Q$ to another representation $\rho^{\prime}$ of $Q$ is an $I$-graded $\mathbb{C}$-linear map $\phi=\bigoplus_{i \in I} \phi_{i}: \bigoplus_{i \in I} V_{i} \rightarrow \bigoplus_{i \in I} V_{i}^{\prime}$ satisfying $f_{i j}^{\prime} \phi_{i}=\phi_{j} f_{i j}$ for each oriented edge $\stackrel{i}{\bullet} \stackrel{a}{\rightarrow} \bullet$. This gives the category of representations of the quiver $Q$, to be denoted by $\operatorname{Rep}(Q)$ in what follows.

If a quiver $Q$ has no oriented cycles, isomorphism classes of simple objects in $\operatorname{Rep}(Q)$ are in a one-to-one correspondence with vertices of $Q$. The simple object corresponding to a vertex $i \in I$ is given by the vector spaces

$$
V_{j}= \begin{cases}\mathbb{C}, & j=i, \\ 0, & \text { otherwise }\end{cases}
$$

and $\mathbb{C}$-linear maps

$$
f_{i j}=0, \quad \text { for all } i, j \in I .
$$

A quiver is said to be of finite type if the underlying nonoriented graph is a Dynkin graph of finite type. A quiver is said to be of simply laced type if it does not have a pair of vertices connected by more than one arrow.

F.2. The category $\operatorname{Rep}(\mathbf{A})$ of representations of the $A_{N}$ quiver. The $A_{N}$ quiver $\mathbf{A}_{N}$ is simply-laced and has $N$ vertices connected by $N-1$ single edges, that is, $\mathbf{A}_{N}=(\{1,2, \ldots, N\}$, $\left.\left\{g_{i}\right\}, s, t\right)$, where $s\left(g_{i}\right)=i+1$ and $t\left(g_{i}\right)=i$ for odd $i$, and $s\left(g_{i}\right)=i$ and $t\left(g_{i}\right)=i+1$ for even $i$ :

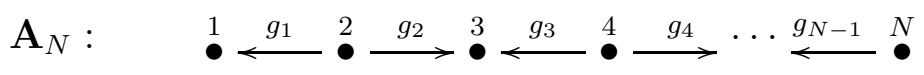

where we also assume that $N$ is even, for simplicity. A representation $\rho$ of $\mathbf{A}_{N}$ is a collection $\left(\left(V_{1}, V_{2}, \ldots, V_{N}\right),\left(f_{2,1}, f_{2,3}, f_{4,3}, f_{4,5}, \ldots, f_{N, N-1}\right)\right)$ consisting of $N$ vector spaces $V_{j}$ and $N-1$ linear maps $f_{j, j \pm 1} \in \operatorname{Hom}_{\mathbb{C}}\left(V_{j}, V_{j \pm 1}\right)$, where $2 \leqslant j \leqslant N$ is even. The dimension of $\rho$ is given by $\operatorname{dim}(\rho)=\left(\operatorname{dim}_{\mathbb{C}}\left(V_{1}\right), \operatorname{dim}_{\mathbb{C}}\left(V_{2}\right), \ldots, \operatorname{dim}_{\mathbb{C}}\left(V_{N}\right)\right)$. Simple objects in the category $\operatorname{Rep}\left(\mathbf{A}_{N}\right)$ are given by the $N$ representations $\rho_{r}=\left(\left(\delta_{i r} \mathbb{C}\right),(0, \ldots, 0)\right)$. We now recall the classification of indecomposable representations of the $A_{N}$ quiver $\mathbf{A}_{N}$, summarized in F.2.1 below. 
There is a correspondence between indecomposable representations of a quiver and the set $\Delta_{+}$of positive roots of the Lie algebra corresponding to the Dynkin graph associated with the quiver. This correspondence is one-to-one for a quiver of simply laced finite type [56, 57]. Namely, a representation $\rho$ of a quiver $Q$ is indecomposable if and only if $\operatorname{dim}(\rho) \in \Delta_{+}$ and, conversely, for every $\alpha \in \Delta_{+}$, there is, up to an isomorphism, a unique indecomposable representation $\rho$ of the quiver $Q$ such that $\operatorname{dim}(\rho)=\alpha$.

The nonoriented graph associated with the quiver $\mathbf{A}_{N}$ is the finite Dynkin graph $A_{N}$. It is well known that $\alpha \in \Delta_{+}$is a positive root of $A_{N}$ if $\alpha=\left(\alpha_{i}\right)$ with $\alpha_{i} \in\{0,1\}$ and at least one $\alpha_{i}=1$ for $1 \leqslant i \leqslant N$. In particular, $\alpha_{r}=\left(\delta_{i r}\right)$, for $1 \leqslant r \leqslant N$, are the simple roots. The simple roots $\alpha_{r}$ correspond to the respective simple objects $\rho_{r}$ in the category $\operatorname{Rep}\left(\mathbf{A}_{N}\right)$. The other positive roots $\alpha$ are in a one-to-one correspondence with indecomposable representations of dimension $\alpha: \rho(\alpha)=\left(\left(\alpha_{i} \mathbb{C}\right),\left(f_{2,1}, f_{2,3}, f_{4,3} \ldots\right)\right)$ with maps $f_{i, i \pm 1}$ defined in an obvious way. We summarize these results in the following well-known proposition (see, e.g., [58]).

\section{F.2.1. Proposition.}

(1) If $\alpha \notin \Delta_{+}$, then the set of indecomposable representations of $\mathbf{A}_{N}$ with the dimension $\alpha$ is empty.

(2) If $\alpha \in \Delta_{+}$, then an indecomposable representation of $\mathbf{A}_{N}$ with the dimension $\alpha$ is either the representation $\rho(\alpha)=\left(\left(\alpha_{i} \mathbb{C}\right),\left(f_{2,1}, f_{2,3}, f_{4,3} \ldots\right)\right)$ with maps $f_{i, i \pm 1}$ defined in an obvious way, where $\alpha=\left(\alpha_{i}\right)$.

\section{REFERENCES}

[1] V.S. Poghosyan, S.Y. Grigorev, V.B. Priezzhev, P. Ruelle, Logarithmic two-point correlators in the Abelian sandpile model, J. Stat. Mech. 1007:P07025, 2010.

[2] K. Kytölä, SLE local martingales in logarithmic representations, J. Stat. Mech. 0908:P08005, 2009.

[3] J. Dubail, J. L. Jacobsen and H. Saleur, Conformal boundary conditions in the critical $O(n)$ model and dilute loop models, Nucl. Phys. B827:457-502,2010.

[4] P. Mathieu, D. Ridout, From Percolation to Logarithmic Conformal Field Theory, Phys. Lett.B657:120129, 2007.

[5] D. Ridout, On the Percolation BCFT and the Crossing Probability of Watts, Nucl. Phys. B810:503-526, 2009.

[6] P.A. Pearce, J. Rasmussen, J.-B. Zuber, Logarithmic Minimal Models, J.Stat.Mech. 0611 (2006) P017.

[7] N. Read and H. Saleur, Exact spectra of conformal supersymmetric nonlinear sigma models in two dimensions, Nucl. Phys. B613 (2001) 409.

[8] R. Bondesan, J. L. Jacobsen, H. Saleur, Edge states and conformal boundary conditions in super spin chains and super sigma models, arXiv:1101.4361,

[9] M.R. Gaberdiel and H.G. Kausch, A local logarithmic conformal field theory, Nucl. Phys. B538 (1999) 631-658 [hep-th/9807091].

[10] M.R. Gaberdiel and H.G. Kausch, A rational logarithmic conformal field theory, Phys. Lett. B 386 (1996) 131 [hep-th/9606050]. 
[11] J. Fuchs, S. Hwang, A.M. Semikhatov, and I.Yu. Tipunin, Nonsemisimple fusion algebras and the Verlinde formula, Commun. Math. Phys. 247 (2004) 713-742 [hep-th/0306274].

[12] B.L. Feigin, A.M. Gainutdinov, A.M. Semikhatov, and I.Yu. Tipunin, Logarithmic extensions of minimal models: characters and modular transformations, Nucl. Phys. B757 (2006) 303-343, hep-th/0606196.

[13] Y.-Z. Huang, J. Lepowsky, and L. Zhang, Logarithmic tensor category theory for generalized modules for a conformal vertex algebra, I-VI, arXiv:1012.4193, arXiv:1012.4196, arXiv:1012.4197; arXiv:1012.4198, arXiv:1012.4199; arXiv:1012.4202

[14] D. Adamović, A. Milas, On W-algebras associated to $(2, p)$ minimal models and their representations, arXiv:0908.4053

[15] D. Adamović, A. Milas, arXiv:1006.5134; Comm. Math. Phys. 288 (2009) 225; Adv. in Math. 217 (2008) 2664.

[16] B.L. Feigin, A.M. Gainutdinov, A.M. Semikhatov, and I.Yu. Tipunin, Modular group representations and fusion in logarithmic conformal field theories and in the quantum group center, Commun. Math. Phys. 265 (2006) 47-93. [hep-th/0504093].

[17] B.L. Feigin, A.M. Gainutdinov, A.M. Semikhatov, and I.Yu. Tipunin, Kazhdan-Lusztig-dual quantum group for logarithmic extensions of Virasoro minimal models, J. Math. Phys. 48 (2007) 032303, [math.QA/0606506].

[18] A.M. Semikhatov, Factorizable ribbon quantum groups in logarithmic conformal field theories, Theor. Math. Phys.154:433-453, 2008; arXiv:0705.4267

[19] A.M. Gainutdinov and I.Yu. Tipunin, Radford, Drinfeld, and Cardy boundary states in $(1, p)$ logarithmic conformal field models, J. Phys. A: Math. Theor. 42 315207, 2009, arXiv:0711.3430,

[20] M.R. Gaberdiel and I. Runkel, From boundary to bulk in logarithmic CFT, arXiv:0707.0388 [hep-th].

[21] P. A. Pearce, J. Rasmussen, Ph. Ruelle, Grothendieck ring and Verlinde-like formula for the W-extended logarithmic minimal model WLM(1,p), J.Phys.A43:045211, 2010.

[22] A.M. Semikhatov, Toward logarithmic extensions of $\widehat{s l}(2)_{k}$ conformal field models, Theor. Math. Phys. 153:1597-1642, 2007.

[23] M. R. Gaberdiel, I. Runkel, S. Wood, Fusion rules and boundary conditions in the $c=0$ triplet model, arXiv:0905.0916, A modular invariant bulk theory for the c=0 triplet model, J.Phys.A44:015204, 2011.

[24] J. Fuchs, Ch. Schweigert, Hopf algebras and finite tensor categories in conformal field theory, Rev. Union Mat. Argentina 51:43-90,2010, arXiv:1004.3405.

[25] B.L. Feigin, I.Yu. Tipunin, Logarithmic CFTs connected with simple Lie algebras, arXiv:1002.5047.

[26] G. Felder, BRST approach to minimal models, Nucl. Phys. B317 (1989) 215-236.

[27] P.V. Bushlanov, B.L. Feigin, A.M. Gainutdinov, and I.Yu. Tipunin, Lusztig limit of quantum sl(2) at root of unity and fusion of $(1, p)$ Virasoro logarithmic minimal models, Nucl. Phys. B 818 [FS] (2009) 179-195; arXiv:0901.1602

[28] H.G. Kausch, Symplectic Fermions, Nucl. Phys. B583 (2000) 513-541.

[29] B.L. Feigin, A.M. Gainutdinov, A.M. Semikhatov, and I.Yu. Tipunin, Kazhdan-Lusztig correspondence for the representation category of the triplet $W$-algebra in logarithmic CFT, Theor. Math. Phys. 148 (2006) 1210-1235. [math.QA/0512621].

[30] D. Kazhdan and G. Lusztig, Tensor structures arising from affine Lie algebras I-IV, J. Amer. Math. Soc. 6 (1993) 905; J. Amer. Math. Soc. 7 (1994) 383.

[31] A.M. Semikhatov, I.Yu. Tipunin, The Nichols algebra of screenings, arXiv:1101.5810,

[32] H.G. Kausch, Extended conformal algebras generated by a multiplet of primary fields, Phys. Lett. B 259 (1991) 448.

[33] K. Nagatomo and A. Tsuchiya, The triplet vertex operator algebra $W(p)$ and the restricted quantum group at root of unity, arXiv:0902.4607 [math.QA]. 
[34] N. Read, H. Saleur, Enlarged symmetry algebras of spin chains, loop models, and S-matrices, Nucl. Phys. B777:263-315,2007.

[35] N. Read and H. Saleur, Associative-algebraic approach to logarithmic conformal field theories, Nucl. Phys. B 777, 316 (2007) [arXiv:hep-th/0701117].

[36] H. N. V. Temperley and E. H. Lieb, Relations between percolation and colouring problems and other graph theoretical problems associated with regular planar lattices: some exact results for the percolation problem, Proceedings of the Royal Society A 322 (1971), 251-280.

[37] V.F.R. Jones, Quotient of the affine Hecke algebra in the Brauer algebra, L'Ens. Math. 40 (1994) 313.

[38] J.J. Graham and G.I. Lehrer, The representation theory of affine Temperley-Lieb algebras, L'Ens. Math. 44 (1998) 173.

[39] R. Brauer, On algebras which are connected with the semisimple continuous groups, Annals of Mathematics 38 (1937), 854-872.

[40] P. P. Martin, The decomposition matrices of the Brauer algebra over the complex field, arXiv:0908.1500.

[41] P.P. Martin and H. Saleur, The blob algebra and the periodic Temperley-Lieb algebra, Lett. Math. Phys. 30 (1994) 189.

[42] J.J. Graham and G. I. Lehrer, The Two-Step Nilpotent Representations of the Extended Affine Hecke Algebra of Type A, Compositio Mathematica 133 (2002) 173.

[43] P.P. Martin, Potts Models and Related Problems in Statistical Mechanics (World Scientific, Singapore, 1991); P. P. Martin, Int. J. Mod. Phys. A 7, Supp. 1B, 645 (1992).

[44] F. Goodman, P. de la Harpe and V. Jones, Coxeter graphs and towers of algebras, MSRI Publications Number 14, Springer, 1989.

[45] V. Pasquier and H. Saleur, Common structures between finite systems and conformal field theories through quantum groups, Nucl. Phys. B 330, 523 (1990).

[46] W.M. Koo and H. Saleur, Representations of the Virasoro algebra from lattice models, Nucl. Phys. B426 (1994) 459-504.

[47] M.R. Gaberdiel and H.G. Kausch, Indecomposable Fusion Products, Nucl. Phys. B477:293-318, 1996.

[48] J. Rasmussen and P. A. Pearce, Fusion Algebras of Logarithmic Minimal Models, J. Phys. A 40, 13711 (2007) [arXiv:0707.3189 [hep-th]].

[49] J. Rasmussen, Classification of Kac representations in the logarithmic minimal models LM(1,p), arXiv: 1012.5190

[50] B.L. Feigin, D.B. Fuchs, Representations of the Virasoro algebra in Representations of infinitedimentional Lie groups and Lie algebras, Gordon and Breach, New York, 1989.

[51] C. Kassel, Quantum Groups, Springer-Verlag, New York, 1995.

[52] K. Kytölä, D. Ridout, On Staggered Indecomposable Virasoro Modules, J. Math. Phys. 50:123503, 2009.

[53] S. MacLane, Homology, Springer-Verlag, 1963.

[54] W. Crawley-Boevey, Lectures on representations of quivers, Lectures in Oxford in 1992, available at http://www.amsta.leeds.ac.uk/pmtwc/.

[55] M. Auslander, I. Reiten, and S.O. Smalø, Representation Theory of Artin Algebras, Cambridge Studies in Adv. Math. vol. 36, Cambridge Univ. Press, 1995.

[56] P. Gabriel, Unzerlegbare Darstellungen. I, Manuscripta Math. 6 (1972), 71-103; correction, ibid. 6 (1972), 309.

[57] J. Bernstein, I. Gelfand, and V. Ponomarev, Coxeter functors and Gabriel's theorem, Uspekhi Mat. Nauk 28 (1973), no. 2(170), 19-33.

[58] I. Frenkel, A. Malkin, M. Vybornov, Affine Lie algebras and tame quivers, [math.RT/0005119]. 
PVB:Moscow Institute of Physics And Technology, Dolgoprudny, Institutskiy Per. 9 , RUSSIA, 141700

E-mail address: paulbush@mail.ru

AMG:Institut de Physique Théorique, CEA Saclay, Gif Sur Yvette, 91191, France

E-mail address: azat.gaynutdinov@cea.fr

IYuT:Tamm Theory Division, Lebedev Physics Institute, Leninski PR., 53, Moscow, RusSIA, 119991

E-mail address: tipuninegmail.com 